Supporting Information

for

\title{
Pd-Pt Heterobimetallic Complexes with Bridging Si-Ligands. Structure and Reaction with Isonitrile to Afford a Platinacyclopentane Containing Si, N, and C Atoms
}

\author{
Tetsuyuki Yamada, ${ }^{\mathrm{a}}$ Makoto Tanabe, ${ }^{\mathrm{a}}$ Kohtaro Osakada, ${ }^{\mathrm{a} *}$ and Yong-Joo Kim ${ }^{\mathrm{b}}$ \\ a Chemical Resources Laboratory, Tokyo Institute of Technology, 4259 Nagatsuta, \\ Midori-ku, Yokohama 226-8503, Japan \\ and ${ }^{b}$ Department of Chemistry Kangnung National University, Kangnung 210-702, Korea
}

$\begin{array}{ll}\text { Crystallographic Results of complex } \mathbf{1} & \text { S1 } \\ \text { Crystallographic Results of complex } 2 & \text { S8 } \\ \text { Crystallographic Results of complex } 3 & \text { S28 } \\ \text { Crystallographic Results of complex } 4 & \text { S45 }\end{array}$ 


\title{
Crystallographic Results of complex 1
}

\section{Data Collection}

\author{
Experimental
}

A colorless prism crystal of $\mathrm{C}_{36} \mathrm{H}_{52} \mathrm{Si}_{2} \mathrm{P}_{2} \mathrm{PdPt}$ having approximate dimensions of $0.20 \mathrm{x}$ $0.20 \times 0.20 \mathrm{~mm}$ was mounted on a glass fiber. All measurements were made on a Rigaku Saturn $\mathrm{CCD}$ area detector with graphite monochromated Mo-K・ radiation.

Indexing was performed from 0 images that were exposed for 0 seconds. The crystal-to-detector distance was $44.88 \mathrm{~mm}$.

Cell constants and an orientation matrix for data collection corresponded to a primitive triclinic cell with dimensions:

$$
\begin{array}{lcc}
\mathrm{a}= & 9.6294(7) \AA & \alpha=78.89(3)^{\mathrm{O}} \\
\mathrm{b}= & 10.1484(5) \AA & \beta=68.03(3)^{\mathrm{O}} \\
\mathrm{c}= & 11.3224 \AA & \gamma=64.21(2)^{\mathrm{O}} \\
\mathrm{V}= & 923.27(8) \AA 3 &
\end{array}
$$

For Z $=1$ and F.W. $=904.42$, the calculated density is $1.63 \mathrm{~g} / \mathrm{cm}^{3}$. Based on a statistical analysis of intensity distribution, and the successful solution and refinement of the structure, the space group was determined to be:

$$
\text { P-1 (\#2) }
$$

The data were collected at a temperature of $20 \pm 1^{\circ} \mathrm{C}$ to a maximum $2 \theta$ value of $55.0^{\circ}$. A total of 720 oscillation images were collected. A sweep of data was done using $\omega$ scans from -110.0 to $70.0^{\circ}$ in $0.5^{\circ}$ step, at $\chi=45.0^{\circ}$ and $\phi=0.0^{\circ}$. The exposure rate was $10.0\left[\mathrm{sec} . /{ }^{\circ}\right.$ ]. The detector swing angle was $-20.34^{\circ}$. A second sweep was performed using $\omega$ scans from -110.0 to $70.0^{\circ}$ in $0.5^{\circ}$ step, at $\chi=45.0^{\circ}$ and $\phi=90.0^{\circ}$. The exposure rate was 10.0 [sec. $/ /^{\circ}$ ]. The detector swing angle was $-20.34^{\circ}$. The crystal-to-detector distance was $44.88 \mathrm{~mm}$. Readout was performed in the $0.070 \mathrm{~mm}$ pixel mode.

\section{Data Reduction}

A total of 5206 reflections was collected. Data were collected and processed using CrystalClear (Rigaku). Net intensities and sigmas were derived as follows:

$$
\begin{aligned}
& \mathrm{F}^{2}=\left[\Sigma\left(\mathrm{P}_{\mathrm{i}}-\mathrm{mB}_{\mathrm{ave}}\right)\right] \cdot \mathrm{Lp}^{-1} \\
& \text { where } \mathrm{P}_{\mathrm{i}} \text { is the value in counts of the } \mathrm{ith} \text { pixel } \\
& \mathrm{m} \text { is the number of pixels in the integration area } \\
& \quad \mathrm{B}_{\text {ave }} \text { is the background average } \\
& \mathrm{Lp} \text { is the Lorentz and polarization factor } \\
& \mathrm{B}_{\text {ave }}=\Sigma\left(\mathrm{B}_{\mathrm{j}}\right) / \mathrm{n} \\
& \text { where } \mathrm{n} \text { is the number of pixels in the background area } \\
& \quad \mathrm{Bj}_{\mathrm{j}} \text { is the value of the } \mathrm{j} \text { th pixel in counts } \\
& \sigma^{2}\left(\mathrm{~F}^{2} \mathrm{hkl}\right)=\left[\left(\Sigma \mathrm{P}_{\mathrm{i}}\right)+\mathrm{m}\left(\left(\bullet\left(\mathrm{B}_{\text {ave }}-\mathrm{B}_{\mathrm{j}}\right)^{2}\right) /(\mathrm{n}-1)\right)\right] \cdot \mathrm{Lp} \cdot \text { errmul }+\left(\text { erradd } \cdot \mathrm{F}^{2}\right)^{2} \\
& \text { where erradd }=0.00 \\
& \text { errmul }=1.00
\end{aligned}
$$

The linear absorption coefficient, $\bullet$, for Mo-K $\bullet$ radiation is $44.3 \mathrm{~cm}^{-1}$. The data were corrected for Lorentz and polarization effects. 
$\underline{\text { Structure Solution and Refinement }}$

The structure was solved by direct methods ${ }^{2}$ and expanded using Fourier techniques 3 . The non-hydrogen atoms were refined anisotropically. Hydrogen atoms were refined using the riding model. The final cycle of full-matrix least-squares refinement ${ }^{4}$ on $\mathrm{F}$ was based on 5075 observed reflections $(\mathrm{I}>3.00 \sigma(\mathrm{I})$ ) and 215 variable parameters and converged (largest parameter shift was 0.00 times its esd) with unweighted and weighted agreement factors of:

$$
\begin{gathered}
\mathrm{R}=\Sigma\|\mathrm{Fo}|-| \mathrm{Fc}\| / \Sigma|\mathrm{Fo}|=0.044 \\
\mathrm{R}_{\mathrm{W}}=\left[\Sigma \mathrm{W}(|\mathrm{Fo}|-|\mathrm{Fc}|)^{2} / \Sigma \mathrm{W} \mathrm{Fo}\right]^{1 / 2}=0.071
\end{gathered}
$$

The standard deviation of an observation of unit weight ${ }^{5}$ was 1.13 . A Sheldrick weighting scheme was used. Plots of $\Sigma \mathrm{w}(|\mathrm{Fo}|-|\mathrm{Fc}|)^{2}$ versus $|\mathrm{Fo}|$, reflection order in data collection, $\sin \theta / \lambda$ and various classes of indices showed no unusual trends. The maximum and minimum peaks on the final difference Fourier map corresponded to 2.84 and $-2.19 \mathrm{e}^{-} / \AA^{3}$, respectively.

Neutral atom scattering factors were taken from Cromer and Waber ${ }^{6}$. Anomalous dispersion effects were included in Fcalc 7 ; the values for $\Delta \mathrm{f}^{\prime}$ and $\Delta \mathrm{f}^{\prime \prime}$ were those of Creagh and McAuley 8 . The values for the mass attenuation coefficients are those of Creagh and Hubbell 9 . All calculations were performed using the CrystalStructure 10,11 crystallographic software package.

\section{References}

(1) CrystalClear: Rigaku Corporation, 1999. CrystalClear Software User's Guide, Molecular Structure Corporation, (c) 2000.J.W.Pflugrath (1999) Acta Cryst. D55, 1718-1725.

(2) SHELX97: Sheldrick, G.M. (1997).

(3) DIRDIF99: Beurskens, P.T., Admiraal, G., Beurskens, G., Bosman, W.P., de Gelder, R., Israel, R. and Smits, J.M.M.(1999). The DIRDIF-99 program system, Technical Report of the Crystallography Laboratory, University of Nijmegen, The Netherlands.

(4) Least Squares function minimized:

$$
\Sigma w\left(\left|\mathrm{~F}_{\mathrm{O}}\right|-\left|\mathrm{F}_{\mathrm{C}}\right|\right)^{2} \quad \text { where } \mathrm{w}=\text { Least Squares weights. }
$$

(5) Standard deviation of an observation of unit weight:

$$
\begin{array}{ll}
{\left[\sum w\left(\left|\mathrm{~F}_{\mathrm{O}}\right|-\left|\mathrm{F}_{\mathrm{C}}\right|\right)^{2} /\left(\mathrm{N}_{\mathrm{O}}-\mathrm{N}_{\mathrm{V}}\right)\right]^{1 / 2}} \\
\text { where: } & \mathrm{N}_{\mathrm{O}}=\text { number of observations } \\
& \mathrm{N}_{\mathrm{V}}=\text { number of variables }
\end{array}
$$

(6) Cromer, D. T. \& Waber, J. T.; "International Tables for X-ray Crystallography", Vol. IV, The Kynoch Press, Birmingham, England, Table 2.2 A (1974).

(7) Ibers, J. A. \& Hamilton, W. C.; Acta Crystallogr., 17, 781 (1964).

(8) Creagh, D. C. \& McAuley, W.J .; "International Tables for Crystallography", Vol C, (A.J.C.

Wilson, ed.), Kluwer Academic Publishers, Boston, Table 4.2.6.8, pages 219-222 (1992).

(9) Creagh, D. C. \& Hubbell, J.H..; "International Tables for Crystallography", Vol C, (A.J.C.

Wilson, ed.), Kluwer Academic Publishers, Boston, Table 4.2.4.3, pages 200-206 (1992).

(10) CrystalStructure 3.10: Crystal Structure Analysis Package, Rigaku and Rigaku/MSC (2000-2002).

(11) CRYSTALS Issue 10: Watkin, D.J., Prout, C.K. Carruthers, J.R. \& Betteridge, P.W. Chemical Crystallography Laboratory, Oxford, UK. 
Empirical Formula

Formula Weight

Crystal Color, Habit

Crystal Dimensions

Crystal System

Lattice Type

Detector Position

Pixel Size

Lattice Parameters

Space Group

$\mathrm{Z}$ value

$D_{\text {calc }}$

$\mathrm{F}_{000}$

$\mu(\mathrm{MoK} \bullet)$

Detector

Goniometer

Radiation

Detector Aperture

Data Images

$\omega$ oscillation Range $(\bullet=45.0, \bullet=0.0)$

Exposure Rate

Detector Swing Angle

$\omega$ oscillation Range $(\bullet=45.0, \bullet=90.0)$

Exposure Rate

Detector Swing Angle

Detector Position

Pixel Size

$2 \theta_{\text {max }}$

No. of Reflections Measured

Corrections
A. Crystal Data

$\mathrm{C}_{36} \mathrm{H}_{52} \mathrm{Si}_{2} \mathrm{P}_{2} \mathrm{PdPt}$

904.42

colorless, prism

$0.50 \times 0.50 \times 0.50 \mathrm{~mm}$

triclinic

Primitive

$44.88 \mathrm{~mm}$

$0.035 \mathrm{~mm}$

$\mathrm{a}=9.6294(7) \AA$

$\mathrm{b}=10.1484(5) \AA$

$\mathrm{c}=11.3224 \AA$

$\alpha=78.89(3)^{\mathrm{o}}$

$\beta=68.03(3)^{\mathrm{o}}$

$\gamma=64.21(2)^{\mathrm{o}}$

$\mathrm{V}=923.27(8) \AA^{3}$

P-1 (\#2)

1

$1.627 \mathrm{~g} / \mathrm{cm}^{3}$

450.00

$44.31 \mathrm{~cm}^{-1}$

B. Intensity Measurements

Rigaku Saturn

Rigaku AFC10

$\operatorname{MoK} \bullet(\bullet=0.71070 \AA)$

graphite monochromated

$70 \mathrm{~mm}$ x $70 \mathrm{~mm}$

720 exposures

$-110.0-70.0^{\circ}$

$10.0 \mathrm{sec} . / \mathrm{O}$

$-20.340$

$-110.0-70.0^{\circ}$

$10.0 \mathrm{sec} . / \mathrm{O}$

$-20.34^{\mathrm{O}}$

$44.88 \mathrm{~mm}$

$0.035 \mathrm{~mm}$

$55.0^{\circ}$

Total: 5206

Lorentz-polarization

C. Structure Solution and Refinement 
Structure Solution

Refinement

Function Minimized

Least Squares Weights

Anomalous Dispersion

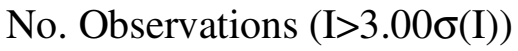

No. Variables

Reflection/Parameter Ratio

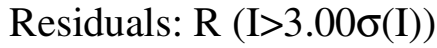

Residuals: Rw (I>3.00б(I))

Goodness of Fit Indicator

Max Shift/Error in Final Cycle

Maximum peak in Final Diff. Map

Minimum peak in Final Diff. Map
Direct Methods (SHELX97)

Full-matrix least-squares on $\mathrm{F}$

$\Sigma \mathrm{w}(|\mathrm{Fo}|-|\mathrm{Fc}|)^{2}$

$1 /\left[0.001 \mathrm{Fo}^{2}+3.000 \sigma^{2}(\mathrm{Fo})+0.500\right]$

All non-hydrogen atoms

5075

215

23.60

0.044

0.071

1.13

0.00

$2.84 \mathrm{e}^{-} / \AA^{3}$

$-2.19 \mathrm{e}^{-} / \AA^{3}$

Table 1. Atomic coordinates and $\mathrm{B}_{\mathrm{iso}} / \mathrm{B}_{\mathrm{eq}}$ and occupancy

$\begin{array}{lclllr}\text { atom } & \mathrm{x} & \mathrm{y} & \mathrm{z} & \mathrm{B} e \mathrm{e} & \mathrm{occ} \\ \mathrm{Pt}(1) & -0.00587(2) & 0.879030(10) & 0.069290(10) & 0.919(7) & 1 / 2 \\ \mathrm{Pd}(1) & -0.00587(2) & 0.879030(10) & 0.069290(10) & 0.919(7) & 1 / 2 \\ \mathrm{P}(1) & -0.00856(13) & 0.71568(10) & 0.23969(8) & 1.30(2) & \\ \mathrm{Si}(1) & 0.02637(12) & 1.06027(10) & 0.13980(9) & 1.00(2) & \\ \mathrm{C}(1) & 0.2254(5) & 1.0119(4) & 0.1635(3) & 1.43(7) & \\ \mathrm{C}(2) & 0.2533(5) & 1.1100(5) & 0.2147(4) & 1.78(8) & \\ \mathrm{C}(3) & 0.4091(5) & 1.0788(5) & 0.2178(4) & 2.05(9) & \\ \mathrm{C}(4) & 0.5379(6) & 0.9490(5) & 0.1692(4) & 2.39(9) & \\ \mathrm{C}(5) & 0.5128(5) & 0.8496(5) & 0.1202(4) & 2.16(8) & \\ \mathrm{C}(6) & 0.3588(5) & 0.8818(4) & 0.1171(4) & 1.86(8) & \\ \mathrm{C}(7) & -0.1436(5) & 1.1636(4) & 0.2825(3) & 1.16(7) & \\ \mathrm{C}(8) & -0.1306(5) & 1.1430(4) & 0.4044(3) & 1.58(7) & \\ \mathrm{C}(9) & -0.2644(5) & 1.2092(5) & 0.5099(4) & 1.91(8) & \\ \mathrm{C}(10) & -0.4121(5) & 1.3033(5) & 0.4952(4) & 1.98(8) & \\ \mathrm{C}(11) & -0.4302(5) & 1.3302(5) & 0.3740(4) & 2.14(8) & \\ \mathrm{C}(12) & -0.2968(4) & 1.2587(4) & 0.2706(3) & 1.40(7) & \\ \mathrm{C}(13) & 0.1932(5) & 0.5853(5) & 0.2434(4) & 2.23(8) & \\ \mathrm{C}(14) & 0.3001(6) & 0.5009(5) & 0.1221(5) & 2.97(10) & \\ \mathrm{C}(15) & -0.1052(5) & 0.7859(5) & 0.4015(4) & 2.05(8) & \\ \mathrm{C}(16) & -0.2848(5) & 0.8879(5) & 0.4288(4) & 2.24(9) & \\ \mathrm{C}(17) & -0.1151(5) & 0.6018(4) & 0.2460(4) & 1.88(8) & \\ \mathrm{C}(18) & -0.1248(9) & 0.4995(6) & 0.3581(6) & 4.7(2) & \\ \mathrm{H}(1) & 0.1654(5) & 1.1994(5) & 0.2472(4) & 2.31(13) & \\ \mathrm{H}(2) & 0.4256(5) & 1.1456(5) & 0.2540(4) & 2.65(13) & \\ \mathrm{H}(3) & 0.6434(6) & 0.9292(5) & 0.1700(4) & 3.0(1) & \\ \mathrm{H}(4) & 0.6015(5) & 0.7602(5) & 0.0887(4) & 2.25(13) & \end{array}$




$\begin{array}{lrlll}\mathrm{H}(5) & 0.3429(5) & 0.8131(4) & 0.0828(4) & 2.11(12) \\ \mathrm{H}(6) & -0.0283(5) & 1.0804(4) & 0.4150(3) & 1.76(12) \\ \mathrm{H}(7) & -0.2530(5) & 1.1903(5) & 0.5920(4) & 2.27(13) \\ \mathrm{H}(8) & -0.5026(5) & 1.3496(5) & 0.5671(4) & 2.16(12) \\ \mathrm{H}(9) & -0.5318(5) & 1.3951(5) & 0.3632(4) & 2.29(13) \\ \mathrm{H}(10) & -0.3094(4) & 1.2766(4) & 0.1890(3) & 1.62(12) \\ \mathrm{H}(11) & 0.1793(5) & 0.5175(5) & 0.3129(4) & 2.44(13) \\ \mathrm{H}(12) & 0.2476(5) & 0.6374(5) & 0.2562(4) & 2.45(13) \\ \mathrm{H}(13) & 0.4117(6) & 0.4761(5) & 0.1095(5) & 2.80(13) \\ \mathrm{H}(14) & 0.2722(6) & 0.5595(5) & 0.0516(5) & 2.77(13) \\ \mathrm{H}(15) & 0.2828(6) & 0.4139(5) & 0.1296(5) & 2.76(13) \\ \mathrm{H}(16) & -0.0963(5) & 0.7052(5) & 0.4611(4) & 2.37(12) \\ \mathrm{H}(17) & -0.0492(5) & 0.8386(5) & 0.4106(4) & 2.37(12) \\ \mathrm{H}(18) & -0.2950(5) & 0.9859(5) & 0.4055(4) & 2.61(13) \\ \mathrm{H}(19) & -0.3302(5) & 0.8598(5) & 0.3814(4) & 2.61(13) \\ \mathrm{H}(20) & -0.3414(5) & 0.8809(5) & 0.5172(4) & 2.61(13) \\ \mathrm{H}(21) & -0.2228(5) & 0.6640(4) & 0.2465(4) & 2.35(13) \\ \mathrm{H}(22) & -0.0583(5) & 0.5452(4) & 0.1719(4) & 2.35(13) \\ \mathrm{H}(23) & -0.0321(9) & 0.4093(6) & 0.3392(6) & 5.9(2) \\ \mathrm{H}(24) & -0.2212(9) & 0.4826(6) & 0.3801(6) & 5.9(2) \\ \mathrm{H}(25) & -0.1280(9) & 0.5418(6) & 0.4275(6) & 5.9(2) \\ & & & & \end{array}$

$\mathrm{B}_{\mathrm{eq}}=8 / 3 \pi^{2}\left(\mathrm{U}_{11}(\mathrm{aa} *)^{2}+\mathrm{U}_{22}\left(\mathrm{bb}^{*}\right)^{2}+\mathrm{U}_{33}(\mathrm{cc} *)^{2}+2 \mathrm{U}_{12}(\mathrm{aa} * \mathrm{bb} *) \cos \gamma+2 \mathrm{U}_{13}(\mathrm{aa} * \mathrm{cc} *) \cos \beta+\right.$ $\left.2 \mathrm{U}_{23}\left(\mathrm{bb}^{*} \mathrm{cc} *\right) \cos \alpha\right)$

Table 2. Anisotropic Displacement Parameters

$\begin{array}{llllllc}\text { atom } & \mathrm{U} 11 & \mathrm{U} 22 & \mathrm{U} 33 & \mathrm{U} 12 & \mathrm{U} 13 & \mathrm{U} 23 \\ \mathrm{Pt}(1) & 0.0128(2) & 0.0140(2) & 0.0086(2) & -0.00564(12) & -0.00335(11) & -0.00116(9) \\ \mathrm{Pd}(1) & 0.0128(2) & 0.0140(2) & 0.0086(2) & -0.00564(12) & -0.00335(11) & -0.00116(9) \\ \mathrm{P}(1) & 0.0177(6) & 0.0181(5) & 0.0120(4) & -0.0059(4) & -0.0056(4) & 0.0014(3) \\ \mathrm{Si}(1) & 0.0141(5) & 0.0173(5) & 0.0084(4) & -0.0069(4) & -0.0041(4) & -0.0017(3) \\ \mathrm{C}(1) & 0.021(2) & 0.025(2) & 0.010(2) & -0.009(2) & -0.006(1) & 0.0007(13) \\ \mathrm{C}(2) & 0.027(2) & 0.029(2) & 0.017(2) & -0.016(2) & -0.009(2) & 0.001(1) \\ \mathrm{C}(3) & 0.020(2) & 0.049(3) & 0.015(2) & -0.019(2) & -0.007(2) & 0.000(2) \\ \mathrm{C}(4) & 0.025(3) & 0.049(3) & 0.021(2) & -0.019(2) & -0.012(2) & 0.007(2) \\ \mathrm{C}(5) & 0.015(2) & 0.039(2) & 0.018(2) & -0.004(2) & -0.004(2) & 0.002(2) \\ \mathrm{C}(6) & 0.022(2) & 0.026(2) & 0.018(2) & -0.005(2) & -0.007(2) & -0.002(1) \\ \mathrm{C}(7) & 0.014(2) & 0.018(2) & 0.014(2) & -0.007(1) & -0.005(1) & -0.0002(12) \\ \mathrm{C}(8) & 0.014(2) & 0.027(2) & 0.014(2) & -0.004(2) & -0.001(1) & -0.009(1) \\ \mathrm{C}(9) & 0.028(2) & 0.033(2) & 0.012(2) & -0.013(2) & -0.002(2) & -0.008(1) \\ \mathrm{C}(10) & 0.014(2) & 0.030(2) & 0.024(2) & -0.010(2) & 0.007(2) & -0.011(2) \\ \mathrm{C}(11) & 0.016(2) & 0.026(2) & 0.031(2) & -0.002(2) & -0.006(2) & -0.003(2) \\ \mathrm{C}(12) & 0.011(2) & 0.025(2) & 0.015(2) & -0.004(2) & -0.006(1) & -0.0008(13) \\ \mathrm{C}(13) & 0.022(2) & 0.029(2) & 0.026(2) & -0.005(2) & -0.009(2) & 0.007(2) \\ \mathrm{C}(14) & 0.020(2) & 0.032(2) & 0.035(2) & 0.007(2) & -0.004(2) & -0.000(2)\end{array}$




$\begin{array}{lllllll}\mathrm{C}(15) & 0.027(2) & 0.032(2) & 0.016(2) & -0.006(2) & -0.010(2) & -0.001(2) \\ \mathrm{C}(16) & 0.029(3) & 0.030(2) & 0.022(2) & -0.011(2) & -0.003(2) & -0.006(2) \\ \mathrm{C}(17) & 0.023(2) & 0.025(2) & 0.027(2) & -0.009(2) & -0.013(2) & 0.001(2) \\ \mathrm{C}(18) & 0.084(5) & 0.031(3) & 0.070(4) & -0.026(3) & -0.031(4) & 0.005(3)\end{array}$

The general temperature factor expression: $\exp \left(-2 \pi^{2}\left(\mathrm{a}^{* 2} \mathrm{U}_{11} \mathrm{~h}^{2}+\mathrm{b}^{* 2} \mathrm{U}_{22} \mathrm{k}^{2}+\mathrm{c}^{* 2} \mathrm{U}_{33} \mathrm{l}^{2}+\right.\right.$ $\left.\left.2 a * b * U_{12} h k+2 a * c * U_{13} h l+2 b * c * U_{23} k l\right)\right)$

Table 3. Bond lengths $(\AA)$

$\begin{array}{llllll}\text { atom } & \text { atom } & \text { distance } & \text { atom } & \text { atom } & \text { distance } \\ \mathrm{Pt}(1) & \mathrm{P}(1) & 2.2890(10) & \mathrm{Pt}(1) & \mathrm{Si}(1) & 2.3160(10) \\ \mathrm{Pd}(1) & \mathrm{P}(1) & 2.2890(10) & \mathrm{Pd}(1) & \mathrm{Si}(1) & 2.3160(10) \\ \mathrm{P}(1) & \mathrm{C}(13) & 1.825(4) & \mathrm{P}(1) & \mathrm{C}(15) & 1.838(4) \\ \mathrm{P}(1) & \mathrm{C}(17) & 1.827(4) & \mathrm{Si}(1) & \mathrm{C}(1) & 1.871(4) \\ \mathrm{Si}(1) & \mathrm{C}(7) & 1.889(4) & \mathrm{C}(1) & \mathrm{C}(2) & 1.404(5) \\ \mathrm{C}(1) & \mathrm{C}(6) & 1.399(5) & \mathrm{C}(2) & \mathrm{C}(3) & 1.405(6) \\ \mathrm{C}(3) & \mathrm{C}(4) & 1.387(6) & \mathrm{C}(4) & \mathrm{C}(5) & 1.382(7) \\ \mathrm{C}(5) & \mathrm{C}(6) & 1.386(6) & \mathrm{C}(7) & \mathrm{C}(8) & 1.400(5) \\ \mathrm{C}(7) & \mathrm{C}(12) & 1.409(5) & \mathrm{C}(8) & \mathrm{C}(9) & 1.394(5) \\ \mathrm{C}(9) & \mathrm{C}(10) & 1.376(6) & \mathrm{C}(10) & \mathrm{C}(11) & 1.409(6) \\ \mathrm{C}(11) & \mathrm{C}(12) & 1.392(5) & \mathrm{C}(13) & \mathrm{C}(14) & 1.519(6) \\ \mathrm{C}(15) & \mathrm{C}(16) & 1.524(6) & \mathrm{C}(17) & \mathrm{C}(18) & 1.478(7)\end{array}$

Table 4. Bond lengths involving hydrogens $(\AA)$

$\begin{array}{llllll}\text { atom } & \text { atom } & \text { distance } & \text { atom } & \text { atom } & \begin{array}{l}\text { distance } \\ \mathrm{C}(2)\end{array} \\ \mathrm{H}(1) & 0.95(3) & \mathrm{C}(3) & \mathrm{H}(2) & 0.950(12) \\ \mathrm{C}(4) & \mathrm{H}(3) & 0.950(12) & \mathrm{C}(5) & \mathrm{H}(4) & 0.95(3) \\ \mathrm{C}(6) & \mathrm{H}(5) & 0.950(12) & \mathrm{C}(8) & \mathrm{H}(6) & 0.95(2) \\ \mathrm{C}(9) & \mathrm{H}(7) & 0.950(10) & \mathrm{C}(10) & \mathrm{H}(8) & 0.95(3) \\ \mathrm{C}(11) & \mathrm{H}(9) & 0.95(2) & \mathrm{C}(12) & \mathrm{H}(10) & 0.950(9) \\ \mathrm{C}(13) & \mathrm{H}(11) & 0.95(2) & \mathrm{C}(13) & \mathrm{H}(12) & 0.950(11) \\ \mathrm{C}(14) & \mathrm{H}(13) & 0.95(1) & \mathrm{C}(14) & \mathrm{H}(14) & 0.95(2) \\ \mathrm{C}(14) & \mathrm{H}(15) & 0.950(10) & \mathrm{C}(15) & \mathrm{H}(16) & 0.95(2) \\ \mathrm{C}(15) & \mathrm{H}(17) & 0.950(11) & \mathrm{C}(16) & \mathrm{H}(18) & 0.950(13) \\ \mathrm{C}(16) & \mathrm{H}(19) & 0.950(12) & \mathrm{C}(16) & \mathrm{H}(20) & 0.95(2) \\ \mathrm{C}(17) & \mathrm{H}(21) & 0.95(2) & \mathrm{C}(17) & \mathrm{H}(22) & 0.95(2) \\ \mathrm{C}(18) & \mathrm{H}(23) & 0.95(3) & \mathrm{C}(18) & \mathrm{H}(24) & 0.95(2) \\ \mathrm{C}(18) & \mathrm{H}(25) & 0.95(2) & & & \end{array}$


Table 5. Bond angles $\left({ }^{\circ}\right)$

$\begin{array}{llllllll}\text { atom } & \text { atom } & \text { atom } & \text { angle } & \text { atom } & \text { atom } & \text { atom } & \text { angle } \\ \mathrm{P}(1) & \mathrm{Pt}(1) & \mathrm{Si}(1) & 102.16(4) & \mathrm{P}(1) & \mathrm{Pd}(1) & \mathrm{Si}(1) & 102.16(4) \\ \mathrm{Pt}(1) & \mathrm{P}(1) & \mathrm{C}(13) & 114.6(1) & \mathrm{Pd}(1) & \mathrm{P}(1) & \mathrm{C}(13) & 114.6(1) \\ \mathrm{Pt}(1) & \mathrm{P}(1) & \mathrm{C}(15) & 118.9(1) & \mathrm{Pd}(1) & \mathrm{P}(1) & \mathrm{C}(15) & 118.9(1) \\ \mathrm{C}(13) & \mathrm{P}(1) & \mathrm{C}(15) & 101.7(2) & \mathrm{Pt}(1) & \mathrm{P}(1) & \mathrm{C}(17) & 113.10(13) \\ \mathrm{Pd}(1) & \mathrm{P}(1) & \mathrm{C}(17) & 113.10(13) & \mathrm{C}(13) & \mathrm{P}(1) & \mathrm{C}(17) & 104.6(2) \\ \mathrm{C}(15) & \mathrm{P}(1) & \mathrm{C}(17) & 102.2(2) & \mathrm{Pt}(1) & \mathrm{Si}(1) & \mathrm{C}(1) & 117.44(13) \\ \mathrm{Pd}(1) & \mathrm{Si}(1) & \mathrm{C}(1) & 117.44(13) & \mathrm{Pt}(1) & \mathrm{Si}(1) & \mathrm{C}(7) & 116.84(12) \\ \mathrm{Pd}(1) & \mathrm{Si}(1) & \mathrm{C}(7) & 116.84(12) & \mathrm{C}(1) & \mathrm{Si}(1) & \mathrm{C}(7) & 108.1(2) \\ \mathrm{Si}(1) & \mathrm{C}(1) & \mathrm{C}(2) & 122.1(3) & \mathrm{Si}(1) & \mathrm{C}(1) & \mathrm{C}(6) & 120.5(3) \\ \mathrm{C}(2) & \mathrm{C}(1) & \mathrm{C}(6) & 117.1(4) & \mathrm{C}(1) & \mathrm{C}(2) & \mathrm{C}(3) & 121.2(4) \\ \mathrm{C}(2) & \mathrm{C}(3) & \mathrm{C}(4) & 119.5(4) & \mathrm{C}(3) & \mathrm{C}(4) & \mathrm{C}(5) & 120.4(4) \\ \mathrm{C}(4) & \mathrm{C}(5) & \mathrm{C}(6) & 119.7(5) & \mathrm{C}(1) & \mathrm{C}(6) & \mathrm{C}(5) & 122.2(4) \\ \mathrm{Si}(1) & \mathrm{C}(7) & \mathrm{C}(8) & 123.4(3) & \mathrm{Si}(1) & \mathrm{C}(7) & \mathrm{C}(12) & 119.6(3) \\ \mathrm{C}(8) & \mathrm{C}(7) & \mathrm{C}(12) & 116.8(3) & \mathrm{C}(7) & \mathrm{C}(8) & \mathrm{C}(9) & 121.6(4) \\ \mathrm{C}(8) & \mathrm{C}(9) & \mathrm{C}(10) & 120.3(4) & \mathrm{C}(9) & \mathrm{C}(10) & \mathrm{C}(11) & 120.2(4) \\ \mathrm{C}(10) & \mathrm{C}(11) & \mathrm{C}(12) & 118.6(4) & \mathrm{C}(7) & \mathrm{C}(12) & \mathrm{C}(11) & 122.4(4) \\ \mathrm{P}(1) & \mathrm{C}(13) & \mathrm{C}(14) & 113.8(3) & \mathrm{P}(1) & \mathrm{C}(15) & \mathrm{C}(16) & 112.5(3) \\ \mathrm{P}(1) & \mathrm{C}(17) & \mathrm{C}(18) & 114.3(4) & & & & \end{array}$

Table 6. Bond angles involving hydrogens $\left({ }^{\circ}\right)$

$\begin{array}{llllllll}\text { atom } & \text { atom } & \text { atom } & \text { angle } & \text { atom } & \text { atom } & \text { atom } & \text { angle } \\ \mathrm{C}(1) & \mathrm{C}(2) & \mathrm{H}(1) & 119.4(5) & \mathrm{C}(3) & \mathrm{C}(2) & \mathrm{H}(1) & 119.4(5) \\ \mathrm{C}(2) & \mathrm{C}(3) & \mathrm{H}(2) & 120.2(5) & \mathrm{C}(4) & \mathrm{C}(3) & \mathrm{H}(2) & 120.3(6) \\ \mathrm{C}(3) & \mathrm{C}(4) & \mathrm{H}(3) & 119.2(6) & \mathrm{C}(5) & \mathrm{C}(4) & \mathrm{H}(3) & 120.5(6) \\ \mathrm{C}(4) & \mathrm{C}(5) & \mathrm{H}(4) & 119.6(6) & \mathrm{C}(6) & \mathrm{C}(5) & \mathrm{H}(4) & 120.7(6) \\ \mathrm{C}(1) & \mathrm{C}(6) & \mathrm{H}(5) & 118.7(5) & \mathrm{C}(5) & \mathrm{C}(6) & \mathrm{H}(5) & 119.1(5) \\ \mathrm{C}(7) & \mathrm{C}(8) & \mathrm{H}(6) & 118.7(5) & \mathrm{C}(9) & \mathrm{C}(8) & \mathrm{H}(6) & 119.7(5) \\ \mathrm{C}(8) & \mathrm{C}(9) & \mathrm{H}(7) & 119.7(5) & \mathrm{C}(10) & \mathrm{C}(9) & \mathrm{H}(7) & 120.0(5) \\ \mathrm{C}(9) & \mathrm{C}(10) & \mathrm{H}(8) & 120.1(5) & \mathrm{C}(11) & \mathrm{C}(10) & \mathrm{H}(8) & 119.7(5) \\ \mathrm{C}(10) & \mathrm{C}(11) & \mathrm{H}(9) & 120.6(6) & \mathrm{C}(12) & \mathrm{C}(11) & \mathrm{H}(9) & 120.8(6) \\ \mathrm{C}(7) & \mathrm{C}(12) & \mathrm{H}(10) & 119.1(5) & \mathrm{C}(11) & \mathrm{C}(12) & \mathrm{H}(10) & 118.5(5) \\ \mathrm{P}(1) & \mathrm{C}(13) & \mathrm{H}(11) & 108.2(5) & \mathrm{C}(14) & \mathrm{C}(13) & \mathrm{H}(11) & 108.7(5) \\ \mathrm{P}(1) & \mathrm{C}(13) & \mathrm{H}(12) & 108.5(5) & \mathrm{C}(14) & \mathrm{C}(13) & \mathrm{H}(12) & 108.1(5) \\ \mathrm{H}(11) & \mathrm{C}(13) & \mathrm{H}(12) & 109.5(6) & \mathrm{C}(13) & \mathrm{C}(14) & \mathrm{H}(13) & 109.7(6) \\ \mathrm{C}(13) & \mathrm{C}(14) & \mathrm{H}(14) & 109.5(6) & \mathrm{H}(13) & \mathrm{C}(14) & \mathrm{H}(14) & 109.5(7) \\ \mathrm{C}(13) & \mathrm{C}(14) & \mathrm{H}(15) & 109.1(6) & \mathrm{H}(13) & \mathrm{C}(14) & \mathrm{H}(15) & 109.5(7) \\ \mathrm{H}(14) & \mathrm{C}(14) & \mathrm{H}(15) & 109.5(7) & \mathrm{P}(1) & \mathrm{C}(15) & \mathrm{H}(16) & 108.7(5) \\ \mathrm{C}(16) & \mathrm{C}(15) & \mathrm{H}(16) & 109.1(5) & \mathrm{P}(1) & \mathrm{C}(15) & \mathrm{H}(17) & 108.6(5) \\ \mathrm{C}(16) & \mathrm{C}(15) & \mathrm{H}(17) & 108.4(5) & \mathrm{H}(16) & \mathrm{C}(15) & \mathrm{H}(17) & 109.5(6) \\ \mathrm{C}(15) & \mathrm{C}(16) & \mathrm{H}(18) & 109.8(5) & \mathrm{C}(15) & \mathrm{C}(16) & \mathrm{H}(19) & 109.6(5)\end{array}$




$\begin{array}{llllllll}\mathrm{H}(18) & \mathrm{C}(16) & \mathrm{H}(19) & 109.5(6) & \mathrm{C}(15) & \mathrm{C}(16) & \mathrm{H}(20) & 109.0(5) \\ \mathrm{H}(18) & \mathrm{C}(16) & \mathrm{H}(20) & 109.5(6) & \mathrm{H}(19) & \mathrm{C}(16) & \mathrm{H}(20) & 109.5(6) \\ \mathrm{P}(1) & \mathrm{C}(17) & \mathrm{H}(21) & 108.5(5) & \mathrm{C}(18) & \mathrm{C}(17) & \mathrm{H}(21) & 108.6(6) \\ \mathrm{P}(1) & \mathrm{C}(17) & \mathrm{H}(22) & 108.1(5) & \mathrm{C}(18) & \mathrm{C}(17) & \mathrm{H}(22) & 107.8(6) \\ \mathrm{H}(21) & \mathrm{C}(17) & \mathrm{H}(22) & 109.5(6) & \mathrm{C}(17) & \mathrm{C}(18) & \mathrm{H}(23) & 110.0(8) \\ \mathrm{C}(17) & \mathrm{C}(18) & \mathrm{H}(24) & 109.4(8) & \mathrm{H}(23) & \mathrm{C}(18) & \mathrm{H}(24) & 109.5(10) \\ \mathrm{C}(17) & \mathrm{C}(18) & \mathrm{H}(25) & 109.0(8) & \mathrm{H}(23) & \mathrm{C}(18) & \mathrm{H}(25) & 109.5(10) \\ \mathrm{H}(24) & \mathrm{C}(18) & \mathrm{H}(25) & 109.5(10) & & & & \end{array}$




\section{Crystallographic Results of complex 2}

\section{Data Collection}

\section{Experimental}

A colorless prism crystal of $\mathrm{C}_{42} \mathrm{H}_{65} \mathrm{P}_{3} \mathrm{PdPtSi}_{2}$ having approximate dimensions of $0.20 \mathrm{x}$ $0.20 \times 0.20 \mathrm{~mm}$ was mounted on a glass fiber. All measurements were made on a unknown unknown diffractometer with graphite monochromated Mo-K• radiation.

Cell constants and an orientation matrix for data collection, obtained from a least-squares refinement using the setting angles of 13707 carefully centered reflections in the range $6.04<2 \bullet$ $<54.97^{\circ}$ corresponded to a primitive triclinic cell with dimensions:

$$
\begin{array}{ll}
\mathrm{a}=11.8945(5) \AA & \alpha=65.69(1)^{\mathrm{O}} \\
\mathrm{b}=20.3726(8) \AA & \beta=73.11(2)^{\mathrm{O}} \\
\mathrm{c}=21.3757(7) \AA & \gamma=78.03(2)^{\mathrm{O}} \\
\mathrm{V}=4494.1(3) \AA^{3} &
\end{array}
$$

For $\mathrm{Z}=4$ and F.W. $=1020.56$, the calculated density is $1.51 \mathrm{~g} / \mathrm{cm}^{3}$. Based on a statistical analysis of intensity distribution, and the successful solution and refinement of the structure, the space group was determined to be:

$$
\text { P-1 (\#2) }
$$

The data were collected at a temperature of $20 \pm 1^{\circ} \mathrm{C}$ using the $\omega$ - $2 \theta$ scan technique to a maximum $2 \theta$ value of $55.0^{\circ}$. Omega scans of several intense reflections, made prior to data collection, had an average width at half-height of $0.00^{\circ}$ with a take-off angle of $6.0^{\circ}$. Scans of $(0.00+0.00 \tan \theta)^{\mathrm{O}}$ were made at a speed of $0.0^{\circ} / \mathrm{min}$ (in $\omega$ ).

\section{Data Reduction}

Of the 32136 reflections that were collected, 18826 were unique $\left(\mathrm{R}_{\mathrm{int}}=0.042\right)$; equivalent reflections were merged. No decay correction was applied.

The linear absorption coefficient, $\mu$, for Mo-K $\alpha$ radiation is $36.8 \mathrm{~cm}^{-1}$. The data were corrected for Lorentz and polarization effects.

\section{$\underline{\text { Structure Solution and Refinement }}$}

The structure was solved by heavy-atom Patterson methods ${ }^{1}$ and expanded using Fourier techniques ${ }^{2}$. The non-hydrogen atoms were refined anisotropically. Hydrogen atoms were refined using the riding model. The final cycle of full-matrix least-squares refinement ${ }^{3}$ on $\mathrm{F}$ was based on 13764 observed reflections (I > 3.00б(I)) and 1021 variable parameters and converged (largest parameter shift was 3.39 times its esd) with unweighted and weighted agreement factors of:

$$
\begin{gathered}
\mathrm{R}=\Sigma\|\mathrm{Fo}|-| \mathrm{Fc}\| / \Sigma|\mathrm{Fo}|=0.066 \\
\mathrm{R}_{\mathrm{W}}=\left[\Sigma \mathrm{w}(|\mathrm{Fo}|-|\mathrm{Fc}|)^{2} / \Sigma \mathrm{w} \mathrm{Fo}^{2}\right]^{1 / 2}=0.081
\end{gathered}
$$


The standard deviation of an observation of unit weight ${ }^{4}$ was 2.17 . Unit weights were used. Plots of $\Sigma \mathrm{w}(|\mathrm{Fo}|-|\mathrm{Fc}|)^{2}$ versus $|\mathrm{Fo}|$, reflection order in data collection, $\sin \theta / \lambda$ and various classes of indices showed no unusual trends. The maximum and minimum peaks on the final difference Fourier map corresponded to 4.99 and -4.44 e/ $\AA^{3}$, respectively.

Neutral atom scattering factors were taken from Cromer and Waber ${ }^{5}$. Anomalous dispersion effects were included in Fcalc 6 ; the values for $\Delta \mathrm{f}^{\prime}$ and $\Delta \mathrm{f}^{\prime \prime}$ were those of Creagh and McAuley 7 . The values for the mass attenuation coefficients are those of Creagh and Hubbell 8 . All calculations were performed using the CrystalStructure 9,10 crystallographic software package.

\section{References}

(1) PATTY: Beurskens, P.T., Admiraal, G., Beurskens, G., Bosman, W.P., Garcia-Granda, S., Gould, R.O., Smits, J.M.M. and Smykalla, C. (1992). The DIRDIF program system, Technical Report of the Crystallography Laboratory, University of Nijmegen, The Netherlands.

(2) DIRDIF99: Beurskens, P.T., Admiraal, G., Beurskens, G., Bosman, W.P., de Gelder, R., Israel, R. and Smits, J.M.M.(1999). The DIRDIF-99 program system, Technical Report of the Crystallography Laboratory, University of Nijmegen, The Netherlands.

(3) Least Squares function minimized:

$$
\Sigma w\left(\left|\mathrm{~F}_{\mathrm{O}}\right|-\left|\mathrm{F}_{\mathrm{C}}\right|\right)^{2} \quad \text { where } \mathrm{w}=\text { Least Squares weights. }
$$

(4) Standard deviation of an observation of unit weight:

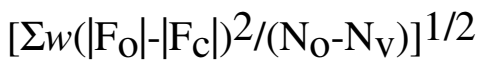

$$
\begin{aligned}
& \text { where: } \quad \mathrm{N}_{\mathrm{O}} \text { = number of observations } \\
& \mathrm{N}_{\mathrm{V}}=\text { number of variables }
\end{aligned}
$$

(5) Cromer, D. T. \& Waber, J. T.; "International Tables for X-ray Crystallography", Vol. IV, The Kynoch Press, Birmingham, England, Table 2.2 A (1974).

(6) Ibers, J. A. \& Hamilton, W. C.; Acta Crystallogr., 17, 781 (1964).

(7) Creagh, D. C. \& McAuley, W.J .; "International Tables for Crystallography", Vol C, (A.J.C. Wilson, ed.), Kluwer Academic Publishers, Boston, Table 4.2.6.8, pages 219-222 (1992).

(8) Creagh, D. C. \& Hubbell, J.H..; "International Tables for Crystallography", Vol C, (A.J.C. Wilson, ed.), Kluwer Academic Publishers, Boston, Table 4.2.4.3, pages 200-206 (1992).

(9) CrystalStructure 3.10: Crystal Structure Analysis Package, Rigaku and Rigaku/MSC (2000-2002). 
(10) CRYSTALS Issue 10: Watkin, D.J., Prout, C.K. Carruthers, J.R. \& Betteridge, P.W. Chemical Crystallography Laboratory, Oxford, UK.

\section{EXPERIMENTAL DETAILS}

\section{A. Crystal Data}

Empirical Formula

Formula Weight

Crystal Color, Habit

Crystal Dimensions

Crystal System

Lattice Type

No. of Reflections Used for Unit

Cell Determination $(2 \bullet$ range)

Omega Scan Peak Width

at Half-height

Lattice Parameters
Space Group

$Z$ value

Dcalc

F000

$\mu(\mathrm{MoK} \bullet)$
$\mathrm{C}_{42} \mathrm{H}_{65} \mathrm{P}_{3} \mathrm{PdPtSi}_{2}$

1020.56

colorless, prism

$0.20 \times 0.20 \times 0.20 \mathrm{~mm}$

triclinic

Primitive

$13707\left(6.0-55.0^{\circ}\right)$

$0.00^{\circ}$

$\mathrm{a}=11.8945(5) \AA$

$\mathrm{b}=20.3726(8) \AA$

$\mathrm{c}=21.3757(7) \AA$

$\alpha=65.69(1)^{\mathrm{O}}$

$\beta=73.11(2)^{\circ}$

$\gamma=78.03(2)^{\mathrm{o}}$

$\mathrm{V}=4494.1(3) \AA^{3}$

P-1 (\#2)

4

$1.508 \mathrm{~g} / \mathrm{cm}^{3}$

2056.00

$36.84 \mathrm{~cm}^{-1}$

B. Intensity Measurements

Diffractometer

Radiation

Take-off Angle

Detector Aperture

Crystal to Detector Distance

Voltage, Current

Temperature

Scan Type

Scan Rate

Scan Width unknown

$\operatorname{MoK} \alpha(\lambda=0.71070 \AA)$

graphite monochromated

$2.8^{0}$

2.0 - $2.5 \mathrm{~mm}$ horizontal

$2.0 \mathrm{~mm}$ vertical

$21 \mathrm{~mm}$

$50 \mathrm{kV}, 40 \mathrm{~mA}$

$20.0^{\circ} \mathrm{C}$

$\omega-2 \theta$

$0.0^{\mathrm{O}} / \mathrm{min}$ (in $\omega$ ) (up to 0 scans)

$(0.00+0.00 \tan \theta)^{\mathrm{O}}$ 
$2 \theta_{\max }$

No. of Reflections Measured

Corrections $55.0^{\circ}$

Total: 32136

Unique: $18826\left(\mathrm{R}_{\text {int }}=0.042\right)$

Lorentz-polarization

C. Structure Solution and Refinement

Structure Solution

Refinement

Function Minimized

Least Squares Weights

Anomalous Dispersion

No. Observations $(\mathrm{I}>3.00 \sigma(\mathrm{I}))$

No. Variables

Reflection/Parameter Ratio

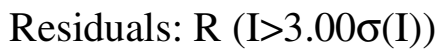

Residuals: Rw (I>3.00б(I))

Goodness of Fit Indicator

Max Shift/Error in Final Cycle

Maximum peak in Final Diff. Map

Minimum peak in Final Diff. Map
Patterson Methods (DIRDIF99 PATTY)

Full-matrix least-squares on $\mathrm{F}$

$\Sigma_{\mathrm{w}}(|\mathrm{Fo}|-|\mathrm{Fc}|)^{2}$

1

All non-hydrogen atoms

13764

1021

13.48

0.066

0.081

2.17

3.39

$4.99 \mathrm{e} / \AA^{3}$

$-4.44 \mathrm{e} / \AA^{3}$

Table 1. Atomic coordinates and $\mathrm{B}_{\mathrm{iso}} / \mathrm{B}_{\mathrm{eq}}$

$\begin{array}{lclll}\text { atom } & \mathrm{x} & \mathrm{y} & \mathrm{z} & \mathrm{B}_{\mathrm{eq}} \\ \mathrm{Pt}(1) & 0.03517(4) & 0.80647(2) & 0.79393(3) & 1.599(9) \\ \mathrm{Pt}(2) & 0.67847(5) & 0.69210(3) & 0.35777(3) & 2.061(11) \\ \mathrm{Pd}(1) & -0.05704(7) & 0.67559(4) & 0.87331(4) & 0.97(2) \\ \mathrm{Pd}(2) & 0.59088(9) & 0.82316(5) & 0.27202(5) & 1.68(2) \\ \mathrm{P}(1) & -0.2040(3) & 0.6007(2) & 0.9292(2) & 1.72(6) \\ \mathrm{P}(2) & 0.1217(3) & 0.8153(2) & 0.6756(2) & 1.98(6) \\ \mathrm{P}(3) & 0.0923(3) & 0.8907(2) & 0.8232(2) & 1.83(6) \\ \mathrm{P}(4) & 0.4747(3) & 0.9154(2) & 0.2094(2) & 2.39(7) \\ \mathrm{P}(5) & 0.7048(4) & 0.6528(2) & 0.4704(2) & 3.19(9) \\ \mathrm{P}(6) & 0.8037(4) & 0.6284(2) & 0.2883(2) & 2.42(7) \\ \mathrm{Si}(1) & -0.1669(3) & 0.7813(2) & 0.8212(2) & 1.42(6) \\ \mathrm{Si}(2) & 0.1387(3) & 0.6998(2) & 0.8591(2) & 1.68(7) \\ \mathrm{Si}(3) & 0.7696(3) & 0.7992(2) & 0.3127(2) & 2.11(7) \\ \mathrm{Si}(4) & 0.4891(4) & 0.7209(2) & 0.3296(2) & 2.23(8) \\ \mathrm{C}(1) & -0.2325(12) & 0.7889(7) & 0.7489(6) & 2.1(3)\end{array}$




\begin{tabular}{|c|c|c|c|c|}
\hline$C(2)$ & $-0.2050(13)$ & $0.7356(8)$ & $0.7187(8)$ & $2.7(3)$ \\
\hline$C(3)$ & $-0.243(2)$ & $0.7430(9)$ & $0.6618(8)$ & $3.4(4)$ \\
\hline$C(4)$ & $-0.309(2)$ & $0.8031(10)$ & $0.6285(8)$ & $4.1(4)$ \\
\hline$C(5)$ & $-0.343(2)$ & $0.8591(9)$ & $0.6574(8)$ & $3.5(4)$ \\
\hline$C(6)$ & $-0.3004(12)$ & $0.8496(7)$ & $0.7147(7)$ & $2.2(3)$ \\
\hline $\mathrm{C}(7)$ & $-0.2944(10)$ & $0.8064(6)$ & $0.8873(6)$ & $1.6(2)$ \\
\hline $\mathrm{C}(8)$ & $-0.4136(12)$ & $0.8063(7)$ & $0.8878(7)$ & $2.2(3)$ \\
\hline $\mathrm{C}(9)$ & $-0.5046(12)$ & $0.8163(7)$ & $0.9421(8)$ & $2.6(3)$ \\
\hline$C(10)$ & $-0.4844(12)$ & $0.8276(8)$ & $0.9965(8)$ & $2.6(3)$ \\
\hline $\mathrm{C}(11)$ & $-0.367(1)$ & $0.8271(8)$ & $0.9972(8)$ & $2.9(3)$ \\
\hline$C(12)$ & $-0.2753(11)$ & $0.8165(7)$ & $0.9428(7)$ & $2.0(3)$ \\
\hline $\mathrm{C}(13)$ & $0.2780(11)$ & $0.6566(7)$ & $0.8137(7)$ & $2.1(3)$ \\
\hline $\mathrm{C}(14)$ & $0.2850(13)$ & $0.5865(7)$ & $0.8191(8)$ & $2.6(3)$ \\
\hline$C(15)$ & $0.389(1)$ & $0.5522(9)$ & $0.7884(9)$ & $3.5(4)$ \\
\hline$C(16)$ & $0.487(2)$ & $0.5892(10)$ & $0.7518(10)$ & $4.3(4)$ \\
\hline$C(17)$ & $0.480(2)$ & $0.6610(9)$ & $0.7480(11)$ & $4.2(4)$ \\
\hline $\mathrm{C}(18)$ & $0.3768(13)$ & $0.6939(8)$ & $0.7782(9)$ & $3.1(3)$ \\
\hline$C(19)$ & $0.1818(10)$ & $0.6965(6)$ & $0.9391(7)$ & $1.7(2)$ \\
\hline$C(20)$ & $0.0946(12)$ & $0.7187(7)$ & $0.9899(7)$ & $2.1(3)$ \\
\hline $\mathrm{C}(21)$ & $0.122(1)$ & $0.7197(8)$ & $1.0481(9)$ & $3.2(3)$ \\
\hline $\mathrm{C}(22)$ & $0.2379(11)$ & $0.6957(6)$ & $1.0580(7)$ & $1.9(3)$ \\
\hline $\mathrm{C}(23)$ & $0.3223(13)$ & $0.6731(8)$ & $1.0097(9)$ & $3.0(3)$ \\
\hline $\mathrm{C}(24)$ & $0.2940(12)$ & $0.6736(7)$ & $0.9520(8)$ & $2.2(3)$ \\
\hline$C(25)$ & $-0.1510(13)$ & $0.5053(7)$ & $0.9609(8)$ & $2.8(3)$ \\
\hline$C(26)$ & $-0.083(2)$ & $0.4804(8)$ & $0.8998(11)$ & $4.4(4)$ \\
\hline$C(27)$ & $-0.3131(12)$ & $0.6047(7)$ & $0.8832(7)$ & $2.0(3)$ \\
\hline$C(28)$ & $-0.4068(13)$ & $0.5517(8)$ & $0.9207(9)$ & $2.9(3)$ \\
\hline $\mathrm{C}(29)$ & $-0.296(1)$ & $0.6132(8)$ & $1.0104(8)$ & $2.9(3)$ \\
\hline$C(30)$ & $-0.221(2)$ & $0.6140(9)$ & $1.0575(9)$ & $4.1(4)$ \\
\hline $\mathrm{C}(31)$ & $0.2487(13)$ & $0.8685(8)$ & $0.6274(8)$ & $3.0(3)$ \\
\hline$C(32)$ & $0.287(2)$ & $0.8877(8)$ & $0.5475(9)$ & $3.9(4)$ \\
\hline $\mathrm{C}(33)$ & $0.1856(13)$ & $0.7322(8)$ & $0.6589(8)$ & $2.8(3)$ \\
\hline $\mathrm{C}(34)$ & $0.1037(13)$ & $0.6720(7)$ & $0.6907(8)$ & $2.7(3)$ \\
\hline$C(35)$ & $0.0229(13)$ & $0.8572(7)$ & $0.6151(7)$ & $2.3(3)$ \\
\hline$C(36)$ & $-0.029(2)$ & $0.9356(8)$ & $0.6056(9)$ & $3.5(4)$ \\
\hline$C(37)$ & $0.0854(11)$ & $0.9847(6)$ & $0.7583(7)$ & $2.1(3)$ \\
\hline $\mathrm{C}(38)$ & $-0.0368(13)$ & $1.0189(7)$ & $0.7511(9)$ & $3.0(3)$ \\
\hline C(39) & $0.2402(12)$ & $0.8818(7)$ & $0.8373(8)$ & $2.5(3)$ \\
\hline$C(40)$ & $0.3388(13)$ & $0.8944(8)$ & $0.7709(9)$ & $3.2(4)$ \\
\hline $\mathrm{C}(41)$ & $-0.0032(13)$ & $0.9031(7)$ & $0.9038(8)$ & $2.6(3)$ \\
\hline$C(42)$ & $0.022(2)$ & $0.9546(10)$ & $0.9311(10)$ & $4.8(5)$ \\
\hline$C(43)$ & $0.928(1)$ & $0.8023(7)$ & $0.2569(8)$ & $2.8(3)$ \\
\hline $\mathrm{C}(44)$ & $0.953(1)$ & $0.8460(8)$ & $0.1873(8)$ & $2.9(3)$ \\
\hline$C(45)$ & $1.066(2)$ & $0.8449(9)$ & $0.1454(10)$ & $3.8(4)$ \\
\hline$C(46)$ & $1.156(2)$ & $0.8005(12)$ & $0.1750(13)$ & $4.9(5)$ \\
\hline
\end{tabular}




\begin{tabular}{|c|c|c|c|c|}
\hline $\mathrm{C}(47)$ & $1.135(2)$ & $0.7583(11)$ & $0.2443(12)$ & $4.5(5)$ \\
\hline $\mathrm{C}(48)$ & $1.020(1)$ & $0.7584(9)$ & $0.2850(10)$ & $3.5(4)$ \\
\hline C(49) & $0.7632(13)$ & $0.8486(6)$ & $0.3733(7)$ & $2.2(3)$ \\
\hline$C(50)$ & $0.662(2)$ & $0.8911(9)$ & $0.3894(9)$ & $3.4(4)$ \\
\hline $\mathrm{C}(51)$ & $0.651(2)$ & $0.9294(8)$ & $0.4330(9)$ & $3.6(4)$ \\
\hline $\mathrm{C}(52)$ & $0.743(2)$ & $0.9264(8)$ & $0.4592(9)$ & $3.7(4)$ \\
\hline $\mathrm{C}(53)$ & $0.848(2)$ & $0.8860(8)$ & $0.4437(8)$ & $3.2(4)$ \\
\hline $\mathrm{C}(54)$ & $0.856(1)$ & $0.8481(8)$ & $0.3992(9)$ & $3.0(3)$ \\
\hline$C(55)$ & $0.3449(13)$ & $0.7248(8)$ & $0.3972(7)$ & $2.7(3)$ \\
\hline$C(56)$ & $0.259(2)$ & $0.7832(11)$ & $0.3767(10)$ & $4.6(4)$ \\
\hline $\mathrm{C}(57)$ & $0.153(2)$ & $0.7885(13)$ & $0.4223(12)$ & $5.7(6)$ \\
\hline $\mathrm{C}(58)$ & $0.126(2)$ & $0.7355(12)$ & $0.4897(11)$ & $4.6(5)$ \\
\hline C(59) & $0.206(2)$ & $0.6801(11)$ & $0.5099(10)$ & $4.6(5)$ \\
\hline$C(60)$ & $0.317(2)$ & $0.6749(9)$ & $0.4636(9)$ & $3.9(4)$ \\
\hline $\mathrm{C}(61)$ & $0.350(2)$ & $0.9602(10)$ & $0.2601(10)$ & $4.0(4)$ \\
\hline$C(62)$ & $0.376(2)$ & $0.9564(9)$ & $0.3256(10)$ & $4.1(4)$ \\
\hline $\mathrm{C}(63)$ & $0.558(2)$ & $0.9922(8)$ & $0.1474(10)$ & $4.2(4)$ \\
\hline $\mathrm{C}(64)$ & $0.657(2)$ & $0.9728(11)$ & $0.0933(10)$ & $4.7(5)$ \\
\hline$C(65)$ & $0.402(1)$ & $0.8987(8)$ & $0.1530(8)$ & $2.7(3)$ \\
\hline $\mathrm{C}(66)$ & $0.339(2)$ & $0.9639(9)$ & $0.1057(8)$ & $3.9(4)$ \\
\hline $\mathrm{C}(67)$ & $0.645(2)$ & $0.7086(9)$ & $0.5229(9)$ & $3.6(4)$ \\
\hline $\mathrm{C}(68)$ & $0.518(2)$ & $0.733(1)$ & $0.5285(12)$ & $6.1(7)$ \\
\hline $\mathrm{C}(69)$ & $0.856(2)$ & $0.6331(11)$ & $0.4805(10)$ & $5.2(5)$ \\
\hline $\mathrm{C}(70)$ & $0.882(3)$ & $0.593(1)$ & $0.5495(13)$ & $8.2(8)$ \\
\hline $\mathrm{C}(71)$ & $0.640(3)$ & $0.5695(12)$ & $0.5323(11)$ & $9.6(9)$ \\
\hline $\mathrm{C}(72)$ & $0.675(5)$ & $0.508(1)$ & $0.511(2)$ & $18.3(17)$ \\
\hline $\mathrm{C}(73)$ & $0.931(2)$ & $0.5698(8)$ & $0.3222(9)$ & $3.5(4)$ \\
\hline $\mathrm{C}(74)$ & $1.015(2)$ & $0.5278(10)$ & $0.2803(10)$ & $4.6(5)$ \\
\hline$C(75)$ & $0.732(2)$ & $0.5663(7)$ & $0.2725(8)$ & $3.0(3)$ \\
\hline$C(76)$ & $0.675(2)$ & $0.5062(9)$ & $0.3379(9)$ & $4.2(4)$ \\
\hline $\mathrm{C}(77)$ & $0.8813(13)$ & $0.6783(8)$ & $0.1971(8)$ & $2.8(3)$ \\
\hline $\mathrm{C}(78)$ & $0.8014(13)$ & $0.7361(8)$ & $0.1526(8)$ & $2.7(3)$ \\
\hline $\mathrm{C}(79)$ & $0.4487(13)$ & $0.6783(7)$ & $0.2757(7)$ & $2.5(3)$ \\
\hline $\mathrm{C}(80)$ & $0.380(2)$ & $0.6216(8)$ & $0.3085(9)$ & $3.6(4)$ \\
\hline $\mathrm{C}(81)$ & $0.357(2)$ & $0.5860(9)$ & $0.2683(9)$ & $4.2(4)$ \\
\hline $\mathrm{C}(82)$ & $0.400(1)$ & $0.6092(7)$ & $0.1971(8)$ & $2.9(3)$ \\
\hline $\mathrm{C}(83)$ & $0.465(1)$ & $0.6686(8)$ & $0.1636(8)$ & $3.0(3)$ \\
\hline $\mathrm{C}(84)$ & $0.4877(12)$ & $0.7024(7)$ & $0.2030(7)$ & $2.2(3)$ \\
\hline $\mathrm{H}(1)$ & $-0.1580(13)$ & $0.6925(8)$ & $0.7396(8)$ & $3.5(4)$ \\
\hline $\mathrm{H}(2)$ & $-0.221(2)$ & $0.7048(9)$ & $0.6446(8)$ & $4.3(4)$ \\
\hline $\mathrm{H}(3)$ & $-0.332(2)$ & $0.8081(10)$ & $0.5877(8)$ & $5.1(5)$ \\
\hline $\mathrm{H}(4)$ & $-0.394(2)$ & $0.9010(9)$ & $0.6380(8)$ & $4.1(4)$ \\
\hline $\mathrm{H}(5)$ & $-0.3186(12)$ & $0.8878(7)$ & $0.7318(7)$ & $2.8(3)$ \\
\hline $\mathrm{H}(6)$ & $-0.4313(12)$ & $0.7995(7)$ & $0.8504(7)$ & $2.7(3)$ \\
\hline $\mathrm{H}(7)$ & $-0.5837(12)$ & $0.8150(7)$ & $0.9414(8)$ & $2.8(3)$ \\
\hline
\end{tabular}




\begin{tabular}{|c|c|c|c|c|}
\hline $\mathrm{H}(8)$ & $-0.5480(12)$ & $0.8354(8)$ & $1.0325(8)$ & $3.0(3)$ \\
\hline $\mathrm{H}(9)$ & $-0.349(1)$ & $0.8345(8)$ & $1.0343(8)$ & $3.7(4)$ \\
\hline $\mathrm{H}(10)$ & $-0.1959(11)$ & $0.8159(7)$ & $0.9442(7)$ & $2.4(3)$ \\
\hline $\mathrm{H}(11)$ & $0.2178(13)$ & $0.5603(7)$ & $0.8442(8)$ & $3.2(4)$ \\
\hline $\mathrm{H}(12)$ & $0.391(1)$ & $0.5035(9)$ & $0.7930(9)$ & $4.4(5)$ \\
\hline $\mathrm{H}(13)$ & $0.557(2)$ & $0.5671(10)$ & $0.7302(10)$ & $5.2(5)$ \\
\hline $\mathrm{H}(14)$ & $0.548(2)$ & $0.6871(9)$ & $0.7241(11)$ & $4.9(5)$ \\
\hline $\mathrm{H}(15)$ & $0.3734(13)$ & $0.7423(8)$ & $0.7745(9)$ & $3.6(4)$ \\
\hline $\mathrm{H}(16)$ & $0.0162(12)$ & $0.7332(7)$ & $0.9835(7)$ & $2.8(3)$ \\
\hline $\mathrm{H}(17)$ & $0.063(1)$ & $0.7360(8)$ & $1.0811(9)$ & $3.9(4)$ \\
\hline $\mathrm{H}(18)$ & $0.2577(11)$ & $0.6954(6)$ & $1.0981(7)$ & $2.5(3)$ \\
\hline $\mathrm{H}(19)$ & $0.4005(13)$ & $0.6574(8)$ & $1.0163(9)$ & $3.8(4)$ \\
\hline $\mathrm{H}(20)$ & $0.3533(12)$ & $0.6574(7)$ & $0.9192(8)$ & $2.7(3)$ \\
\hline $\mathrm{H}(21)$ & $-0.2163(13)$ & $0.4778(7)$ & $0.9875(8)$ & $3.2(4)$ \\
\hline $\mathrm{H}(22)$ & $-0.0990(13)$ & $0.4973(7)$ & $0.9902(8)$ & $3.2(4)$ \\
\hline $\mathrm{H}(23)$ & $-0.134(2)$ & $0.4880(8)$ & $0.8705(11)$ & $5.1(5)$ \\
\hline $\mathrm{H}(24)$ & $-0.017(2)$ & $0.5078(8)$ & $0.8731(11)$ & $5.1(5)$ \\
\hline $\mathrm{H}(25)$ & $-0.055(2)$ & $0.4305(8)$ & $0.9178(11)$ & $5.1(5)$ \\
\hline $\mathrm{H}(26)$ & $-0.2713(12)$ & $0.5971(7)$ & $0.8412(7)$ & $2.5(3)$ \\
\hline $\mathrm{H}(27)$ & $-0.3533(12)$ & $0.6521(7)$ & $0.8716(7)$ & $2.5(3)$ \\
\hline $\mathrm{H}(28)$ & $-0.4566(13)$ & $0.5591(8)$ & $0.8903(9)$ & $3.5(4)$ \\
\hline $\mathrm{H}(29)$ & $-0.4527(13)$ & $0.5592(8)$ & $0.9621(9)$ & $3.5(4)$ \\
\hline $\mathrm{H}(30)$ & $-0.3696(13)$ & $0.5035(8)$ & $0.9332(9)$ & $3.5(4)$ \\
\hline $\mathrm{H}(31)$ & $-0.344(1)$ & $0.6579(8)$ & $0.9975(8)$ & $3.6(4)$ \\
\hline $\mathrm{H}(32)$ & $-0.345(1)$ & $0.5747(8)$ & $1.0362(8)$ & $3.6(4)$ \\
\hline $\mathrm{H}(33)$ & $-0.271(2)$ & $0.6211(9)$ & $1.0983(9)$ & $5.1(5)$ \\
\hline $\mathrm{H}(34)$ & $-0.173(2)$ & $0.5691(9)$ & $1.0713(9)$ & $5.1(5)$ \\
\hline $\mathrm{H}(35)$ & $-0.171(2)$ & $0.6523(9)$ & $1.0320(9)$ & $5.1(5)$ \\
\hline $\mathrm{H}(36)$ & $0.3143(13)$ & $0.8414(8)$ & $0.6466(8)$ & $3.5(4)$ \\
\hline $\mathrm{H}(37)$ & $0.2303(13)$ & $0.9125(8)$ & $0.6353(8)$ & $3.5(4)$ \\
\hline $\mathrm{H}(38)$ & $0.224(2)$ & $0.9159(8)$ & $0.5269(9)$ & $4.4(5)$ \\
\hline $\mathrm{H}(39)$ & $0.354(2)$ & $0.9146(8)$ & $0.5279(9)$ & $4.4(5)$ \\
\hline $\mathrm{H}(40)$ & $0.307(2)$ & $0.8445(8)$ & $0.5382(9)$ & $4.4(5)$ \\
\hline $\mathrm{H}(41)$ & $0.2536(13)$ & $0.7137(8)$ & $0.6781(8)$ & $3.2(4)$ \\
\hline $\mathrm{H}(42)$ & $0.2083(13)$ & $0.7445(8)$ & $0.6093(8)$ & $3.2(4)$ \\
\hline $\mathrm{H}(43)$ & $0.0360(13)$ & $0.6891(7)$ & $0.6711(8)$ & $3.6(4)$ \\
\hline $\mathrm{H}(44)$ & $0.0799(13)$ & $0.6590(7)$ & $0.7404(8)$ & $3.6(4)$ \\
\hline $\mathrm{H}(45)$ & $0.1436(13)$ & $0.6309(7)$ & $0.6804(8)$ & $3.7(4)$ \\
\hline $\mathrm{H}(46)$ & $0.0660(13)$ & $0.8570(7)$ & $0.5702(7)$ & $2.7(3)$ \\
\hline $\mathrm{H}(47)$ & $-0.0409(13)$ & $0.8284(7)$ & $0.6322(7)$ & $2.7(3)$ \\
\hline $\mathrm{H}(48)$ & $0.033(2)$ & $0.9660(8)$ & $0.5870(9)$ & $3.9(4)$ \\
\hline $\mathrm{H}(49)$ & $-0.080(2)$ & $0.9519(8)$ & $0.5740(9)$ & $3.9(4)$ \\
\hline $\mathrm{H}(50)$ & $-0.072(2)$ & $0.9374(8)$ & $0.6500(9)$ & $3.9(4)$ \\
\hline $\mathrm{H}(51)$ & $0.1298(11)$ & $0.9847(6)$ & $0.7135(7)$ & $2.4(3)$ \\
\hline $\mathrm{H}(52)$ & $0.1198(11)$ & $1.0132(6)$ & $0.7722(7)$ & $2.4(3)$ \\
\hline
\end{tabular}




\begin{tabular}{|c|c|c|c|c|}
\hline $\mathrm{H}(53)$ & $-0.0727(13)$ & $0.9907(7)$ & $0.7377(9)$ & $3.6(4)$ \\
\hline $\mathrm{H}(54)$ & $-0.0339(13)$ & $1.0666(7)$ & $0.7165(9)$ & $3.6(4)$ \\
\hline $\mathrm{H}(55)$ & $-0.0818(13)$ & $1.0205(7)$ & $0.7953(9)$ & $3.6(4)$ \\
\hline $\mathrm{H}(56)$ & $0.2554(12)$ & $0.8342(7)$ & $0.8699(8)$ & $3.1(4)$ \\
\hline $\mathrm{H}(57)$ & $0.2412(12)$ & $0.9159(7)$ & $0.8568(8)$ & $3.1(4)$ \\
\hline $\mathrm{H}(58)$ & $0.4127(13)$ & $0.8893(8)$ & $0.7820(9)$ & $4.1(4)$ \\
\hline $\mathrm{H}(59)$ & $0.3248(13)$ & $0.9418(8)$ & $0.7378(9)$ & $4.1(4)$ \\
\hline $\mathrm{H}(60)$ & $0.3399(13)$ & $0.8600(8)$ & $0.7512(9)$ & $4.1(4)$ \\
\hline $\mathrm{H}(61)$ & $-0.0804(13)$ & $0.9185(7)$ & $0.8955(8)$ & $3.2(4)$ \\
\hline $\mathrm{H}(62)$ & $-0.0029(13)$ & $0.8567(7)$ & $0.9408(8)$ & $3.2(4)$ \\
\hline $\mathrm{H}(63)$ & $0.023(2)$ & $1.0018(10)$ & $0.8955(10)$ & $6.4(6)$ \\
\hline $\mathrm{H}(64)$ & $0.097(2)$ & $0.9394(10)$ & $0.9427(10)$ & $6.4(6)$ \\
\hline $\mathrm{H}(65)$ & $-0.037(2)$ & $0.9551(10)$ & $0.9720(10)$ & $6.4(6)$ \\
\hline $\mathrm{H}(66)$ & $0.891(1)$ & $0.8777(8)$ & $0.1676(8)$ & $3.6(4)$ \\
\hline $\mathrm{H}(67)$ & $1.081(2)$ & $0.8743(9)$ & $0.0965(10)$ & $4.8(5)$ \\
\hline $\mathrm{H}(68)$ & $1.234(2)$ & $0.7999(12)$ & $0.1467(13)$ & $6.9(8)$ \\
\hline $\mathrm{H}(69)$ & $1.197(2)$ & $0.7286(11)$ & $0.2649(12)$ & $6.3(6)$ \\
\hline $\mathrm{H}(70)$ & $1.004(1)$ & $0.7277(9)$ & $0.3335(10)$ & $4.4(5)$ \\
\hline $\mathrm{H}(71)$ & $0.929(1)$ & $0.8214(8)$ & $0.3869(9)$ & $3.7(4)$ \\
\hline $\mathrm{H}(72)$ & $0.914(2)$ & $0.8835(8)$ & $0.4620(8)$ & $4.3(4)$ \\
\hline $\mathrm{H}(73)$ & $0.736(2)$ & $0.9523(8)$ & $0.4886(9)$ & $4.6(5)$ \\
\hline $\mathrm{H}(74)$ & $0.580(2)$ & $0.9574(8)$ & $0.4439(9)$ & $4.6(5)$ \\
\hline $\mathrm{H}(75)$ & $0.597(2)$ & $0.8944(9)$ & $0.3705(9)$ & $4.2(4)$ \\
\hline $\mathrm{H}(76)$ & $0.276(2)$ & $0.8193(11)$ & $0.3306(10)$ & $4.9(5)$ \\
\hline $\mathrm{H}(77)$ & $0.098(2)$ & $0.8291(13)$ & $0.4077(12)$ & $6.7(7)$ \\
\hline $\mathrm{H}(78)$ & $0.051(2)$ & $0.7389(12)$ & $0.5207(11)$ & $6.1(6)$ \\
\hline $\mathrm{H}(79)$ & $0.189(2)$ & $0.6434(11)$ & $0.5557(10)$ & $5.4(5)$ \\
\hline $\mathrm{H}(80)$ & $0.374(2)$ & $0.6356(9)$ & $0.4791(9)$ & $4.6(5)$ \\
\hline $\mathrm{H}(81)$ & $0.347(2)$ & $0.6061(8)$ & $0.3578(9)$ & $4.4(5)$ \\
\hline $\mathrm{H}(82)$ & $0.312(2)$ & $0.5458(9)$ & $0.2917(9)$ & $5.4(5)$ \\
\hline $\mathrm{H}(83)$ & $0.386(1)$ & $0.5849(7)$ & $0.1709(8)$ & $4.1(4)$ \\
\hline $\mathrm{H}(84)$ & $0.493(1)$ & $0.6863(8)$ & $0.1139(8)$ & $3.7(4)$ \\
\hline $\mathrm{H}(85)$ & $0.5315(12)$ & $0.7433(7)$ & $0.1793(7)$ & $2.9(3)$ \\
\hline $\mathrm{H}(86)$ & $0.281(2)$ & $0.9370(10)$ & $0.2724(10)$ & $5.1(5)$ \\
\hline $\mathrm{H}(87)$ & $0.337(2)$ & $1.0096(10)$ & $0.2308(10)$ & $5.1(5)$ \\
\hline $\mathrm{H}(88)$ & $0.388(2)$ & $0.9071(9)$ & $0.3557(10)$ & $5.0(5)$ \\
\hline $\mathrm{H}(89)$ & $0.446(2)$ & $0.9792(9)$ & $0.3138(10)$ & $5.0(5)$ \\
\hline $\mathrm{H}(90)$ & $0.312(2)$ & $0.9803(9)$ & $0.3492(10)$ & $5.0(5)$ \\
\hline $\mathrm{H}(91)$ & $0.591(2)$ & $1.0088(8)$ & $0.1733(10)$ & $5.2(5)$ \\
\hline $\mathrm{H}(92)$ & $0.506(2)$ & $1.0299(8)$ & $0.1234(10)$ & $5.2(5)$ \\
\hline $\mathrm{H}(93)$ & $0.699(2)$ & $1.0136(11)$ & $0.0626(10)$ & $5.5(5)$ \\
\hline $\mathrm{H}(94)$ & $0.626(2)$ & $0.9569(11)$ & $0.0666(10)$ & $5.5(5)$ \\
\hline $\mathrm{H}(95)$ & $0.710(2)$ & $0.9349(11)$ & $0.1169(10)$ & $5.5(5)$ \\
\hline $\mathrm{H}(96)$ & $0.345(1)$ & $0.8654(8)$ & $0.1828(8)$ & $3.5(4)$ \\
\hline $\mathrm{H}(97)$ & $0.460(1)$ & $0.8776(8)$ & $0.1239(8)$ & $3.5(4)$ \\
\hline
\end{tabular}




$\begin{array}{lllll}\mathrm{H}(98) & 0.280(2) & 0.9860(9) & 0.1339(8) & 4.5(5) \\ \mathrm{H}(99) & 0.395(2) & 0.9974(9) & 0.0745(8) & 4.5(5) \\ \mathrm{H}(100) & 0.304(2) & 0.9501(9) & 0.0792(8) & 4.5(5) \\ \mathrm{H}(101) & 0.688(2) & 0.7500(9) & 0.5021(9) & 4.7(5) \\ \mathrm{H}(102) & 0.657(2) & 0.6813(9) & 0.5692(9) & 4.7(5) \\ \mathrm{H}(103) & 0.505(2) & 0.762(1) & 0.4828(12) & 8.2(8) \\ \mathrm{H}(104) & 0.474(2) & 0.693(1) & 0.5490(12) & 8.2(8) \\ \mathrm{H}(105) & 0.493(2) & 0.761(1) & 0.5572(12) & 8.2(8) \\ \mathrm{H}(106) & 0.657(5) & 0.519(1) & 0.467(2) & 23.0(30) \\ \mathrm{H}(107) & 0.659(3) & 0.5571(12) & 0.5762(11) & 11.9(14) \\ \mathrm{H}(108) & 0.637(5) & 0.467(1) & 0.546(2) & 23.0(30) \\ \mathrm{H}(109) & 0.9414(13) & 0.7014(8) & 0.1988(8) & 3.6(4) \\ \mathrm{H}(110) & 0.758(5) & 0.497(1) & 0.506(2) & 23.0(30) \\ \mathrm{H}(111) & 0.897(2) & 0.6064(11) & 0.4520(10) & 6.6(7) \\ \mathrm{H}(112) & 0.887(2) & 0.6783(11) & 0.4629(10) & 6.6(7) \\ \mathrm{H}(113) & 0.965(3) & 0.586(1) & 0.5465(13) & 10.7(12) \\ \mathrm{H}(114) & 0.845(3) & 0.619(1) & 0.5790(13) & 10.7(12) \\ \mathrm{H}(115) & 0.852(3) & 0.547(1) & 0.5688(13) & 10.7(12) \\ \mathrm{H}(116) & 0.900(2) & 0.5353(8) & 0.3673(9) & 4.5(5) \\ \mathrm{H}(117) & 0.976(2) & 0.5998(8) & 0.3273(9) & 4.5(5) \\ \mathrm{H}(118) & 1.050(2) & 0.5610(10) & 0.2355(10) & 6.1(7) \\ \mathrm{H}(119) & 1.074(2) & 0.4991(10) & 0.3043(10) & 6.1(7) \\ \mathrm{H}(120) & 0.972(2) & 0.4973(10) & 0.2741(10) & 6.1(7) \\ \mathrm{H}(121) & 0.673(2) & 0.5940(7) & 0.2478(8) & 3.7(4) \\ \mathrm{H}(122) & 0.790(2) & 0.5443(7) & 0.2440(8) & 3.7(4) \\ \mathrm{H}(123) & 0.616(2) & 0.5271(9) & 0.3674(9) & 5.1(5) \\ \mathrm{H}(124) & 0.639(2) & 0.4774(9) & 0.3252(9) & 5.1(5) \\ \mathrm{H}(125) & 0.733(2) & 0.4768(9) & 0.3626(9) & 5.1(5) \\ \mathrm{H}(126) & 0.556(3) & 0.5789(12) & 0.5378(11) & 11.9(14) \\ \mathrm{H}(127) & 0.9162(13) & 0.6446(8) & 0.1749(8) & 3.6(4) \\ \mathrm{H}(128) & 0.7666(13) & 0.7704(8) & 0.1740(8) & 3.3(4) \\ \mathrm{H}(129) & 0.8459(13) & 0.7600(8) & 0.1063(8) & 3.3(4) \\ \mathrm{H}(130) & 0.7411(13) & 0.7136(8) & 0.1503(8) & 3.3(4) \\ \mathrm{H}(131) & 0.567(9) & 0.691(6) & 0.369(5) & 5.9(19) \\ \mathrm{H}(132) & 0.72(1) & 0.840(9) & 0.267(9) & -0.3(38)\end{array}$

$\mathrm{B}_{\mathrm{eq}}=8 / 3 \pi^{2}\left(\mathrm{U}_{11}\left(\mathrm{aa}^{*}\right)^{2}+\mathrm{U}_{22}\left(\mathrm{bb}^{*}\right)^{2}+\mathrm{U}_{33}(\mathrm{cc} *)^{2}+2 \mathrm{U}_{12}\left(\mathrm{aa}^{*} \mathrm{bb}^{*}\right) \cos \gamma+2 \mathrm{U}_{13}\left(\mathrm{aa}^{*} \mathrm{cc} *\right) \cos \beta+\right.$ $\left.2 \mathrm{U}_{23}\left(\mathrm{bb}^{*} \mathrm{cc} *\right) \cos \alpha\right)$

Table 2. Anisotropic Displacement Parameters

$\begin{array}{llllccc}\text { atom } & \mathrm{U}_{11} & \mathrm{U}_{22} & \mathrm{U}_{33} & \mathrm{U}_{12} & \mathrm{U} 13 & \mathrm{U}_{23} \\ \mathrm{Pt}(1) & 0.0208(2) & 0.0131(2) & 0.0275(3) & -0.0037(2) & -0.0037(2) & -0.0085(2) \\ \operatorname{Pt}(2) & 0.0400(3) & 0.0164(2) & 0.0250(3) & -0.0025(2) & -0.0104(2) & -0.0089(2) \\ \operatorname{Pd}(1) & 0.0124(4) & 0.0053(3) & 0.0189(4) & -0.0036(3) & -0.0006(3) & -0.0053(3)\end{array}$




\begin{tabular}{|c|c|c|c|c|c|c|}
\hline $\operatorname{Pd}(2)$ & $0.0271(5)$ & $0.0139(4)$ & $0.0238(5)$ & $-0.0008(3)$ & $-0.0079(4)$ & $-0.0073(4)$ \\
\hline $\mathrm{P}(1)$ & $0.021(2)$ & $0.015(1)$ & $0.028(2)$ & $-0.0089(11)$ & $-0.0009(13)$ & $-0.0064(12)$ \\
\hline $\mathrm{P}(2)$ & $0.024(2)$ & $0.020(2)$ & $0.029(2)$ & $-0.0043(12)$ & $0.004(1)$ & $-0.0124(13)$ \\
\hline $\mathrm{P}(3)$ & $0.026(2)$ & $0.0110(13)$ & $0.036(2)$ & $-0.0051(11)$ & $-0.010(1)$ & $-0.0087(13)$ \\
\hline $\mathrm{P}(4)$ & $0.036(2)$ & $0.020(2)$ & $0.035(2)$ & $0.003(1)$ & $-0.013(2)$ & $-0.010(1)$ \\
\hline $\mathrm{P}(5)$ & $0.071(3)$ & $0.025(2)$ & $0.028(2)$ & $-0.009(2)$ & $-0.019(2)$ & $-0.008(2)$ \\
\hline$P(6)$ & $0.046(2)$ & $0.020(2)$ & $0.030(2)$ & $0.003(1)$ & $-0.014(2)$ & $-0.014(1)$ \\
\hline $\mathrm{Si}(1)$ & $0.018(2)$ & $0.016(1)$ & $0.021(2)$ & $-0.0034(12)$ & $-0.0040(13)$ & $-0.0079(12)$ \\
\hline $\operatorname{Si}(2)$ & $0.016(2)$ & $0.017(2)$ & $0.033(2)$ & $0.0005(12)$ & $-0.003(1)$ & $-0.014(1)$ \\
\hline $\operatorname{Si}(3)$ & $0.028(2)$ & $0.021(2)$ & $0.037(2)$ & $-0.002(1)$ & $-0.009(2)$ & $-0.016(2)$ \\
\hline $\mathrm{Si}(4)$ & $0.037(2)$ & $0.024(2)$ & $0.027(2)$ & $-0.009(2)$ & $-0.007(2)$ & $-0.011(2)$ \\
\hline $\mathrm{C}(1)$ & $0.028(7)$ & $0.034(7)$ & $0.016(6)$ & $-0.016(5)$ & $-0.000(5)$ & $-0.004(5)$ \\
\hline $\mathrm{C}(2)$ & $0.039(8)$ & $0.037(8)$ & $0.035(8)$ & $-0.018(6)$ & $-0.002(6)$ & $-0.018(6)$ \\
\hline $\mathrm{C}(3)$ & $0.061(11)$ & $0.044(9)$ & $0.032(8)$ & $-0.030(8)$ & $-0.005(7)$ & $-0.015(7)$ \\
\hline $\mathrm{C}(4)$ & $0.074(12)$ & $0.065(11)$ & $0.024(7)$ & $-0.043(10)$ & $-0.017(8)$ & $-0.003(7)$ \\
\hline $\mathrm{C}(5)$ & $0.059(11)$ & $0.035(8)$ & $0.037(8)$ & $-0.009(7)$ & $-0.024(8)$ & $-0.002(7)$ \\
\hline $\mathrm{C}(6)$ & $0.033(7)$ & $0.028(7)$ & $0.029(7)$ & $0.003(5)$ & $-0.014(6)$ & $-0.013(5)$ \\
\hline $\mathrm{C}(7)$ & $0.017(6)$ & $0.018(5)$ & $0.023(6)$ & $-0.004(4)$ & $-0.000(5)$ & $-0.008(5)$ \\
\hline $\mathrm{C}(8)$ & $0.031(7)$ & $0.025(6)$ & $0.030(7)$ & $0.002(5)$ & $-0.015(6)$ & $-0.012(5)$ \\
\hline $\mathrm{C}(9)$ & $0.018(6)$ & $0.027(7)$ & $0.044(8)$ & $-0.001(5)$ & $-0.003(6)$ & $-0.006(6)$ \\
\hline$C(10)$ & $0.022(7)$ & $0.037(8)$ & $0.035(8)$ & $-0.007(6)$ & $0.000(6)$ & $-0.013(6)$ \\
\hline$C(11)$ & $0.052(10)$ & $0.032(7)$ & $0.033(8)$ & $-0.001(7)$ & $-0.011(7)$ & $-0.018(6)$ \\
\hline$C(12)$ & $0.019(6)$ & $0.034(7)$ & $0.022(6)$ & $-0.003(5)$ & $0.000(5)$ & $-0.013(5)$ \\
\hline$C(13)$ & $0.022(6)$ & $0.020(6)$ & $0.038(7)$ & $-0.000(5)$ & $-0.006(6)$ & $-0.013(5)$ \\
\hline $\mathrm{C}(14)$ & $0.035(8)$ & $0.024(7)$ & $0.043(8)$ & $-0.007(6)$ & $-0.006(7)$ & $-0.015(6)$ \\
\hline$C(15)$ & $0.044(9)$ & $0.041(9)$ & $0.055(10)$ & $-0.001(7)$ & $-0.007(8)$ & $-0.031(8)$ \\
\hline$C(16)$ & $0.042(10)$ & $0.059(11)$ & $0.064(12)$ & $0.018(8)$ & $-0.001(9)$ & $-0.042(10)$ \\
\hline$C(17)$ & $0.031(9)$ & $0.042(9)$ & $0.08(1)$ & $-0.011(7)$ & $0.001(9)$ & $-0.022(9)$ \\
\hline $\mathrm{C}(18)$ & $0.030(8)$ & $0.027(7)$ & $0.056(10)$ & $-0.004(6)$ & $-0.000(7)$ & $-0.014(7)$ \\
\hline$C(19)$ & $0.015(6)$ & $0.013(5)$ & $0.031(7)$ & $-0.002(4)$ & $-0.002(5)$ & $-0.004(5)$ \\
\hline$C(20)$ & $0.024(7)$ & $0.025(6)$ & $0.040(8)$ & $-0.004(5)$ & $-0.011(6)$ & $-0.016(6)$ \\
\hline $\mathrm{C}(21)$ & $0.038(9)$ & $0.037(8)$ & $0.050(10)$ & $-0.001(7)$ & $-0.007(7)$ & $-0.023(7)$ \\
\hline$C(22)$ & $0.029(7)$ & $0.012(5)$ & $0.036(7)$ & $-0.001(5)$ & $-0.014(6)$ & $-0.012(5)$ \\
\hline $\mathrm{C}(23)$ & $0.029(8)$ & $0.028(7)$ & $0.063(10)$ & $0.006(6)$ & $-0.021(7)$ & $-0.020(7)$ \\
\hline $\mathrm{C}(24)$ & $0.024(7)$ & $0.022(6)$ & $0.039(8)$ & $0.005(5)$ & $-0.015(6)$ & $-0.012(6)$ \\
\hline$C(25)$ & $0.033(8)$ & $0.016(6)$ & $0.052(9)$ & $-0.012(5)$ & $-0.010(7)$ & $-0.001(6)$ \\
\hline$C(26)$ & $0.058(11)$ & $0.016(7)$ & $0.09(1)$ & $-0.002(7)$ & $0.004(10)$ & $-0.028(8)$ \\
\hline $\mathrm{C}(27)$ & $0.031(7)$ & $0.018(6)$ & $0.031(7)$ & $-0.009(5)$ & $-0.012(6)$ & $-0.005(5)$ \\
\hline $\mathrm{C}(28)$ & $0.031(8)$ & $0.029(7)$ & $0.052(9)$ & $-0.015(6)$ & $-0.003(7)$ & $-0.014(7)$ \\
\hline $\mathrm{C}(29)$ & $0.042(9)$ & $0.036(8)$ & $0.035(8)$ & $-0.021(7)$ & $0.012(7)$ & $-0.020(7)$ \\
\hline $\mathrm{C}(30)$ & $0.075(13)$ & $0.041(9)$ & $0.046(10)$ & $-0.022(9)$ & $0.003(9)$ & $-0.025(8)$ \\
\hline $\mathrm{C}(31)$ & $0.032(8)$ & $0.031(7)$ & $0.048(9)$ & $-0.013(6)$ & $0.009(7)$ & $-0.020(7)$ \\
\hline$C(32)$ & $0.048(10)$ & $0.030(8)$ & $0.061(11)$ & $-0.014(7)$ & $0.014(8)$ & $-0.023(8)$ \\
\hline $\mathrm{C}(33)$ & $0.033(8)$ & $0.035(8)$ & $0.033(8)$ & $-0.007(6)$ & $0.003(6)$ & $-0.014(6)$ \\
\hline $\mathrm{C}(34)$ & $0.040(8)$ & $0.025(7)$ & $0.050(9)$ & $-0.007(6)$ & $-0.007(7)$ & $-0.025(7)$ \\
\hline
\end{tabular}




\begin{tabular}{|c|c|c|c|c|c|c|}
\hline $\mathrm{C}(35)$ & $0.036(8)$ & $0.030(7)$ & $0.020(6)$ & $-0.008(6)$ & $0.004(6)$ & $-0.012(5)$ \\
\hline$C(36)$ & $0.061(11)$ & $0.026(7)$ & $0.038(9)$ & $-0.004(7)$ & $-0.008(8)$ & $-0.006(6)$ \\
\hline $\mathrm{C}(37)$ & $0.025(7)$ & $0.017(6)$ & $0.035(7)$ & $0.000(5)$ & $-0.004(6)$ & $-0.010(5)$ \\
\hline $\mathrm{C}(38)$ & $0.038(8)$ & $0.017(6)$ & $0.060(10)$ & $0.005(6)$ & $-0.018(8)$ & $-0.014(6)$ \\
\hline $\mathrm{C}(39)$ & $0.029(7)$ & $0.020(6)$ & $0.048(8)$ & $-0.006(5)$ & $-0.016(6)$ & $-0.011(6)$ \\
\hline$C(40)$ & $0.035(8)$ & $0.034(8)$ & $0.062(10)$ & $-0.007(6)$ & $-0.014(7)$ & $-0.022(8)$ \\
\hline $\mathrm{C}(41)$ & $0.042(8)$ & $0.021(6)$ & $0.038(8)$ & $-0.005(6)$ & $-0.011(7)$ & $-0.011(6)$ \\
\hline $\mathrm{C}(42)$ & $0.09(2)$ & $0.057(11)$ & $0.060(12)$ & $-0.026(10)$ & $-0.011(10)$ & $-0.039(10)$ \\
\hline $\mathrm{C}(43)$ & $0.046(9)$ & $0.022(6)$ & $0.047(9)$ & $-0.000(6)$ & $-0.013(7)$ & $-0.022(6)$ \\
\hline$C(44)$ & $0.040(9)$ & $0.031(7)$ & $0.044(9)$ & $-0.016(6)$ & $-0.002(7)$ & $-0.017(7)$ \\
\hline$C(45)$ & $0.053(11)$ & $0.035(8)$ & $0.064(11)$ & $-0.012(7)$ & $0.005(9)$ & $-0.035(8)$ \\
\hline$C(46)$ & $0.032(9)$ & $0.08(1)$ & $0.10(2)$ & $-0.016(9)$ & $-0.005(10)$ & $-0.07(1)$ \\
\hline $\mathrm{C}(47)$ & $0.053(11)$ & $0.068(13)$ & $0.08(1)$ & $-0.001(9)$ & $-0.026(10)$ & $-0.050(12)$ \\
\hline $\mathrm{C}(48)$ & $0.041(9)$ & $0.045(9)$ & $0.054(10)$ & $0.010(7)$ & $-0.022(8)$ & $-0.025(8)$ \\
\hline$C(49)$ & $0.041(8)$ & $0.015(6)$ & $0.028(7)$ & $-0.008(5)$ & $-0.005(6)$ & $-0.008(5)$ \\
\hline$C(50)$ & $0.045(9)$ & $0.045(9)$ & $0.044(9)$ & $-0.008(7)$ & $-0.011(7)$ & $-0.021(8)$ \\
\hline $\mathrm{C}(51)$ & $0.054(10)$ & $0.034(8)$ & $0.059(10)$ & $-0.000(7)$ & $-0.007(9)$ & $-0.032(8)$ \\
\hline$C(52)$ & $0.070(12)$ & $0.032(8)$ & $0.043(9)$ & $-0.022(8)$ & $-0.002(8)$ & $-0.017(7)$ \\
\hline$C(53)$ & $0.048(9)$ & $0.042(8)$ & $0.044(9)$ & $-0.018(7)$ & $-0.011(7)$ & $-0.021(7)$ \\
\hline$C(54)$ & $0.036(8)$ & $0.031(7)$ & $0.050(9)$ & $-0.002(6)$ & $-0.005(7)$ & $-0.021(7)$ \\
\hline$C(55)$ & $0.039(8)$ & $0.043(8)$ & $0.027(7)$ & $-0.012(6)$ & $-0.004(6)$ & $-0.019(6)$ \\
\hline$C(56)$ & $0.048(11)$ & $0.066(12)$ & $0.043(10)$ & $0.006(9)$ & $0.006(8)$ & $-0.021(9)$ \\
\hline$C(57)$ & $0.07(2)$ & $0.08(2)$ & $0.06(1)$ & $0.011(12)$ & $-0.012(11)$ & $-0.032(12)$ \\
\hline $\mathrm{C}(58)$ & $0.047(11)$ & $0.09(2)$ & $0.057(12)$ & $-0.011(10)$ & $-0.002(9)$ & $-0.050(11)$ \\
\hline $\mathrm{C}(59)$ & $0.065(13)$ & $0.058(11)$ & $0.046(10)$ & $-0.008(10)$ & $0.017(9)$ & $-0.035(9)$ \\
\hline$C(60)$ & $0.061(12)$ & $0.042(9)$ & $0.042(9)$ & $0.001(8)$ & $-0.008(8)$ & $-0.018(8)$ \\
\hline $\mathrm{C}(61)$ & $0.050(11)$ & $0.046(10)$ & $0.064(12)$ & $0.018(8)$ & $-0.021(9)$ & $-0.033(9)$ \\
\hline $\mathrm{C}(62)$ & $0.059(12)$ & $0.045(10)$ & $0.055(11)$ & $0.019(8)$ & $-0.022(9)$ & $-0.029(8)$ \\
\hline $\mathrm{C}(63)$ & $0.073(12)$ & $0.021(7)$ & $0.072(12)$ & $-0.019(7)$ & $-0.034(10)$ & $-0.006(8)$ \\
\hline $\mathrm{C}(64)$ & $0.067(13)$ & $0.059(12)$ & $0.047(10)$ & $-0.030(10)$ & $-0.011(9)$ & $-0.005(9)$ \\
\hline $\mathrm{C}(65)$ & $0.045(9)$ & $0.034(7)$ & $0.033(7)$ & $-0.003(6)$ & $-0.026(7)$ & $-0.008(6)$ \\
\hline $\mathrm{C}(66)$ & $0.065(12)$ & $0.052(10)$ & $0.027(8)$ & $-0.003(8)$ & $-0.023(8)$ & $-0.005(7)$ \\
\hline $\mathrm{C}(67)$ & $0.066(12)$ & $0.039(9)$ & $0.043(9)$ & $-0.000(8)$ & $-0.015(8)$ & $-0.026(8)$ \\
\hline $\mathrm{C}(68)$ & $0.07(2)$ & $0.12(2)$ & $0.07(1)$ & $-0.03(1)$ & $-0.012(12)$ & $-0.05(2)$ \\
\hline $\mathrm{C}(69)$ & $0.09(2)$ & $0.063(12)$ & $0.053(11)$ & $0.029(11)$ & $-0.043(11)$ & $-0.034(10)$ \\
\hline $\mathrm{C}(70)$ & $0.16(3)$ & $0.10(2)$ & $0.08(2)$ & $0.05(2)$ & $-0.09(2)$ & $-0.05(2)$ \\
\hline $\mathrm{C}(71)$ & $0.28(4)$ & $0.053(13)$ & $0.045(12)$ & $-0.09(2)$ & $-0.07(2)$ & $0.026(10)$ \\
\hline $\mathrm{C}(72)$ & $0.61(9)$ & $0.05(2)$ & $0.07(2)$ & $-0.10(3)$ & $-0.13(4)$ & $0.010(13)$ \\
\hline $\mathrm{C}(73)$ & $0.061(11)$ & $0.032(8)$ & $0.048(9)$ & $0.024(7)$ & $-0.029(8)$ & $-0.024(7)$ \\
\hline $\mathrm{C}(74)$ & $0.077(13)$ & $0.051(11)$ & $0.066(12)$ & $0.030(10)$ & $-0.036(11)$ & $-0.043(10)$ \\
\hline$C(75)$ & $0.063(11)$ & $0.022(7)$ & $0.034(8)$ & $-0.004(6)$ & $-0.018(7)$ & $-0.009(6)$ \\
\hline$C(76)$ & $0.09(1)$ & $0.035(8)$ & $0.038(9)$ & $-0.016(9)$ & $-0.006(9)$ & $-0.015(7)$ \\
\hline $\mathrm{C}(77)$ & $0.039(8)$ & $0.036(8)$ & $0.041(8)$ & $-0.001(6)$ & $-0.009(7)$ & $-0.026(7)$ \\
\hline $\mathrm{C}(78)$ & $0.038(8)$ & $0.031(7)$ & $0.035(8)$ & $0.000(6)$ & $-0.014(7)$ & $-0.012(6)$ \\
\hline $\mathrm{C}(79)$ & $0.045(8)$ & $0.020(6)$ & $0.033(7)$ & $-0.005(6)$ & $-0.019(7)$ & $-0.006(5)$ \\
\hline
\end{tabular}




$\begin{array}{lllllll}\mathrm{C}(80) & 0.075(12) & 0.030(8) & 0.034(8) & -0.021(8) & -0.012(8) & -0.007(6) \\ \mathrm{C}(81) & 0.09(1) & 0.037(9) & 0.042(9) & -0.034(9) & -0.023(9) & -0.006(7) \\ \mathrm{C}(82) & 0.058(10) & 0.023(6) & 0.048(9) & -0.001(6) & -0.034(8) & -0.017(6) \\ \mathrm{C}(83) & 0.040(9) & 0.043(8) & 0.032(8) & 0.012(7) & -0.007(7) & -0.023(7) \\ \mathrm{C}(84) & 0.035(7) & 0.020(6) & 0.036(7) & -0.006(5) & -0.010(6) & -0.015(6)\end{array}$

The general temperature factor expression: $\exp \left(-2 \pi^{2}\left(a^{* 2} U_{11} h^{2}+b^{* 2} U_{22} k^{2}+c^{* 2} U_{33} 1^{2}+\right.\right.$ $\left.\left.2 \mathrm{a} * \mathrm{~b} * \mathrm{U}_{12} \mathrm{hk}+2 \mathrm{a} * \mathrm{c} * \mathrm{U}_{13} \mathrm{hl}+2 \mathrm{~b} * \mathrm{c} * \mathrm{U}_{23} \mathrm{kl}\right)\right)$

Table 3. Bond lengths $(\AA)$

\begin{tabular}{|c|c|c|c|c|c|}
\hline atom & atom & distance & atom & atom & distance \\
\hline $\operatorname{Pt}(1)$ & $\operatorname{Pd}(1)$ & $2.7570(10)$ & $\operatorname{Pt}(1)$ & $\mathrm{P}(2)$ & $2.383(3)$ \\
\hline $\operatorname{Pt}(1)$ & $\mathrm{P}(3)$ & $2.325(3)$ & $\operatorname{Pt}(1)$ & $\operatorname{Si}(1)$ & $2.415(3)$ \\
\hline $\operatorname{Pt}(1)$ & $\operatorname{Si}(2)$ & $2.361(3)$ & $\operatorname{Pt}(2)$ & $\operatorname{Pd}(2)$ & $2.7390(10)$ \\
\hline $\operatorname{Pt}(2)$ & $\mathrm{P}(5)$ & $2.298(4)$ & $\operatorname{Pt}(2)$ & $\mathrm{P}(6)$ & $2.368(4)$ \\
\hline $\operatorname{Pt}(2)$ & $\operatorname{Si}(3)$ & $2.346(4)$ & $\operatorname{Pt}(2)$ & $\mathrm{Si}(4)$ & $2.393(4)$ \\
\hline $\operatorname{Pd}(1)$ & $\mathrm{P}(1)$ & $2.281(3)$ & $\operatorname{Pd}(1)$ & $\operatorname{Si}(1)$ & $2.300(3)$ \\
\hline $\operatorname{Pd}(1)$ & $\operatorname{Si}(2)$ & $2.388(3)$ & $\operatorname{Pd}(2)$ & $\mathrm{P}(4)$ & $2.271(4)$ \\
\hline $\operatorname{Pd}(2)$ & $\operatorname{Si}(3)$ & $2.410(4)$ & $\operatorname{Pd}(2)$ & $\mathrm{Si}(4)$ & $2.321(4)$ \\
\hline $\mathrm{P}(1)$ & $\mathrm{C}(25)$ & $1.813(13)$ & $\mathrm{P}(1)$ & $\mathrm{C}(27)$ & $1.814(12)$ \\
\hline $\mathrm{P}(1)$ & $\mathrm{C}(29)$ & $1.85(1)$ & $\mathrm{P}(2)$ & $\mathrm{C}(31)$ & $1.84(1)$ \\
\hline $\mathrm{P}(2)$ & $\mathrm{C}(33)$ & $1.84(1)$ & $\mathrm{P}(2)$ & $\mathrm{C}(35)$ & $1.83(1)$ \\
\hline $\mathrm{P}(3)$ & $\mathrm{C}(37)$ & $1.846(12)$ & $\mathrm{P}(3)$ & $\mathrm{C}(39)$ & $1.830(13)$ \\
\hline $\mathrm{P}(3)$ & $\mathrm{C}(41)$ & $1.85(2)$ & $\mathrm{P}(4)$ & $\mathrm{C}(61)$ & $1.88(2)$ \\
\hline $\mathrm{P}(4)$ & $\mathrm{C}(63)$ & $1.84(2)$ & $\mathrm{P}(4)$ & $\mathrm{C}(65)$ & $1.841(13)$ \\
\hline $\mathrm{P}(5)$ & $\mathrm{C}(67)$ & $1.82(2)$ & $\mathrm{P}(5)$ & $\mathrm{C}(69)$ & $1.81(2)$ \\
\hline $\mathrm{P}(5)$ & $\mathrm{C}(71)$ & $1.82(2)$ & $\mathrm{P}(6)$ & $\mathrm{C}(73)$ & $1.85(2)$ \\
\hline$P(6)$ & $\mathrm{C}(75)$ & $1.85(1)$ & $\mathrm{P}(6)$ & $\mathrm{C}(77)$ & $1.84(2)$ \\
\hline $\operatorname{Si}(1)$ & $\mathrm{C}(1)$ & $1.865(13)$ & $\operatorname{Si}(1)$ & $\mathrm{C}(7)$ & $1.893(12)$ \\
\hline $\operatorname{Si}(2)$ & $\mathrm{C}(13)$ & $1.904(13)$ & $\operatorname{Si}(2)$ & $\mathrm{C}(19)$ & $1.896(13)$ \\
\hline $\operatorname{Si}(3)$ & $\mathrm{C}(43)$ & $1.92(2)$ & $\operatorname{Si}(3)$ & $\mathrm{C}(49)$ & $1.919(13)$ \\
\hline $\operatorname{Si}(4)$ & $\mathrm{C}(55)$ & $1.91(2)$ & $\operatorname{Si}(4)$ & $\mathrm{C}(79)$ & 1.91(1) \\
\hline $\mathrm{C}(1)$ & $\mathrm{C}(2)$ & $1.42(2)$ & $\mathrm{C}(1)$ & $C(6)$ & $1.38(2)$ \\
\hline $\mathrm{C}(2)$ & $\mathrm{C}(3)$ & $1.36(2)$ & $\mathrm{C}(3)$ & $\mathrm{C}(4)$ & $1.36(3)$ \\
\hline $\mathrm{C}(4)$ & $\mathrm{C}(5)$ & $1.45(3)$ & $C(5)$ & $C(6)$ & $1.39(2)$ \\
\hline$C(7)$ & $\mathrm{C}(8)$ & $1.41(2)$ & $C(7)$ & $\mathrm{C}(12)$ & $1.37(2)$ \\
\hline $\mathrm{C}(8)$ & $\mathrm{C}(9)$ & $1.39(2)$ & $\mathrm{C}(9)$ & $\mathrm{C}(10)$ & $1.37(2)$ \\
\hline$C(10)$ & $\mathrm{C}(11)$ & $1.41(2)$ & $\mathrm{C}(11)$ & $\mathrm{C}(12)$ & $1.40(2)$ \\
\hline$C(13)$ & $\mathrm{C}(14)$ & $1.37(2)$ & $C(13)$ & $\mathrm{C}(18)$ & $1.38(2)$ \\
\hline$C(14)$ & $\mathrm{C}(15)$ & $1.41(2)$ & $C(15)$ & $C(16)$ & $1.38(3)$ \\
\hline$C(16)$ & $\mathrm{C}(17)$ & $1.42(2)$ & $C(17)$ & $\mathrm{C}(18)$ & $1.39(2)$ \\
\hline C(19) & $\mathrm{C}(20)$ & $1.42(2)$ & $C(19)$ & $\mathrm{C}(24)$ & $1.39(2)$ \\
\hline$C(20)$ & $\mathrm{C}(21)$ & $1.38(2)$ & $\mathrm{C}(21)$ & $C(22)$ & $1.41(2)$ \\
\hline
\end{tabular}




$\begin{array}{llllll}\mathrm{C}(22) & \mathrm{C}(23) & 1.37(2) & \mathrm{C}(23) & \mathrm{C}(24) & 1.37(2) \\ \mathrm{C}(25) & \mathrm{C}(26) & 1.54(2) & \mathrm{C}(27) & \mathrm{C}(28) & 1.52(2) \\ \mathrm{C}(29) & \mathrm{C}(30) & 1.53(2) & \mathrm{C}(31) & \mathrm{C}(32) & 1.53(2) \\ \mathrm{C}(33) & \mathrm{C}(34) & 1.53(2) & \mathrm{C}(35) & \mathrm{C}(36) & 1.54(2) \\ \mathrm{C}(37) & \mathrm{C}(38) & 1.50(2) & \mathrm{C}(39) & \mathrm{C}(40) & 1.52(2) \\ \mathrm{C}(41) & \mathrm{C}(42) & 1.51(2) & \mathrm{C}(43) & \mathrm{C}(44) & 1.36(2) \\ \mathrm{C}(43) & \mathrm{C}(48) & 1.38(2) & \mathrm{C}(44) & \mathrm{C}(45) & 1.39(2) \\ \mathrm{C}(45) & \mathrm{C}(46) & 1.37(3) & \mathrm{C}(46) & \mathrm{C}(47) & 1.35(3) \\ \mathrm{C}(47) & \mathrm{C}(48) & 1.38(3) & \mathrm{C}(49) & \mathrm{C}(50) & 1.38(2) \\ \mathrm{C}(49) & \mathrm{C}(54) & 1.37(2) & \mathrm{C}(50) & \mathrm{C}(51) & 1.41(2) \\ \mathrm{C}(51) & \mathrm{C}(52) & 1.35(2) & \mathrm{C}(52) & \mathrm{C}(53) & 1.38(2) \\ \mathrm{C}(53) & \mathrm{C}(54) & 1.42(2) & \mathrm{C}(55) & \mathrm{C}(56) & 1.40(2) \\ \mathrm{C}(55) & \mathrm{C}(60) & 1.36(2) & \mathrm{C}(56) & \mathrm{C}(57) & 1.37(3) \\ \mathrm{C}(57) & \mathrm{C}(58) & 1.39(3) & \mathrm{C}(58) & \mathrm{C}(59) & 1.33(3) \\ \mathrm{C}(59) & \mathrm{C}(60) & 1.41(2) & \mathrm{C}(61) & \mathrm{C}(62) & 1.49(2) \\ \mathrm{C}(63) & \mathrm{C}(64) & 1.51(3) & \mathrm{C}(65) & \mathrm{C}(66) & 1.50(2) \\ \mathrm{C}(67) & \mathrm{C}(68) & 1.48(3) & \mathrm{C}(69) & \mathrm{C}(70) & 1.46(3) \\ \mathrm{C}(71) & \mathrm{C}(72) & 1.45(4) & \mathrm{C}(73) & \mathrm{C}(74) & 1.51(2) \\ \mathrm{C}(75) & \mathrm{C}(76) & 1.52(2) & \mathrm{C}(77) & \mathrm{C}(78) & 1.53(2) \\ \mathrm{C}(79) & \mathrm{C}(80) & 1.37(2) & \mathrm{C}(79) & \mathrm{C}(84) & 1.39(2) \\ \mathrm{C}(80) & \mathrm{C}(81) & 1.44(2) & \mathrm{C}(81) & \mathrm{C}(82) & 1.36(2) \\ \mathrm{C}(82) & \mathrm{C}(83) & 1.39(2) & \mathrm{C}(83) & \mathrm{C}(84) & 1.40(2)\end{array}$

Table 4. Bond lengths involving hydrogens $(\AA)$

$\begin{array}{llllll}\text { atom } & \text { atom } & \text { distance } & \text { atom } & \text { atom } & \text { distance } \\ \mathrm{Pt}(2) & \mathrm{H}(131) & 1.3(1) & \mathrm{Pd}(2) & \mathrm{H}(132) & 1.6(2) \\ \mathrm{Si}(3) & \mathrm{H}(132) & 1.2(3) & \mathrm{Si}(4) & \mathrm{H}(131) & 1.3(2) \\ \mathrm{C}(2) & \mathrm{H}(1) & 0.95(4) & \mathrm{C}(3) & \mathrm{H}(2) & 0.95(4) \\ \mathrm{C}(4) & \mathrm{H}(3) & 0.95(3) & \mathrm{C}(5) & \mathrm{H}(4) & 0.95(5) \\ \mathrm{C}(6) & \mathrm{H}(5) & 0.95(3) & \mathrm{C}(8) & \mathrm{H}(6) & 0.95(3) \\ \mathrm{C}(9) & \mathrm{H}(7) & 0.95(2) & \mathrm{C}(10) & \mathrm{H}(8) & 0.95(4) \\ \mathrm{C}(11) & \mathrm{H}(9) & 0.95(3) & \mathrm{C}(12) & \mathrm{H}(10) & 0.95(2) \\ \mathrm{C}(14) & \mathrm{H}(11) & 0.95(4) & \mathrm{C}(15) & \mathrm{H}(12) & 0.95(3) \\ \mathrm{C}(16) & \mathrm{H}(13) & 0.95(5) & \mathrm{C}(17) & \mathrm{H}(14) & 0.95(5) \\ \mathrm{C}(18) & \mathrm{H}(15) & 0.95(3) & \mathrm{C}(20) & \mathrm{H}(16) & 0.95(3) \\ \mathrm{C}(21) & \mathrm{H}(17) & 0.95(4) & \mathrm{C}(22) & \mathrm{H}(18) & 0.95(3) \\ \mathrm{C}(23) & \mathrm{H}(19) & 0.95(3) & \mathrm{C}(24) & \mathrm{H}(20) & 0.95(4) \\ \mathrm{C}(25) & \mathrm{H}(21) & 0.95(4) & \mathrm{C}(25) & \mathrm{H}(22) & 0.95(3) \\ \mathrm{C}(26) & \mathrm{H}(23) & 0.95(4) & \mathrm{C}(26) & \mathrm{H}(24) & 0.95(5) \\ \mathrm{C}(26) & \mathrm{H}(25) & 0.95(5) & \mathrm{C}(27) & \mathrm{H}(26) & 0.95(3) \\ \mathrm{C}(27) & \mathrm{H}(27) & 0.95(3) & \mathrm{C}(28) & \mathrm{H}(28) & 0.95(3) \\ \mathrm{C}(28) & \mathrm{H}(29) & 0.95(4) & \mathrm{C}(28) & \mathrm{H}(30) & 0.95(4) \\ \mathrm{C}(29) & \mathrm{H}(31) & 0.95(4) & \mathrm{C}(29) & \mathrm{H}(32) & 0.95(5) \\ \mathrm{C}(30) & \mathrm{H}(33) & 0.95(5) & \mathrm{C}(30) & \mathrm{H}(34) & 0.95(5)\end{array}$




\begin{tabular}{|c|c|c|c|c|c|}
\hline$C(30)$ & $\mathrm{H}(35)$ & $0.95(5)$ & $\mathrm{C}(31)$ & $\mathrm{H}(36)$ & $0.95(4)$ \\
\hline $\mathrm{C}(31)$ & $\mathrm{H}(37)$ & $0.95(3)$ & $C(32)$ & $\mathrm{H}(38)$ & $0.95(5)$ \\
\hline$C(32)$ & $\mathrm{H}(39)$ & $0.95(5)$ & $C(32)$ & $\mathrm{H}(40)$ & $0.95(4)$ \\
\hline $\mathrm{C}(33)$ & $\mathrm{H}(41)$ & $0.95(4)$ & $C(33)$ & $\mathrm{H}(42)$ & $0.95(4)$ \\
\hline $\mathrm{C}(34)$ & $\mathrm{H}(43)$ & $0.95(4)$ & $C(34)$ & $\mathrm{H}(44)$ & $0.95(4)$ \\
\hline$C(34)$ & $\mathrm{H}(45)$ & $0.95(4)$ & $C(35)$ & $\mathrm{H}(46)$ & $0.95(3)$ \\
\hline$C(35)$ & $\mathrm{H}(47)$ & $0.95(4)$ & $C(36)$ & $\mathrm{H}(48)$ & $0.95(4)$ \\
\hline$C(36)$ & $\mathrm{H}(49)$ & $0.95(4)$ & $C(36)$ & $\mathrm{H}(50)$ & $0.95(4)$ \\
\hline $\mathrm{C}(37)$ & $\mathrm{H}(51)$ & $0.95(3)$ & $C(37)$ & $\mathrm{H}(52)$ & $0.95(3)$ \\
\hline $\mathrm{C}(38)$ & $\mathrm{H}(53)$ & $0.95(3)$ & $\mathrm{C}(38)$ & $\mathrm{H}(54)$ & $0.95(5)$ \\
\hline $\mathrm{C}(38)$ & $\mathrm{H}(55)$ & $0.95(4)$ & $C(39)$ & $\mathrm{H}(56)$ & $0.95(4)$ \\
\hline C(39) & $\mathrm{H}(57)$ & $0.95(3)$ & $\mathrm{C}(40)$ & $\mathrm{H}(58)$ & $0.95(3)$ \\
\hline$C(40)$ & $\mathrm{H}(59)$ & $0.95(5)$ & $\mathrm{C}(40)$ & $\mathrm{H}(60)$ & $0.95(3)$ \\
\hline$C(41)$ & $\mathrm{H}(61)$ & $0.95(3)$ & $\mathrm{C}(41)$ & $\mathrm{H}(62)$ & $0.95(4)$ \\
\hline$C(42)$ & $\mathrm{H}(63)$ & $0.95(6)$ & $C(42)$ & $\mathrm{H}(64)$ & $0.95(5)$ \\
\hline$C(42)$ & $\mathrm{H}(65)$ & $0.95(5)$ & $\mathrm{C}(44)$ & $\mathrm{H}(66)$ & $0.95(4)$ \\
\hline$C(45)$ & $\mathrm{H}(67)$ & $0.95(6)$ & $C(46)$ & $\mathrm{H}(68)$ & $0.95(5)$ \\
\hline$C(47)$ & $\mathrm{H}(69)$ & $0.95(6)$ & $\mathrm{C}(48)$ & $\mathrm{H}(70)$ & $0.95(5)$ \\
\hline$C(50)$ & $\mathrm{H}(75)$ & $0.95(3)$ & $\mathrm{C}(51)$ & $\mathrm{H}(74)$ & $0.95(4)$ \\
\hline$C(52)$ & $\mathrm{H}(73)$ & $0.95(3)$ & $\mathrm{C}(53)$ & $\mathrm{H}(72)$ & $0.95(3)$ \\
\hline$C(54)$ & $\mathrm{H}(71)$ & $0.95(4)$ & $C(56)$ & $\mathrm{H}(76)$ & $0.95(6)$ \\
\hline$C(57)$ & $\mathrm{H}(77)$ & $0.95(6)$ & $\mathrm{C}(58)$ & $\mathrm{H}(78)$ & $0.95(5)$ \\
\hline$C(59)$ & $\mathrm{H}(79)$ & $0.95(6)$ & $C(60)$ & $\mathrm{H}(80)$ & $0.95(5)$ \\
\hline $\mathrm{C}(61)$ & $\mathrm{H}(86)$ & $0.95(4)$ & $\mathrm{C}(61)$ & $\mathrm{H}(87)$ & $0.95(5)$ \\
\hline$C(62)$ & $\mathrm{H}(88)$ & $0.95(5)$ & $\mathrm{C}(62)$ & $\mathrm{H}(89)$ & $0.95(4)$ \\
\hline$C(62)$ & $\mathrm{H}(90)$ & $0.95(5)$ & $\mathrm{C}(63)$ & $\mathrm{H}(91)$ & $0.95(4)$ \\
\hline $\mathrm{C}(63)$ & $\mathrm{H}(92)$ & $0.95(5)$ & $C(64)$ & $\mathrm{H}(93)$ & $0.95(6)$ \\
\hline C(64) & $\mathrm{H}(94)$ & $0.95(4)$ & $C(64)$ & $\mathrm{H}(95)$ & $0.95(6)$ \\
\hline$C(65)$ & $\mathrm{H}(96)$ & $0.95(5)$ & $C(65)$ & $\mathrm{H}(97)$ & $0.95(4)$ \\
\hline$C(66)$ & $\mathrm{H}(98)$ & $0.95(5)$ & $C(66)$ & $\mathrm{H}(99)$ & $0.95(5)$ \\
\hline$C(66)$ & $\mathrm{H}(100)$ & $0.95(3)$ & $\mathrm{C}(67)$ & $\mathrm{H}(101)$ & $0.95(5)$ \\
\hline$C(67)$ & $\mathrm{H}(102)$ & $0.95(5)$ & $\mathrm{C}(68)$ & $\mathrm{H}(103)$ & $0.95(7)$ \\
\hline $\mathrm{C}(68)$ & $\mathrm{H}(104)$ & $0.95(7)$ & $\mathrm{C}(68)$ & $\mathrm{H}(105)$ & $0.95(6)$ \\
\hline C(69) & $\mathrm{H}(111)$ & $0.95(5)$ & $\mathrm{C}(69)$ & $\mathrm{H}(112)$ & $0.95(5)$ \\
\hline$C(70)$ & $\mathrm{H}(113)$ & $0.95(6)$ & $\mathrm{C}(70)$ & $\mathrm{H}(114)$ & $0.95(7)$ \\
\hline $\mathrm{C}(70)$ & $\mathrm{H}(115)$ & $0.95(7)$ & $\mathrm{C}(71)$ & $\mathrm{H}(107)$ & $0.95(6)$ \\
\hline $\mathrm{C}(71)$ & $\mathrm{H}(126)$ & $0.95(7)$ & $C(72)$ & $\mathrm{H}(106)$ & $0.95(8)$ \\
\hline $\mathrm{C}(72)$ & $\mathrm{H}(108)$ & $0.95(11)$ & $C(72)$ & $\mathrm{H}(110)$ & $0.95(11)$ \\
\hline$C(73)$ & $\mathrm{H}(116)$ & $0.95(5)$ & $C(73)$ & $\mathrm{H}(117)$ & $0.95(3)$ \\
\hline $\mathrm{C}(74)$ & $\mathrm{H}(118)$ & $0.95(6)$ & $C(74)$ & $\mathrm{H}(119)$ & $0.95(5)$ \\
\hline $\mathrm{C}(74)$ & $\mathrm{H}(120)$ & $0.95(4)$ & $C(75)$ & $\mathrm{H}(121)$ & $0.95(4)$ \\
\hline$C(75)$ & $\mathrm{H}(122)$ & $0.95(4)$ & $C(76)$ & $\mathrm{H}(123)$ & $0.95(5)$ \\
\hline$C(76)$ & $\mathrm{H}(124)$ & $0.95(4)$ & $C(76)$ & $\mathrm{H}(125)$ & $0.95(5)$ \\
\hline $\mathrm{C}(77)$ & H(109) & $0.95(3)$ & $\mathrm{C}(77)$ & $\mathrm{H}(127)$ & $0.95(4)$ \\
\hline $\mathrm{C}(78)$ & $\mathrm{H}(128)$ & $0.95(4)$ & $\mathrm{C}(78)$ & $\mathrm{H}(129)$ & $0.95(5)$ \\
\hline
\end{tabular}




$\begin{array}{llllll}\mathrm{C}(78) & \mathrm{H}(130) & 0.95(3) & \mathrm{C}(80) & \mathrm{H}(81) & 0.95(5) \\ \mathrm{C}(81) & \mathrm{H}(82) & 0.95(5) & \mathrm{C}(82) & \mathrm{H}(83) & 0.95(3) \\ \mathrm{C}(83) & \mathrm{H}(84) & 0.95(5) & \mathrm{C}(84) & \mathrm{H}(85) & 0.95(4)\end{array}$

Table 5. Bond angles (O)

\begin{tabular}{|c|c|c|c|c|c|c|c|}
\hline atom & atom & atom & angle & atom & atom & atom & angle \\
\hline $\operatorname{Pd}(1)$ & $\operatorname{Pt}(1)$ & $\mathrm{P}(2)$ & $110.46(8)$ & $\operatorname{Pd}(1)$ & $\operatorname{Pt}(1)$ & $\mathrm{P}(3)$ & 133.07(9) \\
\hline $\mathrm{P}(2)$ & $\operatorname{Pt}(1)$ & $\mathrm{P}(3)$ & $113.76(12)$ & $\operatorname{Pd}(1)$ & $\operatorname{Pt}(1)$ & $\operatorname{Si}(1)$ & $52.30(8)$ \\
\hline $\mathrm{P}(2)$ & $\operatorname{Pt}(1)$ & $\operatorname{Si}(1)$ & $107.42(12)$ & $\mathrm{P}(3)$ & $\operatorname{Pt}(1)$ & $\operatorname{Si}(1)$ & $123.72(11)$ \\
\hline $\operatorname{Pd}(1)$ & $\operatorname{Pt}(1)$ & $\operatorname{Si}(2)$ & $54.97(8)$ & $\mathrm{P}(2)$ & $\operatorname{Pt}(1)$ & $\operatorname{Si}(2)$ & $102.18(12)$ \\
\hline $\mathrm{P}(3)$ & $\operatorname{Pt}(1)$ & $\mathrm{Si}(2)$ & $100.14(11)$ & $\operatorname{Si}(1)$ & $\operatorname{Pt}(1)$ & $\mathrm{Si}(2)$ & $106.94(12)$ \\
\hline $\operatorname{Pd}(2)$ & $\operatorname{Pt}(2)$ & $\mathrm{P}(5)$ & $131.58(10)$ & $\operatorname{Pd}(2)$ & $\operatorname{Pt}(2)$ & $P(6)$ & $109.70(9)$ \\
\hline $\mathrm{P}(5)$ & $\operatorname{Pt}(2)$ & $\mathrm{P}(6)$ & 114.9(1) & $\operatorname{Pd}(2)$ & $\operatorname{Pt}(2)$ & $\operatorname{Si}(3)$ & $55.94(9)$ \\
\hline $\mathrm{P}(5)$ & $\operatorname{Pt}(2)$ & $\mathrm{Si}(3)$ & $96.6(1)$ & $P(6)$ & $\operatorname{Pt}(2)$ & $\mathrm{Si}(3)$ & $101.50(13)$ \\
\hline $\operatorname{Pd}(2)$ & $\operatorname{Pt}(2)$ & $\operatorname{Si}(4)$ & $53.26(9)$ & $\mathrm{P}(5)$ & $\operatorname{Pt}(2)$ & $\operatorname{Si}(4)$ & $123.8(2)$ \\
\hline $\mathrm{P}(6)$ & $\operatorname{Pt}(2)$ & $\mathrm{Si}(4)$ & 108.11(13) & $\operatorname{Si}(3)$ & $\operatorname{Pt}(2)$ & $\mathrm{Si}(4)$ & $108.69(13)$ \\
\hline $\operatorname{Pt}(1)$ & $\operatorname{Pd}(1)$ & $\mathrm{P}(1)$ & $155.20(9)$ & $\operatorname{Pt}(1)$ & $\operatorname{Pd}(1)$ & $\mathrm{Si}(1)$ & $56.19(8)$ \\
\hline $\mathrm{P}(1)$ & $\operatorname{Pd}(1)$ & $\mathrm{Si}(1)$ & $99.10(12)$ & $\operatorname{Pt}(1)$ & $\operatorname{Pd}(1)$ & $\mathrm{Si}(2)$ & $54.06(8)$ \\
\hline $\mathrm{P}(1)$ & $\operatorname{Pd}(1)$ & $\mathrm{Si}(2)$ & $149.78(12)$ & $\operatorname{Si}(1)$ & $\operatorname{Pd}(1)$ & $\operatorname{Si}(2)$ & $109.91(12)$ \\
\hline $\operatorname{Pt}(2)$ & $\operatorname{Pd}(2)$ & $\mathrm{P}(4)$ & $164.37(10)$ & $\operatorname{Pt}(2)$ & $\operatorname{Pd}(2)$ & $\operatorname{Si}(3)$ & $53.75(9)$ \\
\hline $\mathrm{P}(4)$ & $\operatorname{Pd}(2)$ & $\operatorname{Si}(3)$ & $141.55(13)$ & $\operatorname{Pt}(2)$ & $\operatorname{Pd}(2)$ & $\mathrm{Si}(4)$ & $55.71(10)$ \\
\hline $\mathrm{P}(4)$ & $\operatorname{Pd}(2)$ & $\mathrm{Si}(4)$ & 108.7(1) & $\operatorname{Si}(3)$ & $\operatorname{Pd}(2)$ & $\mathrm{Si}(4)$ & 109.0(1) \\
\hline $\operatorname{Pd}(1)$ & $\mathrm{P}(1)$ & $\mathrm{C}(25)$ & $113.8(5)$ & $\operatorname{Pd}(1)$ & $\mathrm{P}(1)$ & $\mathrm{C}(27)$ & $119.8(4)$ \\
\hline$C(25)$ & $\mathrm{P}(1)$ & $\mathrm{C}(27)$ & $102.9(6)$ & $\operatorname{Pd}(1)$ & $\mathrm{P}(1)$ & $\mathrm{C}(29)$ & $112.6(4)$ \\
\hline$C(25)$ & $\mathrm{P}(1)$ & $\mathrm{C}(29)$ & $102.9(7)$ & $\mathrm{C}(27)$ & $\mathrm{P}(1)$ & $\mathrm{C}(29)$ & $102.7(7)$ \\
\hline $\operatorname{Pt}(1)$ & $\mathrm{P}(2)$ & $\mathrm{C}(31)$ & $117.5(5)$ & $\operatorname{Pt}(1)$ & $\mathrm{P}(2)$ & $C(33)$ & $118.9(5)$ \\
\hline $\mathrm{C}(31)$ & $\mathrm{P}(2)$ & $\mathrm{C}(33)$ & $98.6(7)$ & $\operatorname{Pt}(1)$ & $\mathrm{P}(2)$ & $C(35)$ & 115.1(4) \\
\hline $\mathrm{C}(31)$ & $\mathrm{P}(2)$ & $C(35)$ & $101.5(7)$ & $\mathrm{C}(33)$ & $\mathrm{P}(2)$ & $C(35)$ & $102.3(7)$ \\
\hline $\operatorname{Pt}(1)$ & $\mathrm{P}(3)$ & $\mathrm{C}(37)$ & $115.2(5)$ & $\operatorname{Pt}(1)$ & $\mathrm{P}(3)$ & $\mathrm{C}(39)$ & $121.4(4)$ \\
\hline $\mathrm{C}(37)$ & $\mathrm{P}(3)$ & $\mathrm{C}(39)$ & $99.9(6)$ & $\operatorname{Pt}(1)$ & $\mathrm{P}(3)$ & $\mathrm{C}(41)$ & $114.5(4)$ \\
\hline $\mathrm{C}(37)$ & $\mathrm{P}(3)$ & $\mathrm{C}(41)$ & $100.8(6)$ & $\mathrm{C}(39)$ & $\mathrm{P}(3)$ & $\mathrm{C}(41)$ & $102.0(7)$ \\
\hline $\operatorname{Pd}(2)$ & $\mathrm{P}(4)$ & $\mathrm{C}(61)$ & $117.2(6)$ & $\operatorname{Pd}(2)$ & $\mathrm{P}(4)$ & $\mathrm{C}(63)$ & $111.6(6)$ \\
\hline $\mathrm{C}(61)$ & $\mathrm{P}(4)$ & $\mathrm{C}(63)$ & $101.5(9)$ & $\operatorname{Pd}(2)$ & $\mathrm{P}(4)$ & $C(65)$ & $118.7(5)$ \\
\hline $\mathrm{C}(61)$ & $\mathrm{P}(4)$ & $C(65)$ & $102.6(8)$ & $\mathrm{C}(63)$ & $\mathrm{P}(4)$ & $C(65)$ & $102.9(8)$ \\
\hline $\operatorname{Pt}(2)$ & $\mathrm{P}(5)$ & $\mathrm{C}(67)$ & $120.5(6)$ & $\operatorname{Pt}(2)$ & $\mathrm{P}(5)$ & $\mathrm{C}(69)$ & $116.9(7)$ \\
\hline $\mathrm{C}(67)$ & $\mathrm{P}(5)$ & $\mathrm{C}(69)$ & $99.5(8)$ & $\operatorname{Pt}(2)$ & $\mathrm{P}(5)$ & $\mathrm{C}(71)$ & $115.1(7)$ \\
\hline$C(67)$ & $\mathrm{P}(5)$ & $\mathrm{C}(71)$ & $99.0(11)$ & $\mathrm{C}(69)$ & $\mathrm{P}(5)$ & $\mathrm{C}(71)$ & $102.5(13)$ \\
\hline $\operatorname{Pt}(2)$ & $\mathrm{P}(6)$ & $C(73)$ & $116.5(5)$ & $\operatorname{Pt}(2)$ & $\mathrm{P}(6)$ & $C(75)$ & $115.6(6)$ \\
\hline$C(73)$ & $\mathrm{P}(6)$ & $C(75)$ & $102.7(7)$ & $\operatorname{Pt}(2)$ & $\mathrm{P}(6)$ & $C(77)$ & $120.2(5)$ \\
\hline$C(73)$ & $P(6)$ & $C(77)$ & $99.1(8)$ & $\mathrm{C}(75)$ & $\mathrm{P}(6)$ & $C(77)$ & $99.6(7)$ \\
\hline $\operatorname{Pt}(1)$ & $\operatorname{Si}(1)$ & $\operatorname{Pd}(1)$ & $71.52(10)$ & $\operatorname{Pt}(1)$ & $\operatorname{Si}(1)$ & $\mathrm{C}(1)$ & $119.4(4)$ \\
\hline $\operatorname{Pd}(1)$ & $\mathrm{Si}(1)$ & $\mathrm{C}(1)$ & 119.1(5) & $\operatorname{Pt}(1)$ & $\operatorname{Si}(1)$ & $C(7)$ & $126.1(4)$ \\
\hline $\operatorname{Pd}(1)$ & $\operatorname{Si}(1)$ & $\mathrm{C}(7)$ & $112.5(4)$ & $\mathrm{C}(1)$ & $\operatorname{Si}(1)$ & $C(7)$ & $105.2(6)$ \\
\hline $\operatorname{Pt}(1)$ & $\operatorname{Si}(2)$ & $\operatorname{Pd}(1)$ & $70.97(10)$ & $\operatorname{Pt}(1)$ & $\operatorname{Si}(2)$ & $C(13)$ & $121.0(4)$ \\
\hline
\end{tabular}




\begin{tabular}{|c|c|c|c|c|c|c|c|}
\hline $\operatorname{Pd}(1)$ & $\operatorname{Si}(2)$ & $\mathrm{C}(13)$ & $124.5(4)$ & $\operatorname{Pt}(1)$ & $\operatorname{Si}(2)$ & $C(19)$ & $118.9(4)$ \\
\hline $\operatorname{Pd}(1)$ & $\operatorname{Si}(2)$ & $\mathrm{C}(19)$ & $118.0(4)$ & $\mathrm{C}(13)$ & $\operatorname{Si}(2)$ & $C(19)$ & $102.5(6)$ \\
\hline $\operatorname{Pt}(2)$ & $\operatorname{Si}(3)$ & $\operatorname{Pd}(2)$ & $70.31(10)$ & $\operatorname{Pt}(2)$ & $\operatorname{Si}(3)$ & $\mathrm{C}(43)$ & $119.3(4)$ \\
\hline $\operatorname{Pd}(2)$ & $\operatorname{Si}(3)$ & $C(43)$ & $127.0(5)$ & $\operatorname{Pt}(2)$ & $\operatorname{Si}(3)$ & $\mathrm{C}(49)$ & $120.0(4)$ \\
\hline $\operatorname{Pd}(2)$ & $\operatorname{Si}(3)$ & $\mathrm{C}(49)$ & $113.0(5)$ & $\mathrm{C}(43)$ & $\operatorname{Si}(3)$ & $C(49)$ & $105.1(6)$ \\
\hline $\operatorname{Pt}(2)$ & $\operatorname{Si}(4)$ & $\operatorname{Pd}(2)$ & $71.02(11)$ & $\operatorname{Pt}(2)$ & $\operatorname{Si}(4)$ & $C(55)$ & $123.0(5)$ \\
\hline $\operatorname{Pd}(2)$ & $\operatorname{Si}(4)$ & $C(55)$ & $118.2(5)$ & $\operatorname{Pt}(2)$ & $\operatorname{Si}(4)$ & $\mathrm{C}(79)$ & $121.6(5)$ \\
\hline $\operatorname{Pd}(2)$ & $\operatorname{Si}(4)$ & $\mathrm{C}(79)$ & $119.5(5)$ & $\mathrm{C}(55)$ & $\operatorname{Si}(4)$ & $\mathrm{C}(79)$ & $102.5(6)$ \\
\hline $\operatorname{Si}(1)$ & $\mathrm{C}(1)$ & $\mathrm{C}(2)$ & $122.2(11)$ & $\operatorname{Si}(1)$ & $\mathrm{C}(1)$ & $C(6)$ & $123.6(10)$ \\
\hline$C(2)$ & $\mathrm{C}(1)$ & $C(6)$ & $113.8(12)$ & $\mathrm{C}(1)$ & $\mathrm{C}(2)$ & $\mathrm{C}(3)$ & $123.4(15)$ \\
\hline$C(2)$ & $\mathrm{C}(3)$ & $\mathrm{C}(4)$ & $122.2(16)$ & $\mathrm{C}(3)$ & $\mathrm{C}(4)$ & $C(5)$ & $117.6(14)$ \\
\hline$C(4)$ & $C(5)$ & $C(6)$ & $117.8(14)$ & $\mathrm{C}(1)$ & $\mathrm{C}(6)$ & $\mathrm{C}(5)$ & $125.1(13)$ \\
\hline $\operatorname{Si}(1)$ & $\mathrm{C}(7)$ & $\mathrm{C}(8)$ & $122.3(9)$ & $\operatorname{Si}(1)$ & $\mathrm{C}(7)$ & $\mathrm{C}(12)$ & $120.9(9)$ \\
\hline $\mathrm{C}(8)$ & $C(7)$ & $\mathrm{C}(12)$ & 116.3(11) & $C(7)$ & $\mathrm{C}(8)$ & $\mathrm{C}(9)$ & $120.7(12)$ \\
\hline $\mathrm{C}(8)$ & $\mathrm{C}(9)$ & $\mathrm{C}(10)$ & $122.4(13)$ & $\mathrm{C}(9)$ & $C(10)$ & $\mathrm{C}(11)$ & $117.6(13)$ \\
\hline$C(10)$ & $\mathrm{C}(11)$ & $\mathrm{C}(12)$ & $119.8(13)$ & $C(7)$ & $\mathrm{C}(12)$ & $\mathrm{C}(11)$ & $123.1(12)$ \\
\hline $\operatorname{Si}(2)$ & $C(13)$ & $\mathrm{C}(14)$ & $120.8(10)$ & $\operatorname{Si}(2)$ & $\mathrm{C}(13)$ & $\mathrm{C}(18)$ & $120.5(10)$ \\
\hline$C(14)$ & $\mathrm{C}(13)$ & $\mathrm{C}(18)$ & $118.6(13)$ & $\mathrm{C}(13)$ & $\mathrm{C}(14)$ & $C(15)$ & $122.0(14)$ \\
\hline$C(14)$ & $C(15)$ & $\mathrm{C}(16)$ & $119.9(14)$ & $C(15)$ & $C(16)$ & $\mathrm{C}(17)$ & $117.8(14)$ \\
\hline$C(16)$ & $C(17)$ & $\mathrm{C}(18)$ & $121.1(15)$ & $\mathrm{C}(13)$ & $\mathrm{C}(18)$ & $\mathrm{C}(17)$ & $120.5(14)$ \\
\hline $\operatorname{Si}(2)$ & $C(19)$ & $\mathrm{C}(20)$ & $118.7(9)$ & $\operatorname{Si}(2)$ & $C(19)$ & $\mathrm{C}(24)$ & $124.3(10)$ \\
\hline$C(20)$ & $C(19)$ & $\mathrm{C}(24)$ & $116.9(12)$ & $C(19)$ & $C(20)$ & $\mathrm{C}(21)$ & $121.3(12)$ \\
\hline$C(20)$ & $\mathrm{C}(21)$ & $\mathrm{C}(22)$ & $118.8(14)$ & $\mathrm{C}(21)$ & $\mathrm{C}(22)$ & $\mathrm{C}(23)$ & $120.2(13)$ \\
\hline$C(22)$ & $\mathrm{C}(23)$ & $\mathrm{C}(24)$ & $119.9(13)$ & $\mathrm{C}(19)$ & $\mathrm{C}(24)$ & $\mathrm{C}(23)$ & $122.7(13)$ \\
\hline $\mathrm{P}(1)$ & $C(25)$ & $C(26)$ & $111.9(11)$ & $\mathrm{P}(1)$ & $\mathrm{C}(27)$ & $\mathrm{C}(28)$ & $118.6(10)$ \\
\hline $\mathrm{P}(1)$ & $C(29)$ & $\mathrm{C}(30)$ & $112.1(11)$ & $\mathrm{P}(2)$ & $\mathrm{C}(31)$ & $C(32)$ & $117.3(11)$ \\
\hline $\mathrm{P}(2)$ & $C(33)$ & $\mathrm{C}(34)$ & $115.2(10)$ & $\mathrm{P}(2)$ & $C(35)$ & $C(36)$ & $116.0(10)$ \\
\hline $\mathrm{P}(3)$ & $C(37)$ & $\mathrm{C}(38)$ & $114.5(9)$ & $\mathrm{P}(3)$ & C(39) & $\mathrm{C}(40)$ & $114.5(10)$ \\
\hline $\mathrm{P}(3)$ & $\mathrm{C}(41)$ & $\mathrm{C}(42)$ & $121.7(12)$ & $\operatorname{Si}(3)$ & $\mathrm{C}(43)$ & $\mathrm{C}(44)$ & $121.0(12)$ \\
\hline $\operatorname{Si}(3)$ & $\mathrm{C}(43)$ & $\mathrm{C}(48)$ & $121.1(13)$ & $\mathrm{C}(44)$ & $\mathrm{C}(43)$ & $\mathrm{C}(48)$ & $117.9(16)$ \\
\hline $\mathrm{C}(43)$ & $\mathrm{C}(44)$ & $\mathrm{C}(45)$ & $121.3(16)$ & $\mathrm{C}(44)$ & $C(45)$ & $C(46)$ & $119.4(18)$ \\
\hline$C(45)$ & $C(46)$ & $\mathrm{C}(47)$ & $120.2(18)$ & $C(46)$ & $\mathrm{C}(47)$ & $\mathrm{C}(48)$ & $119.7(18)$ \\
\hline $\mathrm{C}(43)$ & $\mathrm{C}(48)$ & $\mathrm{C}(47)$ & $121.4(18)$ & $\operatorname{Si}(3)$ & $\mathrm{C}(49)$ & $\mathrm{C}(50)$ & $119.2(11)$ \\
\hline $\operatorname{Si}(3)$ & $C(49)$ & $\mathrm{C}(54)$ & $124.3(11)$ & $\mathrm{C}(50)$ & $\mathrm{C}(49)$ & $\mathrm{C}(54)$ & $116.5(13)$ \\
\hline$C(49)$ & $\mathrm{C}(50)$ & $\mathrm{C}(51)$ & $122.2(15)$ & $\mathrm{C}(50)$ & $\mathrm{C}(51)$ & $C(52)$ & $120.1(16)$ \\
\hline$C(51)$ & $C(52)$ & $\mathrm{C}(53)$ & $120.4(15)$ & $C(52)$ & $\mathrm{C}(53)$ & $\mathrm{C}(54)$ & $118.3(15)$ \\
\hline $\mathrm{C}(49)$ & $C(54)$ & $\mathrm{C}(53)$ & $122.6(14)$ & $\operatorname{Si}(4)$ & $C(55)$ & $C(56)$ & $117.9(12)$ \\
\hline $\operatorname{Si}(4)$ & $C(55)$ & $\mathrm{C}(60)$ & $125.3(13)$ & $C(56)$ & $C(55)$ & $C(60)$ & $116.8(15)$ \\
\hline$C(55)$ & $C(56)$ & $C(57)$ & $120.8(18)$ & $C(56)$ & $C(57)$ & $\mathrm{C}(58)$ & $121.2(21)$ \\
\hline$C(57)$ & $\mathrm{C}(58)$ & $\mathrm{C}(59)$ & $118.8(19)$ & $\mathrm{C}(58)$ & $\mathrm{C}(59)$ & $C(60)$ & $120.5(19)$ \\
\hline$C(55)$ & $C(60)$ & $\mathrm{C}(59)$ & $122.1(17)$ & $\mathrm{P}(4)$ & $\mathrm{C}(61)$ & $C(62)$ & $112.3(12)$ \\
\hline $\mathrm{P}(4)$ & $C(63)$ & $C(64)$ & $112.7(11)$ & $\mathrm{P}(4)$ & $C(65)$ & $C(66)$ & $116.2(11)$ \\
\hline$P(5)$ & $C(67)$ & $\mathrm{C}(68)$ & $114.6(13)$ & $\mathrm{P}(5)$ & $\mathrm{C}(69)$ & $\mathrm{C}(70)$ & $121.2(20)$ \\
\hline $\mathrm{P}(5)$ & $\mathrm{C}(71)$ & $\mathrm{C}(72)$ & $116.0(24)$ & $\mathrm{P}(6)$ & $C(73)$ & $\mathrm{C}(74)$ & $119.3(12)$ \\
\hline$P(6)$ & $C(75)$ & $\mathrm{C}(76)$ & $115.7(11)$ & $\mathrm{P}(6)$ & $C(77)$ & $\mathrm{C}(78)$ & $113.9(10)$ \\
\hline
\end{tabular}




$\begin{array}{llllllll}\mathrm{Si}(4) & \mathrm{C}(79) & \mathrm{C}(80) & 120.4(11) & \mathrm{Si}(4) & \mathrm{C}(79) & \mathrm{C}(84) & 121.9(10) \\ \mathrm{C}(80) & \mathrm{C}(79) & \mathrm{C}(84) & 117.7(13) & \mathrm{C}(79) & \mathrm{C}(80) & \mathrm{C}(81) & 120.2(15) \\ \mathrm{C}(80) & \mathrm{C}(81) & \mathrm{C}(82) & 120.9(14) & \mathrm{C}(81) & \mathrm{C}(82) & \mathrm{C}(83) & 118.9(13) \\ \mathrm{C}(82) & \mathrm{C}(83) & \mathrm{C}(84) & 120.1(14) & \mathrm{C}(79) & \mathrm{C}(84) & \mathrm{C}(83) & 122.1(13)\end{array}$

Table 6. Bond angles involving hydrogens (O)

\begin{tabular}{|c|c|c|c|c|c|c|c|}
\hline atom & atom & atom & angle & atom & atom & atom & angle \\
\hline $\operatorname{Pd}(2)$ & $\operatorname{Pt}(2)$ & $\mathrm{H}(131)$ & $72.2(50)$ & $\mathrm{P}(5)$ & $\operatorname{Pt}(2)$ & $\mathrm{H}(131)$ & $101.5(50)$ \\
\hline $\mathrm{P}(6)$ & $\operatorname{Pt}(2)$ & $\mathrm{H}(131)$ & $117.9(50)$ & $\operatorname{Si}(3)$ & $\operatorname{Pt}(2)$ & $\mathrm{H}(131)$ & $123.0(50)$ \\
\hline $\operatorname{Si}(4)$ & $\operatorname{Pt}(2)$ & $\mathrm{H}(131)$ & $23.4(50)$ & $\operatorname{Pt}(2)$ & $\operatorname{Pd}(2)$ & $\mathrm{H}(132)$ & $78.4(63)$ \\
\hline $\mathrm{P}(4)$ & $\operatorname{Pd}(2)$ & $\mathrm{H}(132)$ & $117.3(63)$ & $\operatorname{Si}(3)$ & $\operatorname{Pd}(2)$ & $\mathrm{H}(132)$ & $26.4(63)$ \\
\hline $\operatorname{Si}(4)$ & $\operatorname{Pd}(2)$ & $\mathrm{H}(132)$ & $134.1(63)$ & $\operatorname{Pt}(2)$ & $\operatorname{Si}(3)$ & $\mathrm{H}(132)$ & $104.6(85)$ \\
\hline $\operatorname{Pd}(2)$ & $\operatorname{Si}(3)$ & $\mathrm{H}(132)$ & $37.1(85)$ & $\mathrm{C}(43)$ & $\operatorname{Si}(3)$ & $\mathrm{H}(132)$ & $97.7(85)$ \\
\hline$C(49)$ & $\operatorname{Si}(3)$ & $\mathrm{H}(132)$ & $107.2(85)$ & $\operatorname{Pt}(2)$ & $\mathrm{Si}(4)$ & $\mathrm{H}(131)$ & $22.7(49)$ \\
\hline $\operatorname{Pd}(2)$ & $\operatorname{Si}(4)$ & $\mathrm{H}(131)$ & $88.8(49)$ & $\mathrm{C}(55)$ & $\mathrm{Si}(4)$ & $\mathrm{H}(131)$ & $102.4(49)$ \\
\hline$C(79)$ & $\operatorname{Si}(4)$ & $\mathrm{H}(131)$ & $125.3(49)$ & $\mathrm{C}(1)$ & $\mathrm{C}(2)$ & $\mathrm{H}(1)$ & $118.2(18)$ \\
\hline $\mathrm{C}(3)$ & $\mathrm{C}(2)$ & $\mathrm{H}(1)$ & $118.4(19)$ & $\mathrm{C}(2)$ & $\mathrm{C}(3)$ & $\mathrm{H}(2)$ & $119.0(20)$ \\
\hline $\mathrm{C}(4)$ & $\mathrm{C}(3)$ & $\mathrm{H}(2)$ & $118.9(21)$ & $\mathrm{C}(3)$ & $\mathrm{C}(4)$ & $\mathrm{H}(3)$ & $121.0(22)$ \\
\hline$C(5)$ & $\mathrm{C}(4)$ & $\mathrm{H}(3)$ & $121.4(22)$ & $\mathrm{C}(4)$ & $C(5)$ & $\mathrm{H}(4)$ & $121.2(20)$ \\
\hline$C(6)$ & $\mathrm{C}(5)$ & $\mathrm{H}(4)$ & $121.0(20)$ & $\mathrm{C}(1)$ & $\mathrm{C}(6)$ & $\mathrm{H}(5)$ & $117.1(17)$ \\
\hline $\mathrm{C}(5)$ & $\mathrm{C}(6)$ & $\mathrm{H}(5)$ & $117.9(17)$ & $\mathrm{C}(7)$ & $\mathrm{C}(8)$ & $\mathrm{H}(6)$ & $119.4(16)$ \\
\hline $\mathrm{C}(9)$ & $\mathrm{C}(8)$ & $\mathrm{H}(6)$ & $119.9(17)$ & $\mathrm{C}(8)$ & $\mathrm{C}(9)$ & $\mathrm{H}(7)$ & $118.7(17)$ \\
\hline$C(10)$ & $\mathrm{C}(9)$ & $\mathrm{H}(7)$ & $118.9(18)$ & $\mathrm{C}(9)$ & $\mathrm{C}(10)$ & $\mathrm{H}(8)$ & $121.0(18)$ \\
\hline $\mathrm{C}(11)$ & $\mathrm{C}(10)$ & $\mathrm{H}(8)$ & $121.4(18)$ & $\mathrm{C}(10)$ & $\mathrm{C}(11)$ & $\mathrm{H}(9)$ & $120.0(19)$ \\
\hline$C(12)$ & $\mathrm{C}(11)$ & $\mathrm{H}(9)$ & $120.2(18)$ & $C(7)$ & $\mathrm{C}(12)$ & $\mathrm{H}(10)$ & $118.0(16)$ \\
\hline $\mathrm{C}(11)$ & $\mathrm{C}(12)$ & $\mathrm{H}(10)$ & $118.9(16)$ & $\mathrm{C}(13)$ & $\mathrm{C}(14)$ & $\mathrm{H}(11)$ & $118.9(18)$ \\
\hline$C(15)$ & $\mathrm{C}(14)$ & $\mathrm{H}(11)$ & 119.1(18) & $\mathrm{C}(14)$ & $\mathrm{C}(15)$ & $\mathrm{H}(12)$ & $120.2(21)$ \\
\hline$C(16)$ & $\mathrm{C}(15)$ & $\mathrm{H}(12)$ & $119.9(21)$ & $\mathrm{C}(15)$ & $C(16)$ & $\mathrm{H}(13)$ & $120.9(23)$ \\
\hline$C(17)$ & $\mathrm{C}(16)$ & $\mathrm{H}(13)$ & $121.3(23)$ & $\mathrm{C}(16)$ & $\mathrm{C}(17)$ & $\mathrm{H}(14)$ & $119.2(23)$ \\
\hline $\mathrm{C}(18)$ & $\mathrm{C}(17)$ & $\mathrm{H}(14)$ & $119.7(22)$ & $\mathrm{C}(13)$ & $\mathrm{C}(18)$ & $\mathrm{H}(15)$ & $119.4(19)$ \\
\hline$C(17)$ & $\mathrm{C}(18)$ & $\mathrm{H}(15)$ & $120.0(20)$ & $\mathrm{C}(19)$ & $\mathrm{C}(20)$ & $\mathrm{H}(16)$ & $119.0(16)$ \\
\hline $\mathrm{C}(21)$ & $\mathrm{C}(20)$ & $\mathrm{H}(16)$ & $119.6(17)$ & $\mathrm{C}(20)$ & $\mathrm{C}(21)$ & $\mathrm{H}(17)$ & $120.7(20)$ \\
\hline $\mathrm{C}(22)$ & $\mathrm{C}(21)$ & $\mathrm{H}(17)$ & $120.5(19)$ & $\mathrm{C}(21)$ & $\mathrm{C}(22)$ & $\mathrm{H}(18)$ & $119.9(16)$ \\
\hline $\mathrm{C}(23)$ & $\mathrm{C}(22)$ & $\mathrm{H}(18)$ & $119.8(16)$ & $\mathrm{C}(22)$ & $\mathrm{C}(23)$ & $\mathrm{H}(19)$ & $120.0(19)$ \\
\hline $\mathrm{C}(24)$ & $\mathrm{C}(23)$ & $\mathrm{H}(19)$ & 120.1(19) & $\mathrm{C}(19)$ & $\mathrm{C}(24)$ & $\mathrm{H}(20)$ & $118.4(16)$ \\
\hline$C(23)$ & $\mathrm{C}(24)$ & $\mathrm{H}(20)$ & $118.9(17)$ & $\mathrm{P}(1)$ & $\mathrm{C}(25)$ & $\mathrm{H}(21)$ & $109.2(15)$ \\
\hline$C(26)$ & $\mathrm{C}(25)$ & $\mathrm{H}(21)$ & $109.5(17)$ & $\mathrm{P}(1)$ & $\mathrm{C}(25)$ & $\mathrm{H}(22)$ & $108.7(15)$ \\
\hline$C(26)$ & $\mathrm{C}(25)$ & $\mathrm{H}(22)$ & 108.1(17) & $\mathrm{H}(21)$ & $\mathrm{C}(25)$ & $\mathrm{H}(22)$ & $109.5(20)$ \\
\hline$C(25)$ & $C(26)$ & $\mathrm{H}(23)$ & $108.4(20)$ & $\mathrm{C}(25)$ & $\mathrm{C}(26)$ & $\mathrm{H}(24)$ & $109.8(20)$ \\
\hline $\mathrm{H}(23)$ & $C(26)$ & $\mathrm{H}(24)$ & $109.5(25)$ & $\mathrm{C}(25)$ & $C(26)$ & $\mathrm{H}(25)$ & $110.3(20)$ \\
\hline $\mathrm{H}(23)$ & $C(26)$ & $\mathrm{H}(25)$ & $109.5(25)$ & $\mathrm{H}(24)$ & $C(26)$ & $\mathrm{H}(25)$ & $109.5(25)$ \\
\hline $\mathrm{P}(1)$ & $\mathrm{C}(27)$ & $\mathrm{H}(26)$ & $107.0(14)$ & $\mathrm{C}(28)$ & $\mathrm{C}(27)$ & $\mathrm{H}(26)$ & $107.2(15)$ \\
\hline $\mathrm{P}(1)$ & $\mathrm{C}(27)$ & $\mathrm{H}(27)$ & 107.1(14) & $\mathrm{C}(28)$ & $\mathrm{C}(27)$ & $\mathrm{H}(27)$ & $107.2(15)$ \\
\hline $\mathrm{H}(26)$ & $\mathrm{C}(27)$ & $\mathrm{H}(27)$ & $109.5(18)$ & $\mathrm{C}(27)$ & $\mathrm{C}(28)$ & $\mathrm{H}(28)$ & $109.5(17)$ \\
\hline
\end{tabular}




\begin{tabular}{|c|c|c|c|c|c|c|c|}
\hline$C(27)$ & $\mathrm{C}(28)$ & $\mathrm{H}(29)$ & $109.4(17)$ & $\mathrm{H}(28)$ & $\mathrm{C}(28)$ & $\mathrm{H}(29)$ & $109.5(21)$ \\
\hline $\mathrm{C}(27)$ & $\mathrm{C}(28)$ & $\mathrm{H}(30)$ & $109.4(17)$ & $\mathrm{H}(28)$ & $\mathrm{C}(28)$ & $\mathrm{H}(30)$ & $109.5(21)$ \\
\hline $\mathrm{H}(29)$ & $\mathrm{C}(28)$ & $\mathrm{H}(30)$ & $109.5(21)$ & $\mathrm{P}(1)$ & $\mathrm{C}(29)$ & $\mathrm{H}(31)$ & $108.7(16)$ \\
\hline$C(30)$ & $\mathrm{C}(29)$ & $\mathrm{H}(31)$ & $108.9(18)$ & $\mathrm{P}(1)$ & $\mathrm{C}(29)$ & $\mathrm{H}(32)$ & $108.9(16)$ \\
\hline$C(30)$ & $\mathrm{C}(29)$ & $\mathrm{H}(32)$ & $108.9(18)$ & $\mathrm{H}(31)$ & $\mathrm{C}(29)$ & $\mathrm{H}(32)$ & $109.5(21)$ \\
\hline $\mathrm{C}(29)$ & $\mathrm{C}(30)$ & $\mathrm{H}(33)$ & $109.6(21)$ & $C(29)$ & $\mathrm{C}(30)$ & $\mathrm{H}(34)$ & $109.4(21)$ \\
\hline $\mathrm{H}(33)$ & $\mathrm{C}(30)$ & $\mathrm{H}(34)$ & $109.5(25)$ & $\mathrm{C}(29)$ & $\mathrm{C}(30)$ & $\mathrm{H}(35)$ & $109.4(21)$ \\
\hline $\mathrm{H}(33)$ & $\mathrm{C}(30)$ & $\mathrm{H}(35)$ & $109.5(25)$ & $\mathrm{H}(34)$ & $\mathrm{C}(30)$ & $\mathrm{H}(35)$ & $109.5(25)$ \\
\hline $\mathrm{P}(2)$ & $\mathrm{C}(31)$ & $\mathrm{H}(36)$ & $107.4(16)$ & $\mathrm{C}(32)$ & $\mathrm{C}(31)$ & $\mathrm{H}(36)$ & $107.0(18)$ \\
\hline $\mathrm{P}(2)$ & $\mathrm{C}(31)$ & $\mathrm{H}(37)$ & $107.8(16)$ & $C(32)$ & $\mathrm{C}(31)$ & $\mathrm{H}(37)$ & $107.7(18)$ \\
\hline $\mathrm{H}(36)$ & $\mathrm{C}(31)$ & $\mathrm{H}(37)$ & $109.5(21)$ & $\mathrm{C}(31)$ & $\mathrm{C}(32)$ & $\mathrm{H}(38)$ & 108.6(19) \\
\hline $\mathrm{C}(31)$ & $C(32)$ & $\mathrm{H}(39)$ & $110.5(20)$ & $\mathrm{H}(38)$ & $\mathrm{C}(32)$ & $\mathrm{H}(39)$ & $109.5(24)$ \\
\hline $\mathrm{C}(31)$ & $\mathrm{C}(32)$ & $\mathrm{H}(40)$ & $109.3(20)$ & $\mathrm{H}(38)$ & $\mathrm{C}(32)$ & $\mathrm{H}(40)$ & $109.5(24)$ \\
\hline $\mathrm{H}(39)$ & $\mathrm{C}(32)$ & $\mathrm{H}(40)$ & $109.5(24)$ & $\mathrm{P}(2)$ & $\mathrm{C}(33)$ & $\mathrm{H}(41)$ & $108.0(16)$ \\
\hline $\mathrm{C}(34)$ & $\mathrm{C}(33)$ & $\mathrm{H}(41)$ & $107.8(17)$ & $\mathrm{P}(2)$ & $\mathrm{C}(33)$ & $\mathrm{H}(42)$ & $108.2(16)$ \\
\hline $\mathrm{C}(34)$ & $\mathrm{C}(33)$ & $\mathrm{H}(42)$ & 108.1(17) & $\mathrm{H}(41)$ & $\mathrm{C}(33)$ & $\mathrm{H}(42)$ & $109.5(21)$ \\
\hline $\mathrm{C}(33)$ & $\mathrm{C}(34)$ & $\mathrm{H}(43)$ & 109.1(17) & $\mathrm{C}(33)$ & $\mathrm{C}(34)$ & $\mathrm{H}(44)$ & $109.3(17)$ \\
\hline $\mathrm{H}(43)$ & $\mathrm{C}(34)$ & $\mathrm{H}(44)$ & $109.5(21)$ & $\mathrm{C}(33)$ & $\mathrm{C}(34)$ & $\mathrm{H}(45)$ & $110.0(17)$ \\
\hline $\mathrm{H}(43)$ & $\mathrm{C}(34)$ & $\mathrm{H}(45)$ & $109.5(21)$ & $\mathrm{H}(44)$ & $\mathrm{C}(34)$ & $\mathrm{H}(45)$ & $109.5(21)$ \\
\hline $\mathrm{P}(2)$ & $C(35)$ & $\mathrm{H}(46)$ & $107.7(14)$ & $C(36)$ & $C(35)$ & $\mathrm{H}(46)$ & $107.8(16)$ \\
\hline $\mathrm{P}(2)$ & $C(35)$ & $\mathrm{H}(47)$ & $107.7(14)$ & $\mathrm{C}(36)$ & $C(35)$ & $\mathrm{H}(47)$ & $108.1(16)$ \\
\hline $\mathrm{H}(46)$ & $C(35)$ & $\mathrm{H}(47)$ & 109.5(19) & $\mathrm{C}(35)$ & $C(36)$ & $\mathrm{H}(48)$ & 109.7(19) \\
\hline$C(35)$ & $\mathrm{C}(36)$ & $\mathrm{H}(49)$ & 109.2(19) & $\mathrm{H}(48)$ & $C(36)$ & $\mathrm{H}(49)$ & $109.5(23)$ \\
\hline$C(35)$ & $\mathrm{C}(36)$ & $\mathrm{H}(50)$ & $109.5(19)$ & $\mathrm{H}(48)$ & $C(36)$ & $\mathrm{H}(50)$ & $109.5(23)$ \\
\hline $\mathrm{H}(49)$ & $C(36)$ & $\mathrm{H}(50)$ & $109.5(23)$ & $\mathrm{P}(3)$ & $C(37)$ & $\mathrm{H}(51)$ & $108.3(14)$ \\
\hline $\mathrm{C}(38)$ & $\mathrm{C}(37)$ & $\mathrm{H}(51)$ & $107.9(15)$ & $\mathrm{P}(3)$ & $\mathrm{C}(37)$ & $\mathrm{H}(52)$ & $108.3(14)$ \\
\hline $\mathrm{C}(38)$ & $\mathrm{C}(37)$ & $\mathrm{H}(52)$ & $108.3(15)$ & $\mathrm{H}(51)$ & $C(37)$ & $\mathrm{H}(52)$ & $109.5(18)$ \\
\hline$C(37)$ & $\mathrm{C}(38)$ & $\mathrm{H}(53)$ & $109.3(17)$ & $\mathrm{C}(37)$ & $\mathrm{C}(38)$ & $\mathrm{H}(54)$ & $110.2(17)$ \\
\hline $\mathrm{H}(53)$ & $\mathrm{C}(38)$ & $\mathrm{H}(54)$ & $109.5(21)$ & $\mathrm{C}(37)$ & $\mathrm{C}(38)$ & $\mathrm{H}(55)$ & $108.9(17)$ \\
\hline $\mathrm{H}(53)$ & $\mathrm{C}(38)$ & $\mathrm{H}(55)$ & $109.5(21)$ & $\mathrm{H}(54)$ & $\mathrm{C}(38)$ & $\mathrm{H}(55)$ & $109.5(21)$ \\
\hline $\mathrm{P}(3)$ & $\mathrm{C}(39)$ & $\mathrm{H}(56)$ & $108.2(15)$ & $\mathrm{C}(40)$ & $C(39)$ & $\mathrm{H}(56)$ & $108.0(16)$ \\
\hline $\mathrm{P}(3)$ & $\mathrm{C}(39)$ & $\mathrm{H}(57)$ & $108.2(15)$ & $\mathrm{C}(40)$ & $C(39)$ & $\mathrm{H}(57)$ & $108.3(16)$ \\
\hline $\mathrm{H}(56)$ & $\mathrm{C}(39)$ & $\mathrm{H}(57)$ & $109.5(20)$ & $\mathrm{C}(39)$ & $\mathrm{C}(40)$ & $\mathrm{H}(58)$ & $110.0(18)$ \\
\hline C(39) & $\mathrm{C}(40)$ & $\mathrm{H}(59)$ & 109.1(18) & $\mathrm{H}(58)$ & $\mathrm{C}(40)$ & $\mathrm{H}(59)$ & $109.5(22)$ \\
\hline C(39) & $\mathrm{C}(40)$ & $\mathrm{H}(60)$ & $109.4(18)$ & $\mathrm{H}(58)$ & $\mathrm{C}(40)$ & $\mathrm{H}(60)$ & $109.5(22)$ \\
\hline $\mathrm{H}(59)$ & $\mathrm{C}(40)$ & $\mathrm{H}(60)$ & $109.5(22)$ & $\mathrm{P}(3)$ & $\mathrm{C}(41)$ & $\mathrm{H}(61)$ & $106.4(15)$ \\
\hline$C(42)$ & $\mathrm{C}(41)$ & $\mathrm{H}(61)$ & $106.2(18)$ & $\mathrm{P}(3)$ & $\mathrm{C}(41)$ & $\mathrm{H}(62)$ & $106.4(15)$ \\
\hline$C(42)$ & $\mathrm{C}(41)$ & $\mathrm{H}(62)$ & $106.3(18)$ & $\mathrm{H}(61)$ & $\mathrm{C}(41)$ & $\mathrm{H}(62)$ & $109.5(20)$ \\
\hline $\mathrm{C}(41)$ & $\mathrm{C}(42)$ & $\mathrm{H}(63)$ & $109.1(23)$ & $\mathrm{C}(41)$ & $\mathrm{C}(42)$ & $\mathrm{H}(64)$ & $109.0(23)$ \\
\hline $\mathrm{H}(63)$ & $\mathrm{C}(42)$ & $\mathrm{H}(64)$ & $109.5(29)$ & $\mathrm{C}(41)$ & $\mathrm{C}(42)$ & $\mathrm{H}(65)$ & $110.3(23)$ \\
\hline $\mathrm{H}(63)$ & $\mathrm{C}(42)$ & $\mathrm{H}(65)$ & $109.5(29)$ & $\mathrm{H}(64)$ & $\mathrm{C}(42)$ & $\mathrm{H}(65)$ & $109.5(29)$ \\
\hline$C(43)$ & $\mathrm{C}(44)$ & $\mathrm{H}(66)$ & $118.8(19)$ & $\mathrm{C}(45)$ & $\mathrm{C}(44)$ & $\mathrm{H}(66)$ & $119.9(20)$ \\
\hline $\mathrm{C}(44)$ & $\mathrm{C}(45)$ & $\mathrm{H}(67)$ & $120.3(22)$ & $\mathrm{C}(46)$ & $\mathrm{C}(45)$ & $\mathrm{H}(67)$ & $120.3(23)$ \\
\hline$C(45)$ & $C(46)$ & $\mathrm{H}(68)$ & $119.8(26)$ & $\mathrm{C}(47)$ & $\mathrm{C}(46)$ & $\mathrm{H}(68)$ & $119.9(27)$ \\
\hline$C(46)$ & $\mathrm{C}(47)$ & $\mathrm{H}(69)$ & $120.0(26)$ & $\mathrm{C}(48)$ & $\mathrm{C}(47)$ & $\mathrm{H}(69)$ & $120.3(25)$ \\
\hline
\end{tabular}




\begin{tabular}{|c|c|c|c|c|c|c|c|}
\hline$C(43)$ & $\mathrm{C}(48)$ & $\mathrm{H}(70)$ & $118.8(21)$ & $C(47)$ & $\mathrm{C}(48)$ & $\mathrm{H}(70)$ & $119.8(22)$ \\
\hline C(49) & $\mathrm{C}(50)$ & $\mathrm{H}(75)$ & $118.7(20)$ & $\mathrm{C}(51)$ & $C(50)$ & $\mathrm{H}(75)$ & $119.2(21)$ \\
\hline $\mathrm{C}(50)$ & $\mathrm{C}(51)$ & $\mathrm{H}(74)$ & $120.0(21)$ & $\mathrm{C}(52)$ & $C(51)$ & $\mathrm{H}(74)$ & $119.9(22)$ \\
\hline $\mathrm{C}(51)$ & $C(52)$ & $\mathrm{H}(73)$ & $120.0(22)$ & $\mathrm{C}(53)$ & $C(52)$ & $\mathrm{H}(73)$ & $119.6(21)$ \\
\hline$C(52)$ & $\mathrm{C}(53)$ & $\mathrm{H}(72)$ & $121.0(20)$ & $\mathrm{C}(54)$ & $\mathrm{C}(53)$ & $\mathrm{H}(72)$ & $120.7(20)$ \\
\hline C(49) & $\mathrm{C}(54)$ & $\mathrm{H}(71)$ & $118.4(19)$ & $\mathrm{C}(53)$ & $\mathrm{C}(54)$ & $\mathrm{H}(71)$ & $119.0(19)$ \\
\hline$C(55)$ & $\mathrm{C}(56)$ & $\mathrm{H}(76)$ & $119.0(23)$ & $\mathrm{C}(57)$ & $C(56)$ & $\mathrm{H}(76)$ & $120.2(25)$ \\
\hline$C(56)$ & $\mathrm{C}(57)$ & $\mathrm{H}(77)$ & $119.3(28)$ & $\mathrm{C}(58)$ & $\mathrm{C}(57)$ & $\mathrm{H}(77)$ & $119.6(28)$ \\
\hline$C(57)$ & $\mathrm{C}(58)$ & $\mathrm{H}(78)$ & $120.8(26)$ & $\mathrm{C}(59)$ & $\mathrm{C}(58)$ & $\mathrm{H}(78)$ & $120.5(26)$ \\
\hline $\mathrm{C}(58)$ & $\mathrm{C}(59)$ & $\mathrm{H}(79)$ & $119.9(25)$ & $\mathrm{C}(60)$ & $\mathrm{C}(59)$ & $\mathrm{H}(79)$ & $119.7(24)$ \\
\hline$C(55)$ & $\mathrm{C}(60)$ & $\mathrm{H}(80)$ & $118.3(22)$ & $\mathrm{C}(59)$ & $\mathrm{C}(60)$ & $\mathrm{H}(80)$ & $119.6(23)$ \\
\hline $\mathrm{P}(4)$ & $\mathrm{C}(61)$ & $\mathrm{H}(86)$ & $108.9(19)$ & $\mathrm{C}(62)$ & $\mathrm{C}(61)$ & $\mathrm{H}(86)$ & $108.7(21)$ \\
\hline $\mathrm{P}(4)$ & $\mathrm{C}(61)$ & $\mathrm{H}(87)$ & $108.6(19)$ & $\mathrm{C}(62)$ & $\mathrm{C}(61)$ & $\mathrm{H}(87)$ & $108.8(21)$ \\
\hline $\mathrm{H}(86)$ & $\mathrm{C}(61)$ & $\mathrm{H}(87)$ & $109.5(26)$ & $\mathrm{C}(61)$ & $\mathrm{C}(62)$ & $\mathrm{H}(88)$ & $109.4(22)$ \\
\hline $\mathrm{C}(61)$ & $\mathrm{C}(62)$ & $\mathrm{H}(89)$ & $109.4(22)$ & $\mathrm{H}(88)$ & $\mathrm{C}(62)$ & $\mathrm{H}(89)$ & $109.5(26)$ \\
\hline$C(61)$ & $\mathrm{C}(62)$ & $\mathrm{H}(90)$ & $109.6(22)$ & $\mathrm{H}(88)$ & $\mathrm{C}(62)$ & $\mathrm{H}(90)$ & $109.5(26)$ \\
\hline H(89) & $C(62)$ & $\mathrm{H}(90)$ & $109.5(26)$ & $\mathrm{P}(4)$ & $\mathrm{C}(63)$ & $\mathrm{H}(91)$ & 108.6(19) \\
\hline$C(64)$ & $\mathrm{C}(63)$ & $\mathrm{H}(91)$ & $108.8(21)$ & $\mathrm{P}(4)$ & $\mathrm{C}(63)$ & $\mathrm{H}(92)$ & $108.8(19)$ \\
\hline$C(64)$ & $\mathrm{C}(63)$ & $\mathrm{H}(92)$ & $108.4(21)$ & $\mathrm{H}(91)$ & $\mathrm{C}(63)$ & $\mathrm{H}(92)$ & $109.5(25)$ \\
\hline$C(63)$ & $\mathrm{C}(64)$ & $\mathrm{H}(93)$ & $110.2(23)$ & $\mathrm{C}(63)$ & $\mathrm{C}(64)$ & $\mathrm{H}(94)$ & $109.3(23)$ \\
\hline $\mathrm{H}(93)$ & $\mathrm{C}(64)$ & $\mathrm{H}(94)$ & $109.5(28)$ & $\mathrm{C}(63)$ & $C(64)$ & $\mathrm{H}(95)$ & $108.9(23)$ \\
\hline H(93) & $\mathrm{C}(64)$ & $\mathrm{H}(95)$ & $109.5(28)$ & $\mathrm{H}(94)$ & $\mathrm{C}(64)$ & $\mathrm{H}(95)$ & $109.5(28)$ \\
\hline $\mathrm{P}(4)$ & $\mathrm{C}(65)$ & $\mathrm{H}(96)$ & $107.8(16)$ & $\mathrm{C}(66)$ & $\mathrm{C}(65)$ & $\mathrm{H}(96)$ & $107.8(18)$ \\
\hline $\mathrm{P}(4)$ & $C(65)$ & $\mathrm{H}(97)$ & $107.8(16)$ & $\mathrm{C}(66)$ & $\mathrm{C}(65)$ & $\mathrm{H}(97)$ & $107.7(18)$ \\
\hline $\mathrm{H}(96)$ & $C(65)$ & $\mathrm{H}(97)$ & $109.5(21)$ & $C(65)$ & $\mathrm{C}(66)$ & $\mathrm{H}(98)$ & $109.0(20)$ \\
\hline$C(65)$ & $C(66)$ & $\mathrm{H}(99)$ & $109.1(20)$ & $\mathrm{H}(98)$ & $C(66)$ & $\mathrm{H}(99)$ & $109.5(24)$ \\
\hline$C(65)$ & $\mathrm{C}(66)$ & $\mathrm{H}(100)$ & $110.2(20)$ & $\mathrm{H}(98)$ & $\mathrm{C}(66)$ & $\mathrm{H}(100)$ & $109.5(24)$ \\
\hline H(99) & $C(66)$ & $\mathrm{H}(100)$ & $109.5(24)$ & $P(5)$ & $\mathrm{C}(67)$ & $\mathrm{H}(101)$ & $108.0(18)$ \\
\hline$C(68)$ & $\mathrm{C}(67)$ & $\mathrm{H}(101)$ & $108.4(21)$ & $P(5)$ & $\mathrm{C}(67)$ & $\mathrm{H}(102)$ & $108.3(18)$ \\
\hline$C(68)$ & $\mathrm{C}(67)$ & $\mathrm{H}(102)$ & $108.0(21)$ & $\mathrm{H}(101)$ & $\mathrm{C}(67)$ & $\mathrm{H}(102)$ & $109.5(24)$ \\
\hline$C(67)$ & $\mathrm{C}(68)$ & $\mathrm{H}(103)$ & $109.1(27)$ & $\mathrm{C}(67)$ & $\mathrm{C}(68)$ & $\mathrm{H}(104)$ & $109.5(27)$ \\
\hline H(103) & $\mathrm{C}(68)$ & $\mathrm{H}(104)$ & $109.5(34)$ & $\mathrm{C}(67)$ & $C(68)$ & $\mathrm{H}(105)$ & $109.7(27)$ \\
\hline $\mathrm{H}(103)$ & $\mathrm{C}(68)$ & $\mathrm{H}(105)$ & $109.5(34)$ & H(104) & $\mathrm{C}(68)$ & $\mathrm{H}(105)$ & $109.5(34)$ \\
\hline $\mathrm{P}(5)$ & $\mathrm{C}(69)$ & $\mathrm{H}(111)$ & $106.9(22)$ & $\mathrm{C}(70)$ & $\mathrm{C}(69)$ & $\mathrm{H}(111)$ & $106.3(26)$ \\
\hline$P(5)$ & $\mathrm{C}(69)$ & $\mathrm{H}(112)$ & $106.8(22)$ & $\mathrm{C}(70)$ & $\mathrm{C}(69)$ & $\mathrm{H}(112)$ & $105.9(26)$ \\
\hline $\mathrm{H}(111)$ & $\mathrm{C}(69)$ & $\mathrm{H}(112)$ & $109.5(29)$ & $\mathrm{C}(69)$ & $\mathrm{C}(70)$ & $\mathrm{H}(113)$ & $111.7(32)$ \\
\hline C(69) & $\mathrm{C}(70)$ & $\mathrm{H}(114)$ & $108.6(32)$ & $\mathrm{H}(113)$ & $\mathrm{C}(70)$ & $\mathrm{H}(114)$ & $109.5(39)$ \\
\hline C(69) & $\mathrm{C}(70)$ & $\mathrm{H}(115)$ & $108.1(32)$ & $\mathrm{H}(113)$ & $\mathrm{C}(70)$ & $\mathrm{H}(115)$ & $109.5(39)$ \\
\hline $\mathrm{H}(114)$ & $\mathrm{C}(70)$ & $\mathrm{H}(115)$ & $109.5(39)$ & $\mathrm{P}(5)$ & $\mathrm{C}(71)$ & $\mathrm{H}(107)$ & $108.2(28)$ \\
\hline$C(72)$ & $\mathrm{C}(71)$ & $\mathrm{H}(107)$ & $108.8(34)$ & $P(5)$ & $\mathrm{C}(71)$ & $\mathrm{H}(126)$ & $107.9(28)$ \\
\hline $\mathrm{C}(72)$ & $\mathrm{C}(71)$ & $\mathrm{H}(126)$ & $106.5(33)$ & $\mathrm{H}(107)$ & $\mathrm{C}(71)$ & $\mathrm{H}(126)$ & $109.5(38)$ \\
\hline $\mathrm{C}(71)$ & $\mathrm{C}(72)$ & $\mathrm{H}(106)$ & $109.9(44)$ & $\mathrm{C}(71)$ & $\mathrm{C}(72)$ & $\mathrm{H}(108)$ & $110.8(44)$ \\
\hline H(106) & $\mathrm{C}(72)$ & $\mathrm{H}(108)$ & $109.5(54)$ & $\mathrm{C}(71)$ & $\mathrm{C}(72)$ & $\mathrm{H}(110)$ & $107.6(44)$ \\
\hline $\mathrm{H}(106)$ & $\mathrm{C}(72)$ & $\mathrm{H}(110)$ & $109.5(54)$ & $\mathrm{H}(108)$ & $\mathrm{C}(72)$ & $\mathrm{H}(110)$ & $109.5(54)$ \\
\hline$P(6)$ & $\mathrm{C}(73)$ & $\mathrm{H}(116)$ & $107.0(17)$ & $\mathrm{C}(74)$ & $\mathrm{C}(73)$ & $\mathrm{H}(116)$ & $106.7(20)$ \\
\hline
\end{tabular}




$\begin{array}{llllllll}\mathrm{P}(6) & \mathrm{C}(73) & \mathrm{H}(117) & 107.1(18) & \mathrm{C}(74) & \mathrm{C}(73) & \mathrm{H}(117) & 107.1(20) \\ \mathrm{H}(116) & \mathrm{C}(73) & \mathrm{H}(117) & 109.4(24) & \mathrm{C}(73) & \mathrm{C}(74) & \mathrm{H}(118) & 108.8(22) \\ \mathrm{C}(73) & \mathrm{C}(74) & \mathrm{H}(119) & 110.4(23) & \mathrm{H}(118) & \mathrm{C}(74) & \mathrm{H}(119) & 109.5(28) \\ \mathrm{C}(73) & \mathrm{C}(74) & \mathrm{H}(120) & 109.2(22) & \mathrm{H}(118) & \mathrm{C}(74) & \mathrm{H}(120) & 109.5(28) \\ \mathrm{H}(119) & \mathrm{C}(74) & \mathrm{H}(120) & 109.5(28) & \mathrm{P}(6) & \mathrm{C}(75) & \mathrm{H}(121) & 108.2(16) \\ \mathrm{C}(76) & \mathrm{C}(75) & \mathrm{H}(121) & 107.7(18) & \mathrm{P}(6) & \mathrm{C}(75) & \mathrm{H}(122) & 107.9(16) \\ \mathrm{C}(76) & \mathrm{C}(75) & \mathrm{H}(122) & 107.7(18) & \mathrm{H}(121) & \mathrm{C}(75) & \mathrm{H}(122) & 109.5(22) \\ \mathrm{C}(75) & \mathrm{C}(76) & \mathrm{H}(123) & 109.1(21) & \mathrm{C}(75) & \mathrm{C}(76) & \mathrm{H}(124) & 110.3(21) \\ \mathrm{H}(123) & \mathrm{C}(76) & \mathrm{H}(124) & 109.5(25) & \mathrm{C}(75) & \mathrm{C}(76) & \mathrm{H}(125) & 109.1(21) \\ \mathrm{H}(123) & \mathrm{C}(76) & \mathrm{H}(125) & 109.5(25) & \mathrm{H}(124) & \mathrm{C}(76) & \mathrm{H}(125) & 109.5(25) \\ \mathrm{P}(6) & \mathrm{C}(77) & \mathrm{H}(109) & 108.4(16) & \mathrm{C}(78) & \mathrm{C}(77) & \mathrm{H}(109) & 108.1(17) \\ \mathrm{P}(6) & \mathrm{C}(77) & \mathrm{H}(127) & 108.6(16) & \mathrm{C}(78) & \mathrm{C}(77) & \mathrm{H}(127) & 108.4(17) \\ \mathrm{H}(109) & \mathrm{C}(77) & \mathrm{H}(127) & 109.5(21) & \mathrm{C}(77) & \mathrm{C}(78) & \mathrm{H}(128) & 109.2(17) \\ \mathrm{C}(77) & \mathrm{C}(78) & \mathrm{H}(129) & 110.3(17) & \mathrm{H}(128) & \mathrm{C}(78) & \mathrm{H}(129) & 109.5(21) \\ \mathrm{C}(77) & \mathrm{C}(78) & \mathrm{H}(130) & 108.9(17) & \mathrm{H}(128) & \mathrm{C}(78) & \mathrm{H}(130) & 109.5(21) \\ \mathrm{H}(129) & \mathrm{C}(78) & \mathrm{H}(130) & 109.5(21) & \mathrm{C}(79) & \mathrm{C}(80) & \mathrm{H}(81) & 119.8(21) \\ \mathrm{C}(81) & \mathrm{C}(80) & \mathrm{H}(81) & 120.0(21) & \mathrm{C}(80) & \mathrm{C}(81) & \mathrm{H}(82) & 119.5(22) \\ \mathrm{C}(82) & \mathrm{C}(81) & \mathrm{H}(82) & 119.6(22) & \mathrm{C}(81) & \mathrm{C}(82) & \mathrm{H}(83) & 120.6(19) \\ \mathrm{C}(83) & \mathrm{C}(82) & \mathrm{H}(83) & 120.6(19) & \mathrm{C}(82) & \mathrm{C}(83) & \mathrm{H}(84) & 120.1(19) \\ \mathrm{C}(84) & \mathrm{C}(83) & \mathrm{H}(84) & 119.8(19) & \mathrm{C}(79) & \mathrm{C}(84) & \mathrm{H}(85) & 118.7(17) \\ \mathrm{C}(83) & \mathrm{C}(84) & \mathrm{H}(85) & 119.2(17) & \mathrm{P}(2) & \mathrm{H}(131) & \mathrm{Si}(4) & 133.8(91) \\ \mathrm{Pd}(2) & \mathrm{H}(132) & \mathrm{Si}(3) & 116.5(13) & & & & \end{array}$




\section{Crystallographic Results of complex $\mathbf{3}$}

\section{Data Collection}

\section{Experimental}

A colorless prism crystal of $\mathrm{C}_{80} \mathrm{H}_{110} \mathrm{~N}_{4} \mathrm{P}_{2} \mathrm{Si}_{4} \mathrm{Pt}_{2}$ having approximate dimensions of $0.30 \times 0.15 \times 0.10 \mathrm{~mm}$ was mounted on a glass fiber. All measurements were made on a Rigaku Saturn CCD area detector with graphite monochromated Mo-K $\alpha$ radiation.

Indexing was performed from 720 images that were exposed for 10 seconds. The crystal-to-detector distance was $45.17 \mathrm{~mm}$.

Cell constants and an orientation matrix for data collection corresponded to a primitive triclinic cell with dimensions:

$$
\begin{array}{lll}
\mathrm{a}= & 11.787(3) \AA & \alpha=73.20(2)^{\mathrm{O}} \\
\mathrm{b}= & 12.124(3) \AA & \beta=81.52(2)^{\mathrm{O}} \\
\mathrm{c}= & 17.936(6) \AA & \gamma=58.98(1)^{\mathrm{O}} \\
\mathrm{V}=2102.8(10) \AA^{3} &
\end{array}
$$

For $\mathrm{Z}=1$ and F.W. $=1692.25$, the calculated density is $1.34 \mathrm{~g} / \mathrm{cm}^{3}$. Based on a statistical analysis of intensity distribution, and the successful solution and refinement of the structure, the space group was determined to be:

P1 (\#1)

The data were collected at a temperature of $20 \pm 1^{\circ} \mathrm{C}$ to a maximum $2 \theta$ value of $55.0^{\circ}$. A total of 720 oscillation images were collected. A sweep of data was done using $\omega$ scans from -110.0 to $70.0^{\circ}$ in $0.5^{\circ}$ step, at $\chi=45.0^{\circ}$ and $\phi=0.0^{\circ}$. The exposure rate was 10.0 [sec./O ]. The detector swing angle was $-20.26^{\circ}$. A second sweep was performed using $\omega$ scans from -110.0 to $70.0^{\circ}$ in $0.5^{\circ}$ step, at $\chi=45.0^{\circ}$ and $\phi=90.0^{\circ}$. The exposure rate was $10.0[\mathrm{sec} . / \mathrm{O}$ ]. The detector swing angle was $-20.26^{\circ}$. The crystal-to-detector distance was $45.17 \mathrm{~mm}$. Readout was performed in the $0.070 \mathrm{~mm}$ pixel mode.

\section{Data Reduction}

Of the 14255 reflections that were collected, 8664 were unique $(\mathrm{R} i n t=0.043)$; equivalent reflections were merged. Data were collected and processed using CrystalClear (Rigaku). Net intensities and sigmas were derived as follows:

$$
\begin{aligned}
& \mathrm{F}^{2}=\left[\Sigma\left(\mathrm{P}_{\mathrm{i}}-\mathrm{mB}_{\text {ave }}\right)\right] \cdot \mathrm{Lp}^{-1} \\
& \text { where } \mathrm{P}_{\mathrm{i}} \text { is the value in counts of the } \mathrm{i}^{\text {th }} \text { pixel } \\
& \mathrm{m} \text { is the number of pixels in the integration area } \\
& \mathrm{B}_{\text {ave }} \text { is the background average } \\
& \mathrm{Lp} \text { is the Lorentz and polarization factor } \\
& \mathrm{B}_{\text {ave }}=\Sigma\left(\mathrm{B}_{\mathrm{j}}\right) / \mathrm{n} \\
& \text { where } \mathrm{n} \text { is the number of pixels in the background area } \\
& \quad \mathrm{Bj}_{\mathrm{j}} \text { is the value of the } \mathrm{j}^{\text {th }} \text { pixel in counts } \\
& .2\left(\mathrm{~F}^{2} \mathrm{hkl}\right)=\left[\left(\Sigma \mathrm{P}_{\mathrm{i}}\right)+\mathrm{m}\left(\left(\Sigma\left(\mathrm{B}_{\text {ave }}-\mathrm{B}_{\mathrm{j}}\right)^{2}\right) /(\mathrm{n}-1)\right)\right] \cdot \mathrm{Lp} \cdot \text { errmul }+\left(\text { erradd } \cdot \mathrm{F}^{2}\right)^{2} \\
& \text { where erradd }=0.00 \\
& \text { errmul }=1.00
\end{aligned}
$$

The linear absorption coefficient, $\mu$, for Mo-K $\alpha$ radiation is $34.5 \mathrm{~cm}^{-1}$. An empirical 
absorption correction was applied which resulted in transmission factors ranging from 0.68 to 1.00. The data were corrected for Lorentz and polarization effects.

Structure Solution and Refinement

The structure was solved by direct methods 2 and expanded using Fourier techniques ${ }^{3}$. Some non-hydrogen atoms were refined anisotropically, while the rest were refined isotropically. Hydrogen atoms were refined using the riding model. The final cycle of full-matrix least-squares refinement ${ }^{4}$ on $\mathrm{F}$ was based on 6656 observed reflections (I $>3.00 \bullet(\mathrm{I})$ ) and 859 variable parameters and converged (largest parameter shift was 0.42 times its esd) with unweighted and weighted agreement factors of:

$$
\begin{gathered}
\mathrm{R}=\Sigma\|\mathrm{Fo}|-| \mathrm{Fc}\| / \Sigma|\mathrm{Fo}|=0.043 \\
\mathrm{R}_{\mathrm{W}}=\left[\Sigma \mathrm{w}(|\mathrm{Fo}|-|\mathrm{Fc}|)^{2} / \Sigma \mathrm{F} \mathrm{Fo}^{2}\right]^{1 / 2}=0.053
\end{gathered}
$$

The standard deviation of an observation of unit weight ${ }^{5}$ was 0.99 . A Sheldrick weighting scheme was used. Plots of $\Sigma \mathrm{w}(|\mathrm{Fo}|-|\mathrm{Fc}|)^{2}$ versus $|\mathrm{Fo}|$, reflection order in data collection, $\sin \theta / \lambda$ and various classes of indices showed no unusual trends. The maximum and minimum peaks on the final difference Fourier map corresponded to 2.64 and $-1.09 \mathrm{e} / \AA^{3}$, respectively.

Neutral atom scattering factors were taken from Cromer and Waber 6 . Anomalous dispersion effects were included in Fcalc 7 ; the values for $\Delta \mathrm{f}^{\prime}$ and $\Delta \mathrm{f}^{\prime \prime}$ were those of Creagh and McAuley ${ }^{8}$. The values for the mass attenuation coefficients are those of Creagh and Hubbell 9 . All calculations were performed using the CrystalStructure 10,11 crystallographic software package.

\section{References}

(1) CrystalClear: Rigaku Corporation, 1999. CrystalClear Software User's Guide, Molecular Structure Corporation, (c) 2000.J.W.Pflugrath (1999) Acta Cryst. D55, 1718-1725.

(2) SIR97: Altomare, A., Burla, M., Camalli, M., Cascarano, G., Giacovazzo, C., Guagliardi, A., Moliterni, A., Polidori, G., and Spagna, R. (1999). J. Appl. Cryst., 32, 115-119.

(3) DIRDIF99: Beurskens, P.T., Admiraal, G., Beurskens, G., Bosman, W.P., de Gelder, R., Israel, R. and Smits, J.M.M.(1999). The DIRDIF-99 program system, Technical Report of the Crystallography Laboratory, University of Nijmegen, The Netherlands.

(4) Least Squares function minimized:

$$
\Sigma w\left(\left|\mathrm{~F}_{\mathrm{O}}\right|-\left|\mathrm{F}_{\mathrm{C}}\right|\right)^{2} \quad \text { where } \mathrm{w}=\text { Least Squares weights. }
$$

(5) Standard deviation of an observation of unit weight:

$$
\begin{array}{ll}
{\left[\sum w\left(\left|\mathrm{~F}_{\mathrm{O}}\right|-\left|\mathrm{F}_{\mathrm{C}}\right|\right)^{2} /\left(\mathrm{N}_{\mathrm{O}}-\mathrm{N}_{\mathrm{V}}\right)\right]^{1 / 2}} \\
\text { where: } & \mathrm{N}_{\mathrm{O}}=\text { number of observations } \\
& \mathrm{N}_{\mathrm{V}}=\text { number of variables }
\end{array}
$$

(6) Cromer, D. T. \& Waber, J. T.; "International Tables for X-ray Crystallography", Vol. IV, The Kynoch Press, Birmingham, England, Table 2.2 A (1974).

(7) Ibers, J. A. \& Hamilton, W. C.; Acta Crystallogr., 17, 781 (1964).

(8) Creagh, D. C. \& McAuley, W.J .; "International Tables for Crystallography", Vol C, (A.J.C.

Wilson, ed.), Kluwer Academic Publishers, Boston, Table 4.2.6.8, pages 219-222 (1992).

(9) Creagh, D. C. \& Hubbell, J.H..; "International Tables for Crystallography", Vol C, (A.J.C. Wilson, ed.), Kluwer Academic Publishers, Boston, Table 4.2.4.3, pages 200-206 (1992). (10) CrystalStructure 3.5.1: Crystal Structure Analysis Package, Rigaku and Rigaku/MSC (2000-2003). 9009 New Trails Dr. The Woodlands TX 77381 USA.

(11) CRYSTALS Issue 10: Watkin, D.J., Prout, C.K. Carruthers, J.R. \& Betteridge, P.W. Chemical Crystallography Laboratory, Oxford, UK. (1996) 
EXPERIMENTAL DETAILS

Empirical Formula

Formula Weight

Crystal Color, Habit

A. Crystal Data

Crystal Dimensions

Crystal System

Lattice Type

Detector Position

Pixel Size

Lattice Parameters

Space Group

$Z$ value

$\mathrm{D}_{\text {calc }}$

F000

$\mu(\operatorname{MoK} \alpha)$

$\mathrm{C}_{80} \mathrm{H}_{110} \mathrm{~N}_{4} \mathrm{P}_{2} \mathrm{Si}_{4} \mathrm{Pt}_{2}$

1692.25

colorless, prism

0.30 X 0.15 X $0.10 \mathrm{~mm}$

triclinic

Primitive

$45.17 \mathrm{~mm}$

$0.035 \mathrm{~mm}$

$\mathrm{a}=11.787(3) \AA$

$\mathrm{b}=12.124(3) \AA$

$\mathrm{c}=17.936(6) \AA$

$\alpha=73.20(2)^{\mathrm{O}}$

$\beta=81.52(2)^{\mathrm{O}}$

$\gamma=58.98(1)^{\mathrm{O}}$

$\mathrm{V}=2102.8(10) \AA^{3}$

P1 (No.1)

1

$1.336 \mathrm{~g} / \mathrm{cm}^{3}$

860.00

$34.45 \mathrm{~cm}^{-1}$

B. Intensity Measurements

Detector

Goniometer

Radiation

Detector Aperture

Data Images

$\omega$ oscillation Range $(\chi=45.0, \phi=0.0)$

Exposure Rate

Detector Swing Angle

$\omega$ oscillation Range $(\chi=45.0,+=90.0)$

Exposure Rate

Detector Swing Angle

Detector Position

Pixel Size

$2 \theta_{\max }$

No. of Reflections Measured

Corrections
Rigaku Saturn

Rigaku AFC10

$\operatorname{MoK} \bullet(\bullet=0.71070 \AA)$

graphite monochromated

$70 \mathrm{~mm} \times 70 \mathrm{~mm}$

720 exposures

$-110.0-70.0^{\circ}$

$10.0 \mathrm{sec} . /^{\circ}$

$-20.26^{\circ}$

$-110.0-70.0^{\circ}$

$10.0 \mathrm{sec} . /^{\circ}$

$-20.26^{\circ}$

$45.17 \mathrm{~mm}$

$0.035 \mathrm{~mm}$

$55.0^{\circ}$

Total: 14255

Unique: $8664\left(\mathrm{R}_{\text {int }}=0.043\right)$

Lorentz-polarization

Absorption 
(trans. factors: $0.6774-1.0000$ )

Structure Solution

Refinement

Function Minimized

Least Squares Weights

Anomalous Dispersion

No. Observations $(\mathrm{I}>3.00 \bullet(\mathrm{I}))$

No. Variables

Reflection/Parameter Ratio

Residuals: R (I>3.00 $\sigma(\mathrm{I}))$

Residuals: Rw (I>3.00б(I))

Goodness of Fit Indicator

Max Shift/Error in Final Cycle

Maximum peak in Final Diff. Map

Minimum peak in Final Diff. Map
C. Structure Solution and Refinement

Direct Methods (SIR97)

Full-matrix least-squares on $\mathrm{F}$

$\Sigma \mathrm{w}(|\mathrm{Fo}|-|\mathrm{Fc}|)^{2}$

$1 /\left[0.0012 \mathrm{Fo}^{2}+1.0000 \bullet\left(\mathrm{Fo}^{2}\right)\right]$

All non-hydrogen atoms

6656

859

7.75

0.043

0.053

0.989

0.425

$2.64 \mathrm{e} / \AA^{3}$

$-1.09 \mathrm{e} / \AA^{3}$

Table 1. Atomic coordinates and $\mathrm{B}_{\mathrm{iso}} / \mathrm{B}_{\mathrm{eq}}$

$\begin{array}{lllll}\text { atom } & \mathrm{x} & \mathrm{y} & \mathrm{z} & \mathrm{B} \text { eq } \\ \mathrm{Pt}(1) & 0.5464(3) & 0.1848(3) & -0.5025(2) & 3.86(2) \\ \mathrm{Pt}(2) & 0.6176(3) & 0.6491(3) & -0.0022(2) & 3.80(2) \\ \mathrm{P}(1) & 0.7563(6) & 0.1438(7) & -0.4692(4) & 7.53(13) \\ \mathrm{P}(2) & 0.4113(6) & 0.8171(6) & 0.0304(4) & 7.73(13) \\ \mathrm{Si}(1) & 0.3394(4) & 0.2346(4) & -0.5382(3) & 3.94(7) \\ \mathrm{Si}(2) & 0.4589(4) & 0.4133(4) & -0.5627(3) & 4.07(7) \\ \mathrm{Si}(3) & 0.8297(4) & 0.4894(4) & -0.0383(3) & 4.01(7) \\ \mathrm{Si}(4) & 0.7085(4) & 0.7880(4) & -0.0623(3) & 4.02(7) \\ \mathrm{N}(1) & 0.2438(12) & 0.3997(13) & -0.5896(8) & 4.9(3) \\ \mathrm{N}(2) & 0.621(2) & -0.1151(11) & -0.4382(8) & 5.8(3) \\ \mathrm{N}(3) & 0.9244(13) & 0.558(2) & -0.0903(9) & 5.3(3) \\ \mathrm{N}(4) & 0.547(1) & 0.422(1) & 0.0614(8) & 5.5(3) \\ \mathrm{C}(1) & 0.323(1) & 0.462(1) & -0.6283(9) & 5.0(3) \\ \mathrm{C}(2) & 0.114(2) & 0.458(2) & -0.6280(12) & 5.6(4) \\ \mathrm{C}(3) & 0.126(2) & 0.467(3) & -0.716(2) & 10.7(8) \\ \mathrm{C}(4) & 0.028(2) & 0.608(2) & -0.624(2) & 9.9(6) \\ \mathrm{C}(5) & 0.037(2) & 0.392(3) & -0.590(2) & 9.9(7) \\ \mathrm{C}(6) & 0.595(2) & -0.004(1) & -0.4610(9) & 5.0(3) \\ \mathrm{C}(7) & 0.669(2) & -0.257(2) & -0.4214(12) & 6.5(4) \\ \mathrm{C}(8) & 0.588(7) & -0.271(4) & -0.376(4) & 26.1(23) \\ \mathrm{C}(9) & 0.659(7) & -0.287(3) & -0.491(2) & 22.8(22) \\ \mathrm{C}(10) & 0.798(6) & -0.330(3) & -0.398(6) & 33.1(34) \\ \mathrm{C}(11) & 0.248(1) & 0.197(2) & -0.4465(9) & 5.0(3)\end{array}$




\begin{tabular}{|c|c|c|c|c|}
\hline$C(12)$ & $0.172(2)$ & $0.292(2)$ & $-0.4025(11)$ & $6.5(5)$ \\
\hline $\mathrm{C}(13)$ & $0.105(2)$ & $0.270(3)$ & $-0.335(2)$ & $8.9(7)$ \\
\hline $\mathrm{C}(14)$ & $0.127(4)$ & $0.138(5)$ & $-0.308(2)$ & $11.9(11)$ \\
\hline$C(15)$ & $0.200(2)$ & $0.041(2)$ & $-0.3439(12)$ & $7.7(5)$ \\
\hline$C(16)$ & $0.259(2)$ & $0.073(2)$ & $-0.4142(10)$ & $6.0(4)$ \\
\hline $\mathrm{C}(17)$ & $0.372(3)$ & $0.115(2)$ & $-0.5970(12)$ & $6.0(5)$ \\
\hline $\mathrm{C}(18)$ & $0.287(2)$ & $0.065(2)$ & $-0.603(2)$ & $7.0(5)$ \\
\hline C(19) & $0.332(5)$ & $-0.025(2)$ & $-0.652(2)$ & $10.9(11)$ \\
\hline $\mathrm{C}(20)$ & $0.440(4)$ & $-0.066(3)$ & $-0.689(2)$ & $10.7(9)$ \\
\hline $\mathrm{C}(21)$ & $0.512(3)$ & $-0.011(2)$ & $-0.691(1)$ & $9.0(6)$ \\
\hline $\mathrm{C}(22)$ & $0.487(2)$ & $0.072(2)$ & $-0.6440(10)$ & $6.3(4)$ \\
\hline $\mathrm{C}(23)$ & $0.5731(13)$ & $0.4628(13)$ & $-0.6295(8)$ & $4.3(3)$ \\
\hline $\mathrm{C}(24)$ & $0.606(1)$ & $0.557(1)$ & $-0.6272(10)$ & $5.0(3)$ \\
\hline $\mathrm{C}(25)$ & $0.687(2)$ & $0.584(2)$ & $-0.6794(12)$ & $6.6(4)$ \\
\hline $\mathrm{C}(26)$ & $0.744(2)$ & $0.522(2)$ & $-0.7389(10)$ & $6.8(4)$ \\
\hline $\mathrm{C}(27)$ & $0.717(2)$ & $0.430(2)$ & $-0.7453(11)$ & $6.9(4)$ \\
\hline $\mathrm{C}(28)$ & $0.633(2)$ & $0.399(2)$ & $-0.6933(10)$ & $5.8(4)$ \\
\hline C(29) & $0.3791(12)$ & $0.5265(12)$ & $-0.4925(8)$ & $4.3(3)$ \\
\hline $\mathrm{C}(30)$ & $0.387(3)$ & $0.465(2)$ & $-0.4103(13)$ & $6.7(5)$ \\
\hline $\mathrm{C}(31)$ & $0.332(3)$ & $0.544(2)$ & $-0.358(2)$ & $8.1(6)$ \\
\hline $\mathrm{C}(32)$ & $0.262(4)$ & $0.682(4)$ & $-0.386(2)$ & $11.9(12)$ \\
\hline $\mathrm{C}(33)$ & $0.250(2)$ & $0.743(2)$ & $-0.466(2)$ & $9.4(7)$ \\
\hline $\mathrm{C}(34)$ & $0.309(2)$ & $0.660(2)$ & $-0.5150(11)$ & $6.2(4)$ \\
\hline $\mathrm{C}(35)$ & $0.858(2)$ & $0.002(2)$ & $-0.4006(13)$ & $7.3(4)$ \\
\hline $\mathrm{C}(36)$ & $0.791(4)$ & $0.003(4)$ & $-0.328(2)$ & $12.6(9)$ \\
\hline $\mathrm{C}(37)$ & $0.885(6)$ & $0.048(6)$ & $-0.525(4)$ & $20.8(19)$ \\
\hline $\mathrm{C}(38)$ & $0.894(5)$ & $-0.037(5)$ & $-0.569(3)$ & $17.0(14)$ \\
\hline C(39) & $0.828(6)$ & $0.227(6)$ & $-0.481(4)$ & $18.0(17)$ \\
\hline$C(40)$ & $0.753(4)$ & $0.314(4)$ & $-0.422(3)$ & $13.8(11)$ \\
\hline $\mathrm{C}(41)$ & $0.847(1)$ & $0.703(1)$ & $-0.1296(9)$ & $4.8(3)$ \\
\hline $\mathrm{C}(42)$ & $1.057(1)$ & $0.487(2)$ & $-0.1292(11)$ & $5.5(4)$ \\
\hline $\mathrm{C}(43)$ & $1.130(2)$ & $0.345(2)$ & $-0.087(2)$ & $10.0(6)$ \\
\hline $\mathrm{C}(44)$ & $1.043(3)$ & $0.510(3)$ & $-0.2155(13)$ & $9.8(7)$ \\
\hline$C(45)$ & $1.142(2)$ & $0.550(3)$ & $-0.122(2)$ & $9.2(7)$ \\
\hline $\mathrm{C}(46)$ & $0.575(2)$ & $0.508(2)$ & $0.0389(10)$ & $5.7(4)$ \\
\hline $\mathrm{C}(47)$ & $0.503(2)$ & $0.325(2)$ & $0.0774(12)$ & $6.1(3)$ \\
\hline $\mathrm{C}(48)$ & $0.491(6)$ & $0.300(6)$ & $0.006(4)$ & $18.9(18)$ \\
\hline C(49) & $0.597(7)$ & $0.220(7)$ & $0.119(5)$ & $21.4(23)$ \\
\hline $\mathrm{C}(50)$ & $0.370(5)$ & $0.380(6)$ & $0.111(3)$ & $17.4(16)$ \\
\hline $\mathrm{C}(51)$ & $0.921(1)$ & $0.361(1)$ & $0.0537(9)$ & $4.9(3)$ \\
\hline $\mathrm{C}(52)$ & $0.993(2)$ & $0.383(2)$ & $0.0956(12)$ & $6.5(5)$ \\
\hline $\mathrm{C}(53)$ & $1.058(2)$ & $0.291(4)$ & $0.166(2)$ & $10.3(8)$ \\
\hline $\mathrm{C}(54)$ & $1.044(4)$ & $0.174(3)$ & $0.192(2)$ & $10.9(8)$ \\
\hline $\mathrm{C}(55)$ & $0.970(2)$ & $0.152(2)$ & $0.1545(12)$ & $8.0(5)$ \\
\hline$C(56)$ & $0.908(2)$ & $0.247(1)$ & $0.0865(9)$ & $5.6(3)$ \\
\hline
\end{tabular}




\begin{tabular}{|c|c|c|c|c|}
\hline$C(57)$ & $0.796(2)$ & $0.404(2)$ & $-0.0974(12)$ & $5.7(4)$ \\
\hline $\mathrm{C}(58)$ & $0.881(2)$ & $0.266(2)$ & $-0.103(1)$ & $6.7(5)$ \\
\hline$C(59)$ & $0.856(5)$ & $0.206(4)$ & $-0.143(2)$ & $10.1(12)$ \\
\hline$C(60)$ & $0.744(4)$ & $0.282(4)$ & $-0.189(2)$ & $10.2(10)$ \\
\hline $\mathrm{C}(61)$ & $0.660(3)$ & $0.416(4)$ & $-0.1928(13)$ & $10.0(9)$ \\
\hline$C(62)$ & $0.683(2)$ & $0.474(2)$ & $-0.1456(10)$ & $6.6(5)$ \\
\hline $\mathrm{C}(63)$ & $0.5969(13)$ & $0.9501(12)$ & $-0.1306(7)$ & $4.0(2)$ \\
\hline$C(64)$ & $0.568(1)$ & $1.074(2)$ & $-0.1277(12)$ & $5.2(4)$ \\
\hline$C(65)$ & $0.482(2)$ & $1.190(2)$ & $-0.1799(12)$ & $6.1(4)$ \\
\hline$C(66)$ & $0.425(2)$ & $1.182(2)$ & $-0.2381(12)$ & $7.2(4)$ \\
\hline $\mathrm{C}(67)$ & $0.455(2)$ & $1.060(2)$ & $-0.2460(10)$ & $7.0(4)$ \\
\hline $\mathrm{C}(68)$ & $0.535(2)$ & $0.948(2)$ & $-0.1924(10)$ & $6.1(4)$ \\
\hline $\mathrm{C}(69)$ & $0.7901(13)$ & $0.8204(12)$ & $0.0074(8)$ & $4.3(3)$ \\
\hline $\mathrm{C}(70)$ & $0.783(2)$ & $0.766(2)$ & $0.0903(13)$ & $6.1(5)$ \\
\hline $\mathrm{C}(71)$ & $0.836(3)$ & $0.798(3)$ & $0.143(2)$ & $8.6(7)$ \\
\hline $\mathrm{C}(72)$ & $0.905(3)$ & $0.859(3)$ & $0.117(2)$ & $9.2(8)$ \\
\hline $\mathrm{C}(73)$ & $0.919(3)$ & $0.907(3)$ & $0.036(2)$ & $10.4(9)$ \\
\hline $\mathrm{C}(74)$ & $0.860(2)$ & $0.887(2)$ & $-0.0167(11)$ & $6.2(4)$ \\
\hline$C(75)$ & $0.308(2)$ & $0.778(2)$ & $0.100(1)$ & $8.0(5)$ \\
\hline $\mathrm{C}(76)$ & $0.374(4)$ & $0.719(4)$ & $0.174(2)$ & $12.9(9)$ \\
\hline $\mathrm{C}(77)$ & $0.346(4)$ & $0.972(4)$ & $0.025(3)$ & 12.6(9) \\
\hline $\mathrm{C}(78)$ & $0.413(5)$ & $0.983(5)$ & $0.077(3)$ & $14.1(12)$ \\
\hline $\mathrm{C}(79)$ & $0.286(6)$ & $0.887(7)$ & $-0.063(4)$ & 19.1(19) \\
\hline$C(80)$ & $0.266(7)$ & $0.804(7)$ & $-0.079(5)$ & $20.1(22)$ \\
\hline
\end{tabular}

$\mathrm{B}_{\mathrm{eq}}=8 / 3 \pi^{2}\left(\mathrm{U}_{11}\left(\mathrm{aa}^{*}\right)^{2}+\mathrm{U}_{22}\left(\mathrm{bb}^{*}\right)^{2}+\mathrm{U}_{33}\left(\mathrm{cc}^{*}\right)^{2}+2 \mathrm{U}_{12}\left(\mathrm{aa}^{*} \mathrm{bb}^{*}\right) \cos \gamma+2 \mathrm{U}_{13}(\mathrm{aa} * \mathrm{cc} *) \cos \beta+\right.$ $\left.2 \mathrm{U}_{23}\left(\mathrm{bb}^{*} \mathrm{cc} *\right) \cos \alpha\right)$

Table 2. Atomic coordinates and B iso involving hydrogens/Beq

$\begin{array}{lllll}\text { atom } & \mathrm{x} & \mathrm{y} & \mathrm{z} & \mathrm{B} e \mathrm{e} \\ \mathrm{H}(1) & 0.165(2) & 0.377(2) & -0.4233(11) & 8.1(6) \\ \mathrm{H}(2) & 0.058(2) & 0.335(3) & -0.306(2) & 10.2(8) \\ \mathrm{H}(3) & 0.076(4) & 0.122(5) & -0.264(2) & 16.0(18) \\ \mathrm{H}(4) & 0.214(2) & -0.046(2) & -0.3216(12) & 8.9(6) \\ \mathrm{H}(5) & 0.312(2) & 0.006(2) & -0.4406(10) & 7.7(5) \\ \mathrm{H}(6) & 0.202(2) & 0.099(2) & -0.580(2) & 8.7(7) \\ \mathrm{H}(7) & 0.278(5) & -0.062(2) & -0.654(2) & 12.9(16) \\ \mathrm{H}(8) & 0.468(4) & -0.133(3) & -0.715(2) & 12.3(11) \\ \mathrm{H}(9) & 0.584(3) & -0.029(2) & -0.726(1) & 10.6(8) \\ \mathrm{H}(10) & 0.542(2) & 0.108(2) & -0.6427(10) & 7.3(5) \\ \mathrm{H}(11) & 0.566(1) & 0.608(1) & -0.5897(10) & 6.1(4) \\ \mathrm{H}(12) & 0.709(2) & 0.647(2) & -0.6734(12) & 8.2(5) \\ \mathrm{H}(13) & 0.800(2) & 0.545(2) & -0.7751(10) & 7.4(5) \\ \mathrm{H}(14) & 0.757(2) & 0.384(2) & -0.7853(11) & 8.2(6) \\ \mathrm{H}(15) & 0.613(2) & 0.336(2) & -0.6997(10) & 7.2(5)\end{array}$




\begin{tabular}{|c|c|c|c|c|}
\hline $\mathrm{H}(16)$ & $0.422(3)$ & $0.372(2)$ & $-0.3921(13)$ & $7.9(6)$ \\
\hline $\mathrm{H}(17)$ & $0.340(3)$ & $0.509(2)$ & $-0.303(2)$ & $8.9(7)$ \\
\hline $\mathrm{H}(18)$ & $0.232(4)$ & $0.732(4)$ & $-0.348(2)$ & $16.9(23)$ \\
\hline $\mathrm{H}(19)$ & $0.197(2)$ & $0.837(2)$ & $-0.483(2)$ & $10.8(9)$ \\
\hline $\mathrm{H}(20)$ & $0.299(2)$ & $0.701(2)$ & $-0.5693(11)$ & $6.9(4)$ \\
\hline $\mathrm{H}(21)$ & $0.987(2)$ & $0.467(2)$ & $0.0766(12)$ & $7.8(6)$ \\
\hline $\mathrm{H}(22)$ & $1.107(2)$ & $0.309(4)$ & $0.193(2)$ & $11.3(10)$ \\
\hline $\mathrm{H}(23)$ & $1.094(4)$ & $0.105(3)$ & $0.236(2)$ & $11.8(10)$ \\
\hline $\mathrm{H}(24)$ & $0.956(2)$ & $0.078(2)$ & $0.1742(12)$ & $8.8(6)$ \\
\hline $\mathrm{H}(25)$ & $0.855(2)$ & $0.232(1)$ & $0.0595(9)$ & $6.4(4)$ \\
\hline $\mathrm{H}(26)$ & $0.964(2)$ & $0.224(2)$ & $-0.079(1)$ & $8.5(7)$ \\
\hline $\mathrm{H}(27)$ & $0.913(5)$ & $0.113(4)$ & $-0.139(2)$ & $14.6(20)$ \\
\hline $\mathrm{H}(28)$ & $0.724(4)$ & $0.240(4)$ & $-0.218(2)$ & $15.9(18)$ \\
\hline $\mathrm{H}(29)$ & $0.585(3)$ & $0.464(4)$ & $-0.2268(13)$ & $15.0(16)$ \\
\hline $\mathrm{H}(30)$ & $0.629(2)$ & $0.565(2)$ & $-0.1469(10)$ & $8.8(6)$ \\
\hline $\mathrm{H}(31)$ & $0.612(1)$ & $1.082(2)$ & $-0.0905(12)$ & $6.5(5)$ \\
\hline $\mathrm{H}(32)$ & $0.464(2)$ & $1.273(2)$ & $-0.1745(12)$ & $6.8(4)$ \\
\hline $\mathrm{H}(33)$ & $0.367(2)$ & $1.262(2)$ & $-0.2733(12)$ & $7.0(4)$ \\
\hline $\mathrm{H}(34)$ & $0.415(2)$ & $1.055(2)$ & $-0.2865(10)$ & $7.6(5)$ \\
\hline $\mathrm{H}(35)$ & $0.552(2)$ & $0.864(2)$ & $-0.1965(10)$ & $7.7(5)$ \\
\hline $\mathrm{H}(36)$ & $0.745(2)$ & $0.711(2)$ & $0.1095(13)$ & $7.7(6)$ \\
\hline $\mathrm{H}(37)$ & $0.821(3)$ & $0.775(3)$ & $0.197(2)$ & $10.4(9)$ \\
\hline $\mathrm{H}(38)$ & $0.940(3)$ & $0.876(3)$ & $0.155(2)$ & $11.6(11)$ \\
\hline $\mathrm{H}(39)$ & $0.976(3)$ & $0.943(3)$ & $0.018(2)$ & $14.3(12)$ \\
\hline $\mathrm{H}(40)$ & $0.863(2)$ & $0.926(2)$ & $-0.0706(11)$ & $7.9(5)$ \\
\hline $\mathrm{H}(41)$ & $0.267(1)$ & $0.556(1)$ & $-0.6405(9)$ & $5.9(4)$ \\
\hline $\mathrm{H}(42)$ & $0.359(1)$ & $0.435(1)$ & $-0.6749(9)$ & $5.9(4)$ \\
\hline $\mathrm{H}(43)$ & $0.810(1)$ & $0.713(1)$ & $-0.1762(9)$ & $5.8(4)$ \\
\hline $\mathrm{H}(44)$ & $0.902(1)$ & $0.742(1)$ & $-0.1418(9)$ & $5.8(4)$ \\
\hline $\mathrm{H}(45)$ & $0.872(2)$ & $-0.072(2)$ & $-0.4158(13)$ & $8.8(5)$ \\
\hline $\mathrm{H}(46)$ & $0.941(2)$ & $-0.004(2)$ & $-0.3966(13)$ & $8.8(5)$ \\
\hline $\mathrm{H}(49)$ & $0.920(6)$ & $0.176(6)$ & $-0.470(4)$ & $21.6(20)$ \\
\hline $\mathrm{H}(50)$ & $0.813(6)$ & $0.284(6)$ & $-0.532(4)$ & $21.6(20)$ \\
\hline $\mathrm{H}(51)$ & $0.361(4)$ & $1.016(4)$ & $-0.025(3)$ & $15.1(11)$ \\
\hline $\mathrm{H}(52)$ & $0.253(4)$ & $1.011(4)$ & $0.035(3)$ & 15.1(11) \\
\hline $\mathrm{H}(53)$ & $0.204(6)$ & $0.954(7)$ & $-0.049(4)$ & $22.9(23)$ \\
\hline $\mathrm{H}(54)$ & $0.324(6)$ & $0.926(7)$ & $-0.105(4)$ & $22.9(23)$ \\
\hline $\mathrm{H}(55)$ & $0.241(2)$ & $0.862(2)$ & $0.108(1)$ & $9.6(6)$ \\
\hline $\mathrm{H}(56)$ & $0.268(2)$ & $0.738(2)$ & $0.083(1)$ & $9.6(6)$ \\
\hline $\mathrm{H}(57)$ & $0.170(2)$ & $0.379(3)$ & $-0.722(2)$ & $12.5(10)$ \\
\hline $\mathrm{H}(58)$ & $0.039(2)$ & $0.514(3)$ & $-0.737(2)$ & $12.5(10)$ \\
\hline $\mathrm{H}(59)$ & $0.173(2)$ & $0.511(3)$ & $-0.742(2)$ & $12.5(10)$ \\
\hline $\mathrm{H}(60)$ & $0.069(2)$ & $0.658(2)$ & $-0.653(2)$ & $10.0(8)$ \\
\hline $\mathrm{H}(61)$ & $-0.056(2)$ & $0.644(2)$ & $-0.645(2)$ & $10.0(8)$ \\
\hline $\mathrm{H}(62)$ & $0.019(2)$ & $0.612(2)$ & $-0.571(2)$ & $10.0(8)$ \\
\hline
\end{tabular}




\begin{tabular}{|c|c|c|c|c|}
\hline $\mathrm{H}(63)$ & $0.028(2)$ & $0.386(3)$ & $-0.536(2)$ & 11.7(9) \\
\hline $\mathrm{H}(64)$ & $-0.048(2)$ & $0.441(3)$ & $-0.614(2)$ & 11.7(9) \\
\hline $\mathrm{H}(65)$ & $0.083(2)$ & $0.305(3)$ & $-0.599(2)$ & 11.7(9) \\
\hline $\mathrm{H}(66)$ & $0.571(7)$ & $-0.256(4)$ & $-0.325(4)$ & $24.9(33)$ \\
\hline $\mathrm{H}(67)$ & $0.510(7)$ & $-0.212(4)$ & $-0.407(4)$ & $24.9(33)$ \\
\hline $\mathrm{H}(68)$ & $0.611(7)$ & $-0.361(4)$ & $-0.372(4)$ & $24.9(33)$ \\
\hline $\mathrm{H}(69)$ & $0.575(7)$ & $-0.228(3)$ & $-0.515(2)$ & $26.4(39)$ \\
\hline $\mathrm{H}(70)$ & $0.726(7)$ & $-0.278(3)$ & $-0.524(2)$ & $26.4(39)$ \\
\hline $\mathrm{H}(71)$ & $0.677(7)$ & $-0.376(3)$ & $-0.480(2)$ & $26.4(39)$ \\
\hline $\mathrm{H}(72)$ & $0.826(6)$ & $-0.423(3)$ & $-0.384(6)$ & $38.5(66)$ \\
\hline $\mathrm{H}(73)$ & $0.856(6)$ & $-0.313(3)$ & $-0.436(6)$ & $38.5(66)$ \\
\hline $\mathrm{H}(74)$ & $0.797(6)$ & $-0.303(3)$ & $-0.353(6)$ & $38.5(66)$ \\
\hline $\mathrm{H}(75)$ & $0.783(4)$ & $0.373(4)$ & $-0.421(3)$ & $16.6(13)$ \\
\hline $\mathrm{H}(76)$ & $0.661(4)$ & $0.365(4)$ & $-0.435(3)$ & $16.6(13)$ \\
\hline $\mathrm{H}(77)$ & $0.766(4)$ & $0.254(4)$ & $-0.372(3)$ & $16.6(13)$ \\
\hline $\mathrm{H}(78)$ & $0.779(4)$ & $0.078(4)$ & $-0.313(2)$ & $15.1(11)$ \\
\hline $\mathrm{H}(79)$ & $0.707(4)$ & $0.014(4)$ & $-0.334(2)$ & $15.1(11)$ \\
\hline $\mathrm{H}(80)$ & $0.839(4)$ & $-0.075(4)$ & $-0.288(2)$ & $15.1(11)$ \\
\hline $\mathrm{H}(84)$ & $1.080(2)$ & $0.304(2)$ & $-0.088(2)$ & $10.8(9)$ \\
\hline $\mathrm{H}(85)$ & $1.212(2)$ & $0.303(2)$ & $-0.112(2)$ & $10.8(9)$ \\
\hline $\mathrm{H}(86)$ & $1.145(2)$ & $0.337(2)$ & $-0.035(2)$ & $10.8(9)$ \\
\hline $\mathrm{H}(87)$ & $1.004(3)$ & $0.602(3)$ & $-0.2403(13)$ & $11.4(10)$ \\
\hline $\mathrm{H}(88)$ & $1.128(3)$ & $0.464(3)$ & $-0.2368(13)$ & $11.4(10)$ \\
\hline $\mathrm{H}(89)$ & $0.989(3)$ & $0.477(3)$ & $-0.2236(13)$ & $11.4(10)$ \\
\hline $\mathrm{H}(90)$ & $1.154(2)$ & $0.538(3)$ & $-0.069(2)$ & $10.9(9)$ \\
\hline $\mathrm{H}(91)$ & $1.225(2)$ & $0.509(3)$ & $-0.146(2)$ & $10.9(9)$ \\
\hline $\mathrm{H}(92)$ & $1.096(2)$ & $0.642(3)$ & $-0.147(2)$ & $10.9(9)$ \\
\hline $\mathrm{H}(93)$ & $0.419(6)$ & $0.380(6)$ & $-0.021(4)$ & $22.6(22)$ \\
\hline $\mathrm{H}(94)$ & $0.569(6)$ & $0.280(6)$ & $-0.024(4)$ & $22.6(22)$ \\
\hline $\mathrm{H}(95)$ & $0.472(6)$ & $0.229(6)$ & $0.015(4)$ & $22.6(22)$ \\
\hline $\mathrm{H}(96)$ & $0.571(7)$ & $0.154(7)$ & $0.131(5)$ & $25.6(27)$ \\
\hline $\mathrm{H}(97)$ & $0.669(7)$ & $0.197(7)$ & $0.083(5)$ & $25.6(27)$ \\
\hline $\mathrm{H}(98)$ & $0.623(7)$ & $0.224(7)$ & $0.165(5)$ & $25.6(27)$ \\
\hline H(99) & $0.367(5)$ & $0.409(6)$ & $0.156(3)$ & $20.9(20)$ \\
\hline $\mathrm{H}(100)$ & $0.317(5)$ & $0.455(6)$ & $0.071(3)$ & $20.9(20)$ \\
\hline $\mathrm{H}(101)$ & $0.339(5)$ & $0.319(6)$ & $0.124(3)$ & $20.9(20)$ \\
\hline $\mathrm{H}(102)$ & $0.315(4)$ & $0.707(4)$ & $0.213(2)$ & $15.5(11)$ \\
\hline $\mathrm{H}(103)$ & $0.441(4)$ & $0.635(4)$ & $0.166(2)$ & $15.5(11)$ \\
\hline H(104) & $0.414(4)$ & $0.759(4)$ & $0.190(2)$ & $15.5(11)$ \\
\hline $\mathrm{H}(105)$ & $0.398(5)$ & $0.939(5)$ & $0.127(3)$ & $17.0(14)$ \\
\hline H(106) & $0.506(5)$ & $0.943(5)$ & $0.068(3)$ & $17.0(14)$ \\
\hline H(107) & $0.379(5)$ & $1.074(5)$ & $0.076(3)$ & $17.0(14)$ \\
\hline $\mathrm{H}(108)$ & $0.229(7)$ & $0.766(7)$ & $-0.037(5)$ & $24.1(26)$ \\
\hline H(109) & $0.209(7)$ & $0.845(7)$ & $-0.123(5)$ & $24.1(26)$ \\
\hline $\mathrm{H}(110)$ & $0.350(7)$ & $0.737(7)$ & $-0.092(5)$ & $24.1(26)$ \\
\hline
\end{tabular}




$\begin{array}{llrrr}\mathrm{H}(47) & 0.903(6) & 0.108(6) & -0.566(4) & 25.0(23) \\ \mathrm{H}(48) & 0.954(6) & 0.002(6) & -0.487(4) & 25.0(23) \\ \mathrm{H}(81) & 0.883(5) & -0.103(5) & -0.530(3) & 20.4(16) \\ \mathrm{H}(82) & 0.827(5) & 0.004(5) & -0.607(3) & 20.4(16) \\ \mathrm{H}(83) & 0.978(5) & -0.078(5) & -0.592(3) & 20.4(16)\end{array}$

$B_{e q}=8 / 3 \pi^{2}\left(U_{11}\left(a a^{*}\right)^{2}+U_{22}\left(b^{*}\right)^{2}+U_{33}\left(c c^{*}\right)^{2}+2 U_{12}\left(a a^{*} b b^{*}\right) \cos \gamma+2 U_{13}\left(a a^{*} c c^{*}\right) \cos \beta+\right.$ $\left.2 \mathrm{U}_{23}\left(\mathrm{bb}^{*} \mathrm{cc} *\right) \cos \alpha\right)$

Table 3. Anisotropic Displacement Parameters

\begin{tabular}{|c|c|c|c|c|c|c|}
\hline atom & $\mathrm{U}_{11}$ & $\mathrm{U}_{22}$ & U33 & $\mathrm{U}_{12}$ & $\mathrm{U}_{13}$ & $\mathrm{U}_{23}$ \\
\hline $\operatorname{Pt}(1)$ & $0.0479(3)$ & $0.0407(3)$ & $0.0601(5)$ & $-0.0219(3)$ & $-0.0037(3)$ & $-0.01(0)$ \\
\hline $\operatorname{Pt}(2)$ & $0.0477(3)$ & $0.0449(4)$ & $0.0567(5)$ & $-0.0260(3)$ & $0.0019(3)$ & $-0.02(-1)$ \\
\hline $\mathrm{P}(1)$ & $0.067(2)$ & $0.113(4)$ & $0.118(4)$ & $-0.049(3)$ & $-0.026(3)$ & $-0.02(-1)$ \\
\hline $\mathrm{P}(2)$ & $0.073(3)$ & $0.084(3)$ & $0.126(4)$ & $-0.023(2)$ & $0.028(3)$ & $-0.06(-5)$ \\
\hline $\mathrm{Si}(1)$ & $0.050(2)$ & $0.045(2)$ & $0.061(2)$ & $-0.026(1)$ & $-0.004(1)$ & $-0.02(-1)$ \\
\hline $\operatorname{Si}(2)$ & $0.056(2)$ & $0.050(2)$ & $0.059(2)$ & $-0.033(2)$ & $0.002(1)$ & $-0.02(-1)$ \\
\hline $\operatorname{Si}(3)$ & $0.051(2)$ & $0.046(2)$ & $0.059(2)$ & $-0.025(1)$ & $-0.000(1)$ & $-0.02(-1)$ \\
\hline $\operatorname{Si}(4)$ & $0.055(2)$ & $0.041(2)$ & $0.059(2)$ & $-0.025(1)$ & $-0.002(1)$ & $-0.01(0)$ \\
\hline $\mathrm{N}(1)$ & $0.046(5)$ & $0.058(7)$ & $0.071(8)$ & $-0.020(5)$ & $-0.015(6)$ & $-0.00(0)$ \\
\hline $\mathrm{N}(2)$ & $0.105(10)$ & $0.034(6)$ & $0.070(8)$ & $-0.022(6)$ & $-0.023(7)$ & $-0.01(0)$ \\
\hline $\mathrm{N}(3)$ & $0.054(6)$ & $0.079(8)$ & $0.070(8)$ & $-0.034(6)$ & $0.012(6)$ & $-0.02(-1)$ \\
\hline $\mathrm{N}(4)$ & $0.092(8)$ & $0.080(8)$ & $0.075(8)$ & $-0.069(7)$ & $0.019(6)$ & $-0.03(-2)$ \\
\hline $\mathrm{C}(1)$ & $0.064(8)$ & $0.054(7)$ & $0.069(8)$ & $-0.030(6)$ & $-0.007(6)$ & $-0.01(0)$ \\
\hline $\mathrm{C}(2)$ & $0.050(7)$ & $0.067(9)$ & $0.080(11)$ & $-0.020(7)$ & $-0.014(7)$ & $-0.01(0)$ \\
\hline $\mathrm{C}(3)$ & $0.09(1)$ & $0.16(2)$ & $0.14(2)$ & $-0.04(2)$ & $-0.06(2)$ & $-0.05(-4)$ \\
\hline $\mathrm{C}(4)$ & $0.062(10)$ & $0.078(13)$ & $0.18(3)$ & $-0.006(9)$ & $-0.042(13)$ & $0.013(13)$ \\
\hline$C(5)$ & $0.075(11)$ & $0.13(2)$ & $0.17(2)$ & $-0.057(12)$ & $-0.06(1)$ & $0.012(12)$ \\
\hline $\mathrm{C}(6)$ & $0.083(9)$ & $0.050(8)$ & $0.066(8)$ & $-0.034(7)$ & $0.006(7)$ & $-0.03(-2)$ \\
\hline $\mathrm{C}(7)$ & $0.11(1)$ & $0.031(7)$ & $0.083(11)$ & $-0.025(8)$ & $-0.015(10)$ & $-0.01(0)$ \\
\hline $\mathrm{C}(8)$ & $0.41(9)$ & $0.06(2)$ & $0.31(6)$ & $-0.03(4)$ & $0.16(6)$ & $-0.03(-2)$ \\
\hline $\mathrm{C}(9)$ & $0.58(11)$ & $0.08(2)$ & $0.17(3)$ & $-0.11(4)$ & $-0.19(5)$ & $-0.01(0)$ \\
\hline $\mathrm{C}(10)$ & $0.26(5)$ & $0.033(13)$ & $0.9(2)$ & $-0.00(2)$ & $-0.35(9)$ & $-0.04(-3)$ \\
\hline $\mathrm{C}(11)$ & $0.061(7)$ & $0.067(8)$ & $0.064(8)$ & $-0.033(6)$ & $-0.010(6)$ & $-0.01(0)$ \\
\hline $\mathrm{C}(12)$ & $0.11(1)$ & $0.086(12)$ & $0.062(10)$ & $-0.053(12)$ & $0.009(11)$ & $-0.03(-2)$ \\
\hline $\mathrm{C}(13)$ & $0.09(1)$ & $0.14(2)$ & $0.10(2)$ & $-0.04(2)$ & $0.023(12)$ & $-0.04(-3)$ \\
\hline $\mathrm{C}(14)$ & $0.17(3)$ & $0.26(4)$ & $0.07(1)$ & $-0.15(3)$ & $0.05(2)$ & $-0.05(-4)$ \\
\hline $\mathrm{C}(15)$ & $0.097(13)$ & $0.10(1)$ & $0.080(12)$ & $-0.050(11)$ & $0.017(10)$ & $-0.01(0)$ \\
\hline$C(16)$ & $0.078(9)$ & $0.088(11)$ & $0.076(10)$ & $-0.057(9)$ & $0.010(8)$ & $-0.02(-1)$ \\
\hline $\mathrm{C}(17)$ & $0.12(2)$ & $0.068(11)$ & $0.056(9)$ & $-0.055(11)$ & $-0.016(9)$ & $-0.01(0)$ \\
\hline $\mathrm{C}(18)$ & $0.089(13)$ & $0.060(9)$ & $0.13(2)$ & $-0.035(9)$ & $-0.042(12)$ & $-0.02(-1)$ \\
\hline $\mathrm{C}(19)$ & $0.22(4)$ & $0.048(12)$ & $0.15(3)$ & $-0.05(2)$ & $-0.11(3)$ & $-0.00(0)$ \\
\hline $\mathrm{C}(20)$ & $0.21(3)$ & $0.07(1)$ & $0.11(2)$ & $-0.03(2)$ & $-0.06(2)$ & $-0.04(-3)$ \\
\hline $\mathrm{C}(21)$ & $0.16(2)$ & $0.082(12)$ & $0.09(1)$ & $-0.04(1)$ & $-0.019(13)$ & $-0.04(-3)$ \\
\hline $\mathrm{C}(22)$ & $0.097(12)$ & $0.068(9)$ & $0.068(9)$ & $-0.034(8)$ & $-0.002(8)$ & $-0.02(-1)$ \\
\hline
\end{tabular}




\begin{tabular}{|c|c|c|c|c|c|c|}
\hline$C(23)$ & $0.063(7)$ & $0.062(7)$ & $0.052(7)$ & $-0.043(6)$ & $-0.000(5)$ & $-0.01(0)$ \\
\hline$C(24)$ & $0.053(7)$ & $0.052(7)$ & $0.089(10)$ & $-0.026(6)$ & $-0.005(7)$ & $-0.02(-1)$ \\
\hline$C(25)$ & $0.074(9)$ & $0.087(11)$ & $0.100(13)$ & $-0.053(9)$ & $0.001(9)$ & $-0.01(0)$ \\
\hline$C(26)$ & $0.055(8)$ & $0.108(13)$ & $0.073(10)$ & $-0.042(8)$ & $0.008(7)$ & $0.007(7)$ \\
\hline $\mathrm{C}(27)$ & $0.087(11)$ & $0.105(13)$ & $0.067(10)$ & $-0.049(10)$ & $0.019(8)$ & $-0.03(-2)$ \\
\hline $\mathrm{C}(28)$ & $0.077(9)$ & $0.078(10)$ & $0.071(9)$ & $-0.039(8)$ & $0.016(7)$ & $-0.03(-2)$ \\
\hline $\mathrm{C}(29)$ & $0.054(6)$ & $0.045(6)$ & $0.062(7)$ & $-0.024(5)$ & $0.016(5)$ & $-0.02(-1)$ \\
\hline $\mathrm{C}(30)$ & $0.12(2)$ & $0.068(11)$ & $0.063(10)$ & $-0.041(11)$ & $0.013(10)$ & $-0.03(-2)$ \\
\hline $\mathrm{C}(31)$ & $0.12(2)$ & $0.09(1)$ & $0.08(1)$ & $-0.034(13)$ & $0.015(12)$ & $-0.02(-1)$ \\
\hline $\mathrm{C}(32)$ & $0.20(3)$ & $0.16(3)$ & $0.18(3)$ & $-0.13(3)$ & $0.13(3)$ & $-0.14(-13)$ \\
\hline $\mathrm{C}(33)$ & $0.10(1)$ & $0.069(11)$ & $0.17(3)$ & $-0.035(10)$ & $0.05(2)$ & $-0.05(-4)$ \\
\hline $\mathrm{C}(34)$ & $0.079(9)$ & $0.055(8)$ & $0.085(11)$ & $-0.021(7)$ & $-0.003(8)$ & $-0.02(-1)$ \\
\hline $\mathrm{C}(41)$ & $0.063(7)$ & $0.059(7)$ & $0.061(8)$ & $-0.034(6)$ & $0.010(6)$ & $-0.01(0)$ \\
\hline $\mathrm{C}(42)$ & $0.046(6)$ & $0.085(11)$ & $0.076(10)$ & $-0.028(7)$ & $0.025(7)$ & $-0.04(-3)$ \\
\hline $\mathrm{C}(43)$ & $0.089(12)$ & $0.064(11)$ & $0.19(3)$ & $-0.029(9)$ & $0.08(2)$ & $-0.04(-3)$ \\
\hline$C(44)$ & $0.11(2)$ & $0.16(2)$ & $0.09(1)$ & $-0.05(2)$ & $0.040(12)$ & $-0.07(-6)$ \\
\hline$C(45)$ & $0.050(8)$ & $0.13(2)$ & $0.16(2)$ & $-0.047(11)$ & $0.000(11)$ & $-0.03(-2)$ \\
\hline$C(46)$ & $0.080(10)$ & $0.099(12)$ & $0.067(9)$ & $-0.064(9)$ & $0.016(7)$ & $-0.03(-2)$ \\
\hline $\mathrm{C}(51)$ & $0.060(7)$ & $0.064(8)$ & $0.064(8)$ & $-0.030(6)$ & $0.010(6)$ & $-0.03(-2)$ \\
\hline$C(52)$ & $0.103(13)$ & $0.073(11)$ & $0.073(11)$ & $-0.040(10)$ & $-0.019(11)$ & $-0.02(-1)$ \\
\hline$C(53)$ & $0.070(12)$ & $0.18(3)$ & $0.11(2)$ & $-0.02(2)$ & $-0.027(12)$ & $-0.07(-6)$ \\
\hline$C(54)$ & $0.20(3)$ & $0.10(2)$ & $0.07(1)$ & $-0.06(2)$ & $-0.04(2)$ & $0.03(3)$ \\
\hline$C(55)$ & $0.11(2)$ & $0.10(1)$ & $0.070(11)$ & $-0.047(12)$ & $-0.018(10)$ & $0.012(12)$ \\
\hline$C(56)$ & $0.076(9)$ & $0.058(8)$ & $0.068(9)$ & $-0.027(7)$ & $-0.004(7)$ & $-0.01(0)$ \\
\hline $\mathrm{C}(57)$ & $0.096(12)$ & $0.072(11)$ & $0.069(11)$ & $-0.050(10)$ & $0.015(9)$ & $-0.04(-3)$ \\
\hline $\mathrm{C}(58)$ & $0.089(13)$ & $0.081(12)$ & $0.10(1)$ & $-0.051(10)$ & $0.041(11)$ & $-0.05(-4)$ \\
\hline$C(59)$ & $0.20(4)$ & $0.15(3)$ & $0.10(2)$ & $-0.14(3)$ & $0.06(2)$ & $-0.06(-5)$ \\
\hline$C(60)$ & $0.24(4)$ & $0.17(3)$ & $0.09(2)$ & $-0.18(3)$ & $0.07(2)$ & $-0.08(-7)$ \\
\hline$C(61)$ & $0.20(3)$ & $0.21(3)$ & $0.069(12)$ & $-0.17(3)$ & $0.01(1)$ & $-0.04(-3)$ \\
\hline$C(62)$ & $0.12(1)$ & $0.104(13)$ & $0.054(8)$ & $-0.073(12)$ & $-0.001(8)$ & $-0.02(-1)$ \\
\hline $\mathrm{C}(63)$ & $0.061(7)$ & $0.040(6)$ & $0.046(6)$ & $-0.022(5)$ & $-0.005(5)$ & $-0.01(0)$ \\
\hline$C(64)$ & $0.054(7)$ & $0.050(8)$ & $0.103(13)$ & $-0.024(6)$ & $-0.001(8)$ & $-0.03(-2)$ \\
\hline $\mathrm{C}(65)$ & $0.065(8)$ & $0.054(9)$ & $0.096(12)$ & $-0.019(7)$ & $0.005(8)$ & $-0.02(-1)$ \\
\hline$C(66)$ & $0.049(7)$ & $0.089(12)$ & $0.082(11)$ & $-0.013(8)$ & $0.004(7)$ & $0.007(7)$ \\
\hline$C(67)$ & $0.081(11)$ & $0.093(13)$ & $0.065(10)$ & $-0.022(9)$ & $-0.025(8)$ & $-0.01(0)$ \\
\hline $\mathrm{C}(68)$ & $0.090(10)$ & $0.087(10)$ & $0.068(9)$ & $-0.048(9)$ & $-0.022(8)$ & $-0.02(-1)$ \\
\hline $\mathrm{C}(69)$ & $0.058(6)$ & $0.045(6)$ & $0.062(7)$ & $-0.027(5)$ & $-0.013(5)$ & $-0.01(0)$ \\
\hline $\mathrm{C}(70)$ & $0.092(12)$ & $0.081(13)$ & $0.071(11)$ & $-0.053(11)$ & $-0.033(9)$ & $-0.00(0)$ \\
\hline $\mathrm{C}(71)$ & $0.12(2)$ & $0.10(2)$ & $0.11(2)$ & $-0.04(2)$ & $-0.03(1)$ & $-0.03(-2)$ \\
\hline $\mathrm{C}(72)$ & $0.14(2)$ & $0.11(2)$ & $0.12(2)$ & $-0.07(2)$ & $-0.08(2)$ & $-0.01(0)$ \\
\hline $\mathrm{C}(73)$ & $0.12(2)$ & $0.12(2)$ & $0.21(3)$ & $-0.09(2)$ & $-0.04(2)$ & $-0.03(-2)$ \\
\hline $\mathrm{C}(74)$ & $0.078(9)$ & $0.086(10)$ & $0.087(11)$ & $-0.058(8)$ & $-0.008(8)$ & $-0.01(0)$ \\
\hline
\end{tabular}

The general temperature factor expression: $\exp \left(-2 \pi^{2}\left(\mathrm{a}^{* 2} \mathrm{U} 1 \mathrm{~h}^{2}+\mathrm{b}^{* 2} \mathrm{U}_{22} \mathrm{k}^{2}+\mathrm{c}^{* 2} \mathrm{U}_{33 \mathrm{I}^{2}+}\right.\right.$ $\left.\left.2 \mathrm{a} * \mathrm{~b} * \mathrm{U}_{12} \mathrm{hk}+2 \mathrm{a} * \mathrm{c} * \mathrm{U}_{13} \mathrm{hl}+2 \mathrm{~b} * \mathrm{c} * \mathrm{U}_{23} \mathrm{kl}\right)\right)$ 
Table 4. Bond lengths ( $)$

\begin{tabular}{|c|c|c|c|c|c|}
\hline atom & atom & distance & atom & atom & distance \\
\hline $\operatorname{Pt}(1)$ & $\mathrm{P}(1)$ & $2.392(8)$ & $\operatorname{Pt}(1)$ & $\operatorname{Si}(1)$ & $2.333(6)$ \\
\hline $\operatorname{Pt}(1)$ & $\operatorname{Si}(2)$ & $2.357(5)$ & $\operatorname{Pt}(1)$ & $C(6)$ & $1.97(2)$ \\
\hline $\operatorname{Pt}(2)$ & $\mathrm{P}(2)$ & $2.360(6)$ & $\operatorname{Pt}(2)$ & $\mathrm{Si}(3)$ & $2.382(5)$ \\
\hline $\operatorname{Pt}(2)$ & $\mathrm{Si}(4)$ & $2.370(7)$ & $\operatorname{Pt}(2)$ & $C(46)$ & $1.94(2)$ \\
\hline $\mathrm{P}(1)$ & $\mathrm{C}(35)$ & $1.73(2)$ & $\mathrm{P}(1)$ & $C(37)$ & $1.76(6)$ \\
\hline $\mathrm{P}(1)$ & $C(39)$ & $1.57(9)$ & $P(2)$ & $C(75)$ & $1.75(3)$ \\
\hline$P(2)$ & $\mathrm{C}(77)$ & $1.60(4)$ & $P(2)$ & $C(79)$ & $2.08(7)$ \\
\hline $\mathrm{Si}(1)$ & $\mathrm{N}(1)$ & $1.74(1)$ & $\mathrm{Si}(1)$ & $\mathrm{C}(11)$ & $1.90(2)$ \\
\hline $\mathrm{Si}(1)$ & $\mathrm{C}(17)$ & $1.89(3)$ & $\operatorname{Si}(2)$ & $\mathrm{C}(1)$ & $1.87(2)$ \\
\hline $\operatorname{Si}(2)$ & $\mathrm{C}(23)$ & $1.88(2)$ & $\mathrm{Si}(2)$ & $C(29)$ & $1.92(2)$ \\
\hline $\mathrm{Si}(3)$ & $\mathrm{N}(3)$ & $1.74(2)$ & $\mathrm{Si}(3)$ & $\mathrm{C}(51)$ & $1.90(1)$ \\
\hline $\mathrm{Si}(3)$ & $C(57)$ & $1.88(3)$ & $\mathrm{Si}(4)$ & $\mathrm{C}(41)$ & $1.89(1)$ \\
\hline $\mathrm{Si}(4)$ & $\mathrm{C}(63)$ & $1.87(1)$ & $\mathrm{Si}(4)$ & $C(69)$ & $1.92(2)$ \\
\hline $\mathrm{N}(1)$ & $\mathrm{C}(1)$ & $1.47(2)$ & $\mathrm{N}(1)$ & $\mathrm{C}(2)$ & $1.49(2)$ \\
\hline $\mathrm{N}(2)$ & $C(6)$ & $1.17(2)$ & $\mathrm{N}(2)$ & $C(7)$ & $1.45(2)$ \\
\hline $\mathrm{N}(3)$ & $\mathrm{C}(41)$ & $1.49(2)$ & $\mathrm{N}(3)$ & $\mathrm{C}(42)$ & $1.52(2)$ \\
\hline $\mathrm{N}(4)$ & $C(46)$ & $1.19(3)$ & $\mathrm{N}(4)$ & $\mathrm{C}(47)$ & $1.46(3)$ \\
\hline $\mathrm{C}(2)$ & $\mathrm{C}(3)$ & $1.54(4)$ & $\mathrm{C}(2)$ & $\mathrm{C}(4)$ & $1.58(3)$ \\
\hline $\mathrm{C}(2)$ & $C(5)$ & $1.47(4)$ & $\mathrm{C}(7)$ & $\mathrm{C}(8)$ & $1.21(8)$ \\
\hline $\mathrm{C}(7)$ & $\mathrm{C}(9)$ & $1.43(6)$ & $C(7)$ & $C(10)$ & $1.36(7)$ \\
\hline $\mathrm{C}(11)$ & $\mathrm{C}(12)$ & $1.41(3)$ & $\mathrm{C}(11)$ & $C(16)$ & $1.38(3)$ \\
\hline $\mathrm{C}(12)$ & $\mathrm{C}(13)$ & $1.38(3)$ & $\mathrm{C}(13)$ & $C(14)$ & $1.42(7)$ \\
\hline $\mathrm{C}(14)$ & $\mathrm{C}(15)$ & $1.33(5)$ & $C(15)$ & $C(16)$ & $1.40(3)$ \\
\hline $\mathrm{C}(17)$ & $\mathrm{C}(18)$ & $1.44(5)$ & $\mathrm{C}(17)$ & $C(22)$ & $1.42(3)$ \\
\hline $\mathrm{C}(18)$ & $\mathrm{C}(19)$ & $1.43(5)$ & $C(19)$ & $\mathrm{C}(20)$ & $1.27(6)$ \\
\hline $\mathrm{C}(20)$ & $\mathrm{C}(21)$ & $1.31(7)$ & $\mathrm{C}(21)$ & $C(22)$ & $1.39(4)$ \\
\hline $\mathrm{C}(23)$ & $\mathrm{C}(24)$ & $1.39(3)$ & $\mathrm{C}(23)$ & $\mathrm{C}(28)$ & $1.45(2)$ \\
\hline $\mathrm{C}(24)$ & $\mathrm{C}(25)$ & $1.34(3)$ & $\mathrm{C}(25)$ & $C(26)$ & $1.38(3)$ \\
\hline$C(26)$ & $\mathrm{C}(27)$ & $1.34(4)$ & $\mathrm{C}(27)$ & $\mathrm{C}(28)$ & $1.38(3)$ \\
\hline $\mathrm{C}(29)$ & $\mathrm{C}(30)$ & $1.44(2)$ & $\mathrm{C}(29)$ & $C(34)$ & $1.35(2)$ \\
\hline$C(30)$ & $\mathrm{C}(31)$ & $1.39(4)$ & $\mathrm{C}(31)$ & $C(32)$ & $1.39(4)$ \\
\hline $\mathrm{C}(32)$ & $\mathrm{C}(33)$ & $1.40(5)$ & $\mathrm{C}(33)$ & $C(34)$ & $1.37(4)$ \\
\hline$C(35)$ & $\mathrm{C}(36)$ & $1.43(4)$ & $\mathrm{C}(37)$ & $\mathrm{C}(38)$ & $1.4(1)$ \\
\hline $\mathrm{C}(39)$ & $\mathrm{C}(40)$ & $1.56(8)$ & $\mathrm{C}(42)$ & $\mathrm{C}(43)$ & $1.48(2)$ \\
\hline $\mathrm{C}(42)$ & $\mathrm{C}(44)$ & $1.51(3)$ & $\mathrm{C}(42)$ & $\mathrm{C}(45)$ & $1.57(4)$ \\
\hline $\mathrm{C}(47)$ & $\mathrm{C}(48)$ & $1.44(8)$ & $\mathrm{C}(47)$ & C(49) & $1.28(6)$ \\
\hline $\mathrm{C}(47)$ & $\mathrm{C}(50)$ & $1.46(6)$ & $\mathrm{C}(51)$ & $C(52)$ & $1.37(4)$ \\
\hline $\mathrm{C}(51)$ & $C(56)$ & $1.42(3)$ & $C(52)$ & $C(53)$ & $1.42(3)$ \\
\hline $\mathrm{C}(53)$ & $\mathrm{C}(54)$ & $1.45(6)$ & $\mathrm{C}(54)$ & $C(55)$ & $1.34(6)$ \\
\hline$C(55)$ & $C(56)$ & $1.39(2)$ & $C(57)$ & $\mathrm{C}(58)$ & $1.46(3)$ \\
\hline$C(57)$ & $\mathrm{C}(62)$ & $1.41(3)$ & $\mathrm{C}(58)$ & C(59) & $1.31(7)$ \\
\hline $\mathrm{C}(59)$ & $\mathrm{C}(60)$ & $1.38(6)$ & $C(60)$ & $\mathrm{C}(61)$ & $1.38(5)$ \\
\hline
\end{tabular}




$\begin{array}{llllll}\mathrm{C}(61) & \mathrm{C}(62) & 1.38(5) & \mathrm{C}(63) & \mathrm{C}(64) & 1.38(3) \\ \mathrm{C}(63) & \mathrm{C}(68) & 1.43(3) & \mathrm{C}(64) & \mathrm{C}(65) & 1.39(2) \\ \mathrm{C}(65) & \mathrm{C}(66) & 1.37(3) & \mathrm{C}(66) & \mathrm{C}(67) & 1.38(4) \\ \mathrm{C}(67) & \mathrm{C}(68) & 1.36(2) & \mathrm{C}(69) & \mathrm{C}(70) & 1.45(3) \\ \mathrm{C}(69) & \mathrm{C}(74) & 1.37(3) & \mathrm{C}(70) & \mathrm{C}(71) & 1.43(5) \\ \mathrm{C}(71) & \mathrm{C}(72) & 1.33(6) & \mathrm{C}(72) & \mathrm{C}(73) & 1.43(5) \\ \mathrm{C}(73) & \mathrm{C}(74) & 1.39(5) & \mathrm{C}(75) & \mathrm{C}(76) & 1.44(5) \\ \mathrm{C}(77) & \mathrm{C}(78) & 1.39(9) & \mathrm{C}(79) & \mathrm{C}(80) & 1.3(1)\end{array}$

Table 5. Bond lengths involving hydrogens $(\AA)$

\begin{tabular}{|c|c|c|c|c|c|}
\hline atom & atom & distance & atom & atom & distance \\
\hline $\mathrm{C}(1)$ & $\mathrm{H}(41)$ & $0.95(2)$ & $\mathrm{C}(1)$ & $\mathrm{H}(42)$ & $0.95(2)$ \\
\hline $\mathrm{C}(3)$ & $\mathrm{H}(57)$ & $0.95(5)$ & $\mathrm{C}(3)$ & $\mathrm{H}(58)$ & $0.95(4)$ \\
\hline $\mathrm{C}(3)$ & $\mathrm{H}(59)$ & $0.95(5)$ & $\mathrm{C}(4)$ & $\mathrm{H}(60)$ & $0.95(4)$ \\
\hline $\mathrm{C}(4)$ & $\mathrm{H}(61)$ & $0.95(4)$ & $\mathrm{C}(4)$ & $\mathrm{H}(62)$ & $0.95(5)$ \\
\hline$C(5)$ & $\mathrm{H}(63)$ & $0.95(5)$ & $C(5)$ & $\mathrm{H}(64)$ & $0.95(3)$ \\
\hline $\mathrm{C}(5)$ & $\mathrm{H}(65)$ & $0.95(4)$ & $\mathrm{C}(8)$ & $\mathrm{H}(66)$ & $1.0(1)$ \\
\hline $\mathrm{C}(8)$ & $\mathrm{H}(67)$ & $1.0(1)$ & $\mathrm{C}(8)$ & $\mathrm{H}(68)$ & $0.95(8)$ \\
\hline $\mathrm{C}(9)$ & $\mathrm{H}(69)$ & $0.95(9)$ & $\mathrm{C}(9)$ & $\mathrm{H}(70)$ & $1.0(1)$ \\
\hline $\mathrm{C}(9)$ & $\mathrm{H}(71)$ & $0.95(6)$ & $\mathrm{C}(10)$ & $\mathrm{H}(72)$ & $0.95(4)$ \\
\hline $\mathrm{C}(10)$ & $\mathrm{H}(73)$ & $1.0(1)$ & $\mathrm{C}(10)$ & $\mathrm{H}(74)$ & $0.9(2)$ \\
\hline $\mathrm{C}(12)$ & $\mathrm{H}(1)$ & $0.95(4)$ & $\mathrm{C}(13)$ & $\mathrm{H}(2)$ & $0.95(5)$ \\
\hline $\mathrm{C}(14)$ & $\mathrm{H}(3)$ & $0.95(5)$ & $\mathrm{C}(15)$ & $\mathrm{H}(4)$ & $0.95(4)$ \\
\hline$C(16)$ & $\mathrm{H}(5)$ & $0.95(3)$ & $\mathrm{C}(18)$ & $\mathrm{H}(6)$ & $0.95(3)$ \\
\hline $\mathrm{C}(19)$ & $\mathrm{H}(7)$ & $0.95(8)$ & $\mathrm{C}(20)$ & $\mathrm{H}(8)$ & $0.95(5)$ \\
\hline $\mathrm{C}(21)$ & $\mathrm{H}(9)$ & $0.95(4)$ & $\mathrm{C}(22)$ & $\mathrm{H}(10)$ & $0.95(4)$ \\
\hline $\mathrm{C}(24)$ & $\mathrm{H}(11)$ & $0.95(3)$ & $\mathrm{C}(25)$ & $\mathrm{H}(12)$ & $0.95(4)$ \\
\hline$C(26)$ & $\mathrm{H}(13)$ & $0.95(3)$ & $\mathrm{C}(27)$ & $\mathrm{H}(14)$ & $0.95(3)$ \\
\hline $\mathrm{C}(28)$ & $\mathrm{H}(15)$ & $0.95(3)$ & $\mathrm{C}(30)$ & $\mathrm{H}(16)$ & $0.95(3)$ \\
\hline $\mathrm{C}(31)$ & $\mathrm{H}(17)$ & $0.95(4)$ & $\mathrm{C}(32)$ & $\mathrm{H}(18)$ & $0.95(7)$ \\
\hline $\mathrm{C}(33)$ & $\mathrm{H}(19)$ & $0.95(3)$ & $\mathrm{C}(34)$ & $\mathrm{H}(20)$ & $0.95(3)$ \\
\hline $\mathrm{C}(35)$ & $\mathrm{H}(45)$ & $0.95(4)$ & $\mathrm{C}(35)$ & $\mathrm{H}(46)$ & $0.95(4)$ \\
\hline$C(36)$ & $\mathrm{H}(78)$ & $0.95(7)$ & $C(36)$ & $\mathrm{H}(79)$ & $0.95(7)$ \\
\hline$C(36)$ & $\mathrm{H}(80)$ & $0.95(4)$ & $C(37)$ & $\mathrm{H}(47)$ & $1.0(1)$ \\
\hline $\mathrm{C}(37)$ & $\mathrm{H}(48)$ & $0.95(9)$ & $\mathrm{C}(38)$ & $\mathrm{H}(81)$ & $0.95(8)$ \\
\hline $\mathrm{C}(38)$ & $\mathrm{H}(82)$ & $0.95(8)$ & $\mathrm{C}(38)$ & $\mathrm{H}(83)$ & $0.95(7)$ \\
\hline $\mathrm{C}(39)$ & $\mathrm{H}(49)$ & $0.95(9)$ & $C(39)$ & $\mathrm{H}(50)$ & $0.95(8)$ \\
\hline $\mathrm{C}(40)$ & $\mathrm{H}(75)$ & $0.95(9)$ & $\mathrm{C}(40)$ & $\mathrm{H}(76)$ & $0.95(6)$ \\
\hline $\mathrm{C}(40)$ & $\mathrm{H}(77)$ & $0.95(6)$ & $\mathrm{C}(41)$ & $\mathrm{H}(43)$ & $0.95(2)$ \\
\hline $\mathrm{C}(41)$ & $\mathrm{H}(44)$ & $0.95(3)$ & $C(43)$ & $\mathrm{H}(84)$ & $0.95(4)$ \\
\hline $\mathrm{C}(43)$ & $\mathrm{H}(85)$ & $0.95(3)$ & $\mathrm{C}(43)$ & $\mathrm{H}(86)$ & $0.95(5)$ \\
\hline $\mathrm{C}(44)$ & $\mathrm{H}(87)$ & $0.95(4)$ & $\mathrm{C}(44)$ & $\mathrm{H}(88)$ & $0.95(3)$ \\
\hline $\mathrm{C}(44)$ & $\mathrm{H}(89)$ & $0.95(6)$ & $C(45)$ & $\mathrm{H}(90)$ & $0.95(5)$ \\
\hline $\mathrm{C}(45)$ & $\mathrm{H}(91)$ & $0.95(3)$ & $C(45)$ & $\mathrm{H}(92)$ & $0.95(4)$ \\
\hline
\end{tabular}




$\begin{array}{llllll}\mathrm{C}(48) & \mathrm{H}(93) & 0.95(7) & \mathrm{C}(48) & \mathrm{H}(94) & 0.95(9) \\ \mathrm{C}(48) & \mathrm{H}(95) & 1.0(1) & \mathrm{C}(49) & \mathrm{H}(96) & 1.0(1) \\ \mathrm{C}(49) & \mathrm{H}(97) & 1.0(1) & \mathrm{C}(49) & \mathrm{H}(98) & 1.0(1) \\ \mathrm{C}(50) & \mathrm{H}(99) & 1.0(1) & \mathrm{C}(50) & \mathrm{H}(100) & 0.95(7) \\ \mathrm{C}(50) & \mathrm{H}(101) & 1.0(1) & \mathrm{C}(52) & \mathrm{H}(21) & 0.95(4) \\ \mathrm{C}(53) & \mathrm{H}(22) & 0.95(6) & \mathrm{C}(54) & \mathrm{H}(23) & 0.95(3) \\ \mathrm{C}(55) & \mathrm{H}(24) & 0.95(4) & \mathrm{C}(56) & \mathrm{H}(25) & 0.95(3) \\ \mathrm{C}(58) & \mathrm{H}(26) & 0.95(3) & \mathrm{C}(59) & \mathrm{H}(27) & 0.95(6) \\ \mathrm{C}(60) & \mathrm{H}(28) & 0.95(7) & \mathrm{C}(61) & \mathrm{H}(29) & 0.95(4) \\ \mathrm{C}(62) & \mathrm{H}(30) & 0.95(3) & \mathrm{C}(64) & \mathrm{H}(31) & 0.95(3) \\ \mathrm{C}(65) & \mathrm{H}(32) & 0.95(3) & \mathrm{C}(66) & \mathrm{H}(33) & 0.95(2) \\ \mathrm{C}(67) & \mathrm{H}(34) & 0.95(4) & \mathrm{C}(68) & \mathrm{H}(35) & 0.95(3) \\ \mathrm{C}(70) & \mathrm{H}(36) & 0.95(4) & \mathrm{C}(71) & \mathrm{H}(37) & 0.95(4) \\ \mathrm{C}(72) & \mathrm{H}(38) & 0.95(6) & \mathrm{C}(73) & \mathrm{H}(39) & 0.95(5) \\ \mathrm{C}(74) & \mathrm{H}(40) & 0.95(3) & \mathrm{C}(75) & \mathrm{H}(55) & 0.95(3) \\ \mathrm{C}(75) & \mathrm{H}(56) & 0.95(5) & \mathrm{C}(76) & \mathrm{H}(102) & 0.95(6) \\ \mathrm{C}(76) & \mathrm{H}(103) & 0.95(5) & \mathrm{C}(76) & \mathrm{H}(104) & 0.95(8) \\ \mathrm{C}(77) & \mathrm{H}(51) & 0.95(6) & \mathrm{C}(77) & \mathrm{H}(52) & 0.95(6) \\ \mathrm{C}(78) & \mathrm{H}(105) & 0.95(7) & \mathrm{C}(78) & \mathrm{H}(106) & 0.95(7) \\ \mathrm{C}(78) & \mathrm{H}(107) & 0.95(8) & \mathrm{C}(79) & \mathrm{H}(53) & 0.95(8) \\ \mathrm{C}(79) & \mathrm{H}(54) & 0.9(1) & \mathrm{C}(80) & \mathrm{H}(108) & 1.0(1) \\ \mathrm{C}(80) & \mathrm{H}(109) & 1.0(1) & \mathrm{C}(80) & \mathrm{H}(110) & 0.95(9)\end{array}$

Table 6. Bond angles (o)

$\begin{array}{llllllll}\text { atom } & \text { atom } & \text { atom } & \text { angle } & \text { atom } & \text { atom } & \text { atom } & \text { angle } \\ \mathrm{P}(1) & \mathrm{Pt}(1) & \mathrm{Si}(1) & 177.4(2) & \mathrm{P}(1) & \mathrm{Pt}(1) & \mathrm{Si}(2) & 96.4(3) \\ \mathrm{P}(1) & \mathrm{Pt}(1) & \mathrm{C}(6) & 92.9(6) & \mathrm{Si}(1) & \mathrm{Pt}(1) & \mathrm{Si}(2) & 81.0(2) \\ \mathrm{Si}(1) & \mathrm{Pt}(1) & \mathrm{C}(6) & 89.7(6) & \mathrm{Si}(2) & \mathrm{Pt}(1) & \mathrm{C}(6) & 170.3(6) \\ \mathrm{P}(2) & \mathrm{Pt}(2) & \mathrm{Si}(3) & 176.5(3) & \mathrm{P}(2) & \mathrm{Pt}(2) & \mathrm{Si}(4) & 96.8(2) \\ \mathrm{P}(2) & \mathrm{Pt}(2) & \mathrm{C}(46) & 94.7(5) & \mathrm{Si}(3) & \mathrm{Pt}(2) & \mathrm{Si}(4) & 79.8(2) \\ \mathrm{Si}(3) & \mathrm{Pt}(2) & \mathrm{C}(46) & 88.8(5) & \mathrm{Si}(4) & \mathrm{Pt}(2) & \mathrm{C}(46) & 168.4(5) \\ \mathrm{C}(35) & \mathrm{P}(1) & \mathrm{C}(37) & 76(2) & \mathrm{C}(35) & \mathrm{P}(1) & \mathrm{C}(39) & 100(2) \\ \mathrm{C}(35) & \mathrm{P}(1) & \mathrm{Pt}(1) & 121(1) & \mathrm{C}(37) & \mathrm{P}(1) & \mathrm{C}(39) & 85(3) \\ \mathrm{C}(37) & \mathrm{P}(1) & \mathrm{Pt}(1) & 112(2) & \mathrm{C}(39) & \mathrm{P}(1) & \mathrm{Pt}(1) & 136(1) \\ \mathrm{C}(75) & \mathrm{P}(2) & \mathrm{C}(77) & 100(1) & \mathrm{C}(75) & \mathrm{P}(2) & \mathrm{C}(79) & 94(2) \\ \mathrm{C}(75) & \mathrm{P}(2) & \mathrm{Pt}(2) & 121.2(8) & \mathrm{C}(77) & \mathrm{P}(2) & \mathrm{C}(79) & 83(2) \\ \mathrm{C}(77) & \mathrm{P}(2) & \mathrm{Pt}(2) & 135(1) & \mathrm{C}(79) & \mathrm{P}(2) & \mathrm{Pt}(2) & 105(1) \\ \mathrm{N}(1) & \mathrm{Si}(1) & \mathrm{C}(11) & 109.6(6) & \mathrm{N}(1) & \mathrm{Si}(1) & \mathrm{C}(17) & 111.8(8) \\ \mathrm{N}(1) & \mathrm{Si}(1) & \mathrm{Pt}(1) & 113.0(6) & \mathrm{C}(11) & \mathrm{Si}(1) & \mathrm{C}(17) & 107(1) \\ \mathrm{C}(11) & \mathrm{Si}(1) & \mathrm{Pt}(1) & 108.7(6) & \mathrm{C}(17) & \mathrm{Si}(1) & \mathrm{Pt}(1) & 106.4(9) \\ \mathrm{C}(1) & \mathrm{Si}(2) & \mathrm{C}(23) & 103.9(7) & \mathrm{C}(1) & \mathrm{Si}(2) & \mathrm{C}(29) & 105.6(6) \\ \mathrm{C}(1) & \mathrm{Si}(2) & \mathrm{Pt}(1) & 106.8(6) & \mathrm{C}(23) & \mathrm{Si}(2) & \mathrm{C}(29) & 108.2(8) \\ \mathrm{C}(23) & \mathrm{Si}(2) & \mathrm{Pt}(1) & 116.7(4) & \mathrm{C}(29) & \mathrm{Si}(2) & \mathrm{Pt}(1) & 114.5(4) \\ \mathrm{N}(3) & \mathrm{Si}(3) & \mathrm{C}(51) & 109.8(8) & \mathrm{N}(3) & \mathrm{Si}(3) & \mathrm{C}(57) & 111(1)\end{array}$




\begin{tabular}{|c|c|c|c|c|c|c|c|}
\hline $\mathrm{N}(3)$ & $\operatorname{Si}(3)$ & $\operatorname{Pt}(2)$ & $113.6(5)$ & $\mathrm{C}(51)$ & $\operatorname{Si}(3)$ & $C(57)$ & 108.1(9) \\
\hline $\mathrm{C}(51)$ & $\operatorname{Si}(3)$ & $\operatorname{Pt}(2)$ & $108.5(5)$ & $C(57)$ & $\operatorname{Si}(3)$ & $\operatorname{Pt}(2)$ & $105.5(7)$ \\
\hline $\mathrm{C}(41)$ & $\operatorname{Si}(4)$ & $C(63)$ & $101.8(6)$ & $\mathrm{C}(41)$ & $\operatorname{Si}(4)$ & $\mathrm{C}(69)$ & $104.5(7)$ \\
\hline $\mathrm{C}(41)$ & $\operatorname{Si}(4)$ & $\operatorname{Pt}(2)$ & $108.7(6)$ & $\mathrm{C}(63)$ & $\operatorname{Si}(4)$ & $\mathrm{C}(69)$ & $108.9(7)$ \\
\hline C(63) & $\operatorname{Si}(4)$ & $\operatorname{Pt}(2)$ & $116.3(6)$ & $\mathrm{C}(69)$ & $\operatorname{Si}(4)$ & $\operatorname{Pt}(2)$ & $115.1(5)$ \\
\hline $\mathrm{C}(1)$ & $\mathrm{N}(1)$ & $C(2)$ & 114(1) & $\mathrm{C}(1)$ & $\mathrm{N}(1)$ & $\operatorname{Si}(1)$ & $113.4(9)$ \\
\hline$C(2)$ & $\mathrm{N}(1)$ & $\operatorname{Si}(1)$ & $125(1)$ & $C(6)$ & $\mathrm{N}(2)$ & $\mathrm{C}(7)$ & $170(1)$ \\
\hline $\mathrm{C}(41)$ & $\mathrm{N}(3)$ & $\mathrm{C}(42)$ & 112(1) & $\mathrm{C}(41)$ & $\mathrm{N}(3)$ & $\operatorname{Si}(3)$ & 114(1) \\
\hline$C(42)$ & $\mathrm{N}(3)$ & $\operatorname{Si}(3)$ & $126(1)$ & $C(46)$ & $\mathrm{N}(4)$ & $\mathrm{C}(47)$ & 171(1) \\
\hline $\operatorname{Si}(2)$ & $\mathrm{C}(1)$ & $\mathrm{N}(1)$ & $110(1)$ & $\mathrm{C}(3)$ & $\mathrm{C}(2)$ & $\mathrm{C}(4)$ & 103(1) \\
\hline$C(3)$ & $\mathrm{C}(2)$ & $C(5)$ & $110(2)$ & $\mathrm{C}(3)$ & $\mathrm{C}(2)$ & $\mathrm{N}(1)$ & 113(1) \\
\hline$C(4)$ & $\mathrm{C}(2)$ & $C(5)$ & 107(1) & $\mathrm{C}(4)$ & $\mathrm{C}(2)$ & $\mathrm{N}(1)$ & $107(2)$ \\
\hline$C(5)$ & $\mathrm{C}(2)$ & $\mathrm{N}(1)$ & $114(1)$ & $\operatorname{Pt}(1)$ & $\mathrm{C}(6)$ & $\mathrm{N}(2)$ & $177(1)$ \\
\hline $\mathrm{C}(8)$ & $\mathrm{C}(7)$ & $\mathrm{C}(9)$ & $107(5)$ & $\mathrm{C}(8)$ & $\mathrm{C}(7)$ & $\mathrm{C}(10)$ & $117(4)$ \\
\hline $\mathrm{C}(8)$ & $\mathrm{C}(7)$ & $\mathrm{N}(2)$ & $103(2)$ & $\mathrm{C}(9)$ & $\mathrm{C}(7)$ & $\mathrm{C}(10)$ & $108(5)$ \\
\hline $\mathrm{C}(9)$ & $\mathrm{C}(7)$ & $\mathrm{N}(2)$ & $108(1)$ & $\mathrm{C}(10)$ & $\mathrm{C}(7)$ & $\mathrm{N}(2)$ & 111(3) \\
\hline$C(12)$ & $\mathrm{C}(11)$ & $C(16)$ & $114(1)$ & $\mathrm{C}(12)$ & $\mathrm{C}(11)$ & $\operatorname{Si}(1)$ & 121(1) \\
\hline$C(16)$ & $\mathrm{C}(11)$ & $\operatorname{Si}(1)$ & $123(1)$ & $\mathrm{C}(13)$ & $\mathrm{C}(12)$ & $\mathrm{C}(11)$ & $124(2)$ \\
\hline $\mathrm{C}(14)$ & $\mathrm{C}(13)$ & $C(12)$ & $114(2)$ & $C(15)$ & $\mathrm{C}(14)$ & $\mathrm{C}(13)$ & $125(2)$ \\
\hline$C(16)$ & $\mathrm{C}(15)$ & $C(14)$ & $116(2)$ & $\mathrm{C}(11)$ & $C(16)$ & $\mathrm{C}(15)$ & $124(1)$ \\
\hline $\mathrm{C}(18)$ & $\mathrm{C}(17)$ & $\mathrm{C}(22)$ & $114(2)$ & $\mathrm{C}(18)$ & $\mathrm{C}(17)$ & $\mathrm{Si}(1)$ & $126(1)$ \\
\hline $\mathrm{C}(22)$ & $\mathrm{C}(17)$ & $\operatorname{Si}(1)$ & $118(2)$ & $C(19)$ & $\mathrm{C}(18)$ & $\mathrm{C}(17)$ & $116(2)$ \\
\hline $\mathrm{C}(20)$ & $\mathrm{C}(19)$ & $\mathrm{C}(18)$ & $125(4)$ & $\mathrm{C}(21)$ & $\mathrm{C}(20)$ & $\mathrm{C}(19)$ & $118(3)$ \\
\hline$C(22)$ & $\mathrm{C}(21)$ & $C(20)$ & $123(2)$ & $C(17)$ & $\mathrm{C}(22)$ & $\mathrm{C}(21)$ & $120(2)$ \\
\hline $\mathrm{C}(24)$ & $\mathrm{C}(23)$ & $\mathrm{C}(28)$ & $114(1)$ & $\mathrm{C}(24)$ & $\mathrm{C}(23)$ & $\operatorname{Si}(2)$ & $128(1)$ \\
\hline $\mathrm{C}(28)$ & $\mathrm{C}(23)$ & $\operatorname{Si}(2)$ & 117(1) & $C(25)$ & $\mathrm{C}(24)$ & $\mathrm{C}(23)$ & $122(1)$ \\
\hline $\mathrm{C}(26)$ & $\mathrm{C}(25)$ & $\mathrm{C}(24)$ & $122(2)$ & $\mathrm{C}(27)$ & $C(26)$ & $\mathrm{C}(25)$ & 119(1) \\
\hline $\mathrm{C}(28)$ & $\mathrm{C}(27)$ & $C(26)$ & $120(1)$ & $\mathrm{C}(23)$ & $\mathrm{C}(28)$ & $\mathrm{C}(27)$ & $121(2)$ \\
\hline$C(30)$ & $\mathrm{C}(29)$ & $C(34)$ & 117(1) & $\mathrm{C}(30)$ & $\mathrm{C}(29)$ & $\operatorname{Si}(2)$ & 118(1) \\
\hline$C(34)$ & $\mathrm{C}(29)$ & $\operatorname{Si}(2)$ & $124(1)$ & $\mathrm{C}(31)$ & $\mathrm{C}(30)$ & $\mathrm{C}(29)$ & 119(1) \\
\hline$C(32)$ & $\mathrm{C}(31)$ & $C(30)$ & $119(2)$ & $C(33)$ & $\mathrm{C}(32)$ & $\mathrm{C}(31)$ & 121(3) \\
\hline$C(34)$ & $\mathrm{C}(33)$ & $C(32)$ & $116(2)$ & $C(29)$ & $\mathrm{C}(34)$ & $\mathrm{C}(33)$ & $125(1)$ \\
\hline$C(36)$ & $\mathrm{C}(35)$ & $\mathrm{P}(1)$ & $108(1)$ & $\mathrm{C}(38)$ & $\mathrm{C}(37)$ & $\mathrm{P}(1)$ & $130(5)$ \\
\hline$C(40)$ & $\mathrm{C}(39)$ & $\mathrm{P}(1)$ & 101(4) & $\operatorname{Si}(4)$ & $\mathrm{C}(41)$ & $\mathrm{N}(3)$ & 108(1) \\
\hline$C(43)$ & $C(42)$ & $C(44)$ & $113(2)$ & $\mathrm{C}(43)$ & $\mathrm{C}(42)$ & $\mathrm{C}(45)$ & 107(1) \\
\hline$C(43)$ & $\mathrm{C}(42)$ & $\mathrm{N}(3)$ & $110(1)$ & $\mathrm{C}(44)$ & $\mathrm{C}(42)$ & $\mathrm{C}(45)$ & $105(2)$ \\
\hline$C(44)$ & $\mathrm{C}(42)$ & $\mathrm{N}(3)$ & 112(1) & $C(45)$ & $\mathrm{C}(42)$ & $\mathrm{N}(3)$ & 107(1) \\
\hline $\operatorname{Pt}(2)$ & $C(46)$ & $\mathrm{N}(4)$ & 177(1) & $\mathrm{C}(48)$ & $\mathrm{C}(47)$ & $\mathrm{C}(49)$ & $109(4)$ \\
\hline$C(48)$ & $\mathrm{C}(47)$ & $C(50)$ & $105(4)$ & $\mathrm{C}(48)$ & $\mathrm{C}(47)$ & $\mathrm{N}(4)$ & $110(3)$ \\
\hline C(49) & $\mathrm{C}(47)$ & $C(50)$ & $117(4)$ & $C(49)$ & $\mathrm{C}(47)$ & $\mathrm{N}(4)$ & 103(4) \\
\hline $\mathrm{C}(50)$ & $\mathrm{C}(47)$ & $\mathrm{N}(4)$ & 108(3) & $C(52)$ & $\mathrm{C}(51)$ & $C(56)$ & $116(1)$ \\
\hline$C(52)$ & $C(51)$ & $\operatorname{Si}(3)$ & $120(1)$ & $C(56)$ & $\mathrm{C}(51)$ & $\mathrm{Si}(3)$ & $122(1)$ \\
\hline$C(53)$ & $C(52)$ & $\mathrm{C}(51)$ & $121(2)$ & $C(54)$ & $C(53)$ & $\mathrm{C}(52)$ & $117(3)$ \\
\hline$C(55)$ & $C(54)$ & $C(53)$ & $123(2)$ & $C(56)$ & $C(55)$ & $\mathrm{C}(54)$ & $116(2)$ \\
\hline$C(51)$ & $C(56)$ & $C(55)$ & $124(2)$ & $\mathrm{C}(58)$ & $C(57)$ & $\mathrm{C}(62)$ & $112(2)$ \\
\hline
\end{tabular}




$\begin{array}{llllllll}\mathrm{C}(58) & \mathrm{C}(57) & \mathrm{Si}(3) & 126(1) & \mathrm{C}(62) & \mathrm{C}(57) & \mathrm{Si}(3) & 120(1) \\ \mathrm{C}(59) & \mathrm{C}(58) & \mathrm{C}(57) & 126(2) & \mathrm{C}(60) & \mathrm{C}(59) & \mathrm{C}(58) & 116(3) \\ \mathrm{C}(61) & \mathrm{C}(60) & \mathrm{C}(59) & 122(4) & \mathrm{C}(62) & \mathrm{C}(61) & \mathrm{C}(60) & 119(2) \\ \mathrm{C}(57) & \mathrm{C}(62) & \mathrm{C}(61) & 122(2) & \mathrm{C}(64) & \mathrm{C}(63) & \mathrm{C}(68) & 115(1) \\ \mathrm{C}(64) & \mathrm{C}(63) & \mathrm{Si}(4) & 126(1) & \mathrm{C}(68) & \mathrm{C}(63) & \mathrm{Si}(4) & 118(1) \\ \mathrm{C}(65) & \mathrm{C}(64) & \mathrm{C}(63) & 122(2) & \mathrm{C}(66) & \mathrm{C}(65) & \mathrm{C}(64) & 119(2) \\ \mathrm{C}(67) & \mathrm{C}(66) & \mathrm{C}(65) & 120(1) & \mathrm{C}(68) & \mathrm{C}(67) & \mathrm{C}(66) & 119(2) \\ \mathrm{C}(63) & \mathrm{C}(68) & \mathrm{C}(67) & 122(2) & \mathrm{C}(70) & \mathrm{C}(69) & \mathrm{C}(74) & 118(2) \\ \mathrm{C}(70) & \mathrm{C}(69) & \mathrm{Si}(4) & 118(1) & \mathrm{C}(74) & \mathrm{C}(69) & \mathrm{Si}(4) & 123(1) \\ \mathrm{C}(71) & \mathrm{C}(70) & \mathrm{C}(69) & 118(2) & \mathrm{C}(72) & \mathrm{C}(71) & \mathrm{C}(70) & 121(2) \\ \mathrm{C}(73) & \mathrm{C}(72) & \mathrm{C}(71) & 120(3) & \mathrm{C}(74) & \mathrm{C}(73) & \mathrm{C}(72) & 119(3) \\ \mathrm{C}(69) & \mathrm{C}(74) & \mathrm{C}(73) & 122(2) & \mathrm{C}(76) & \mathrm{C}(75) & \mathrm{P}(2) & 107(2) \\ \mathrm{C}(78) & \mathrm{C}(77) & \mathrm{P}(2) & 107(2) & \mathrm{C}(80) & \mathrm{C}(79) & \mathrm{P}(2) & 117(4)\end{array}$

Table 7. Bond angles involving hydrogens ( $\left(^{*}\right)$

$\begin{array}{llllllll}\text { atom } & \text { atom } & \text { atom } & \text { angle } & \text { atom } & \text { atom } & \text { atom } & \text { angle } \\ \mathrm{H}(41) & \mathrm{C}(1) & \mathrm{H}(42) & 109(1) & \mathrm{H}(41) & \mathrm{C}(1) & \mathrm{Si}(2) & 109(2) \\ \mathrm{H}(41) & \mathrm{C}(1) & \mathrm{N}(1) & 108(1) & \mathrm{H}(42) & \mathrm{C}(1) & \mathrm{Si}(2) & 109(1) \\ \mathrm{H}(42) & \mathrm{C}(1) & \mathrm{N}(1) & 109(2) & \mathrm{H}(57) & \mathrm{C}(3) & \mathrm{H}(58) & 109(5) \\ \mathrm{H}(57) & \mathrm{C}(3) & \mathrm{H}(59) & 109(3) & \mathrm{H}(57) & \mathrm{C}(3) & \mathrm{C}(2) & 107(2) \\ \mathrm{H}(58) & \mathrm{C}(3) & \mathrm{H}(59) & 109(3) & \mathrm{H}(58) & \mathrm{C}(3) & \mathrm{C}(2) & 108(2) \\ \mathrm{H}(59) & \mathrm{C}(3) & \mathrm{C}(2) & 112(4) & \mathrm{H}(60) & \mathrm{C}(4) & \mathrm{H}(61) & 109(3) \\ \mathrm{H}(60) & \mathrm{C}(4) & \mathrm{H}(62) & 109(4) & \mathrm{H}(60) & \mathrm{C}(4) & \mathrm{C}(2) & 110(2) \\ \mathrm{H}(61) & \mathrm{C}(4) & \mathrm{H}(62) & 109(3) & \mathrm{H}(61) & \mathrm{C}(4) & \mathrm{C}(2) & 108(3) \\ \mathrm{H}(62) & \mathrm{C}(4) & \mathrm{C}(2) & 109(2) & \mathrm{H}(63) & \mathrm{C}(5) & \mathrm{H}(64) & 109(3) \\ \mathrm{H}(63) & \mathrm{C}(5) & \mathrm{H}(65) & 109(3) & \mathrm{H}(63) & \mathrm{C}(5) & \mathrm{C}(2) & 111(3) \\ \mathrm{H}(64) & \mathrm{C}(5) & \mathrm{H}(65) & 109(4) & \mathrm{H}(64) & \mathrm{C}(5) & \mathrm{C}(2) & 109(2) \\ \mathrm{H}(65) & \mathrm{C}(5) & \mathrm{C}(2) & 107(2) & \mathrm{H}(66) & \mathrm{C}(8) & \mathrm{H}(67) & 109(8) \\ \mathrm{H}(66) & \mathrm{C}(8) & \mathrm{H}(68) & 109(8) & \mathrm{H}(66) & \mathrm{C}(8) & \mathrm{C}(7) & 125(10) \\ \mathrm{H}(67) & \mathrm{C}(8) & \mathrm{H}(68) & 109(11) & \mathrm{H}(67) & \mathrm{C}(8) & \mathrm{C}(7) & 99(7) \\ \mathrm{H}(68) & \mathrm{C}(8) & \mathrm{C}(7) & 102(7) & \mathrm{H}(69) & \mathrm{C}(9) & \mathrm{H}(70) & 109(5) \\ \mathrm{H}(69) & \mathrm{C}(9) & \mathrm{H}(71) & 109(10) & \mathrm{H}(69) & \mathrm{C}(9) & \mathrm{C}(7) & 112(4) \\ \mathrm{H}(70) & \mathrm{C}(9) & \mathrm{H}(71) & 109(6) & \mathrm{H}(70) & \mathrm{C}(9) & \mathrm{C}(7) & 105(8) \\ \mathrm{H}(71) & \mathrm{C}(9) & \mathrm{C}(7) & 111(4) & \mathrm{H}(72) & \mathrm{C}(10) & \mathrm{H}(73) & 109(8) \\ \mathrm{H}(72) & \mathrm{C}(10) & \mathrm{H}(74) & 109(11) & \mathrm{H}(72) & \mathrm{C}(10) & \mathrm{C}(7) & 110(7) \\ \mathrm{H}(73) & \mathrm{C}(10) & \mathrm{H}(74) & 109(10) & \mathrm{H}(73) & \mathrm{C}(10) & \mathrm{C}(7) & 113(8) \\ \mathrm{H}(74) & \mathrm{C}(10) & \mathrm{C}(7) & 103(5) & \mathrm{C}(13) & \mathrm{C}(12) & \mathrm{H}(1) & 119(2) \\ \mathrm{H}(1) & \mathrm{C}(12) & \mathrm{C}(11) & 116(2) & \mathrm{C}(14) & \mathrm{C}(13) & \mathrm{H}(2) & 123(3) \\ \mathrm{H}(2) & \mathrm{C}(13) & \mathrm{C}(12) & 121(4) & \mathrm{C}(15) & \mathrm{C}(14) & \mathrm{H}(3) & 117(6) \\ \mathrm{H}(3) & \mathrm{C}(14) & \mathrm{C}(13) & 116(4) & \mathrm{C}(16) & \mathrm{C}(15) & \mathrm{H}(4) & 122(2) \\ \mathrm{H}(4) & \mathrm{C}(15) & \mathrm{C}(14) & 120(3) & \mathrm{H}(5) & \mathrm{C}(16) & \mathrm{C}(11) & 117(2) \\ \mathrm{H}(5) & \mathrm{C}(16) & \mathrm{C}(15) & 118(2) & \mathrm{C}(19) & \mathrm{C}(18) & \mathrm{H}(6) & 123(4) \\ \mathrm{H}(6) & \mathrm{C}(18) & \mathrm{C}(17) & 119(3) & \mathrm{C}(20) & \mathrm{C}(19) & \mathrm{H}(7) & 117(4) \\ \mathrm{H}(7) & \mathrm{C}(19) & \mathrm{C}(18) & 117(4) & \mathrm{C}(21) & \mathrm{C}(20) & \mathrm{H}(8) & 120(5)\end{array}$




\begin{tabular}{|c|c|c|c|c|c|c|c|}
\hline $\mathrm{H}(8)$ & $\mathrm{C}(20)$ & $\mathrm{C}(19)$ & $121(6)$ & $\mathrm{C}(22)$ & $\mathrm{C}(21)$ & $\mathrm{H}(9)$ & $118(4)$ \\
\hline $\mathrm{H}(9)$ & $\mathrm{C}(21)$ & $\mathrm{C}(20)$ & $118(3)$ & $\mathrm{H}(10)$ & $\mathrm{C}(22)$ & $\mathrm{C}(17)$ & $115(2)$ \\
\hline $\mathrm{H}(10)$ & $\mathrm{C}(22)$ & $\mathrm{C}(21)$ & $123(2)$ & $\mathrm{C}(25)$ & $C(24)$ & $\mathrm{H}(11)$ & $118(2)$ \\
\hline $\mathrm{H}(11)$ & $C(24)$ & $\mathrm{C}(23)$ & $119(2)$ & $\mathrm{C}(26)$ & $C(25)$ & $\mathrm{H}(12)$ & $119(2)$ \\
\hline $\mathrm{H}(12)$ & $\mathrm{C}(25)$ & $\mathrm{C}(24)$ & $118(2)$ & $\mathrm{C}(27)$ & $C(26)$ & $\mathrm{H}(13)$ & $120(2)$ \\
\hline $\mathrm{H}(13)$ & $C(26)$ & $\mathrm{C}(25)$ & $119(3)$ & $\mathrm{C}(28)$ & $\mathrm{C}(27)$ & $\mathrm{H}(14)$ & $119(3)$ \\
\hline $\mathrm{H}(14)$ & $\mathrm{C}(27)$ & $C(26)$ & $120(2)$ & $\mathrm{H}(15)$ & $\mathrm{C}(28)$ & $C(23)$ & $119(2)$ \\
\hline$H(15)$ & $\mathrm{C}(28)$ & $\mathrm{C}(27)$ & $119(2)$ & $\mathrm{C}(31)$ & $\mathrm{C}(30)$ & $\mathrm{H}(16)$ & $119(2)$ \\
\hline $\mathrm{H}(16)$ & $C(30)$ & $C(29)$ & $120(2)$ & $\mathrm{C}(32)$ & $\mathrm{C}(31)$ & $\mathrm{H}(17)$ & $118(3)$ \\
\hline $\mathrm{H}(17)$ & $\mathrm{C}(31)$ & $C(30)$ & $122(2)$ & C(33) & $\mathrm{C}(32)$ & $\mathrm{H}(18)$ & $121(4)$ \\
\hline $\mathrm{H}(18)$ & $C(32)$ & $\mathrm{C}(31)$ & $115(4)$ & $C(34)$ & $\mathrm{C}(33)$ & $\mathrm{H}(19)$ & $123(3)$ \\
\hline $\mathrm{H}(19)$ & $\mathrm{C}(33)$ & $C(32)$ & $119(3)$ & $\mathrm{H}(20)$ & $\mathrm{C}(34)$ & $C(29)$ & $117(2)$ \\
\hline $\mathrm{H}(20)$ & $\mathrm{C}(34)$ & $\mathrm{C}(33)$ & $117(2)$ & $C(36)$ & $\mathrm{C}(35)$ & $\mathrm{H}(45)$ & $107(3)$ \\
\hline$C(36)$ & $C(35)$ & $\mathrm{H}(46)$ & 111(3) & $\mathrm{H}(45)$ & $\mathrm{C}(35)$ & $\mathrm{H}(46)$ & $109(2)$ \\
\hline $\mathrm{H}(45)$ & $\mathrm{C}(35)$ & $\mathrm{P}(1)$ & $107(2)$ & $\mathrm{H}(46)$ & $\mathrm{C}(35)$ & $\mathrm{P}(1)$ & $111(2)$ \\
\hline $\mathrm{H}(78)$ & $C(36)$ & $\mathrm{H}(79)$ & $109(4)$ & $\mathrm{H}(78)$ & $C(36)$ & $\mathrm{H}(80)$ & $109(5)$ \\
\hline $\mathrm{H}(78)$ & $C(36)$ & $C(35)$ & $105(4)$ & $\mathrm{H}(79)$ & $C(36)$ & $\mathrm{H}(80)$ & $109(6)$ \\
\hline $\mathrm{H}(79)$ & $C(36)$ & $C(35)$ & $109(4)$ & $\mathrm{H}(80)$ & C(36) & $C(35)$ & $113(3)$ \\
\hline $\mathrm{C}(38)$ & $\mathrm{C}(37)$ & $\mathrm{H}(47)$ & $99(7)$ & $\mathrm{C}(38)$ & $\mathrm{C}(37)$ & $\mathrm{H}(48)$ & $110(7)$ \\
\hline $\mathrm{H}(47)$ & $\mathrm{C}(37)$ & $\mathrm{H}(48)$ & $109(10)$ & $\mathrm{H}(47)$ & $\mathrm{C}(37)$ & $\mathrm{P}(1)$ & $107(6)$ \\
\hline $\mathrm{H}(48)$ & $\mathrm{C}(37)$ & $\mathrm{P}(1)$ & $98(6)$ & $\mathrm{H}(81)$ & $\mathrm{C}(38)$ & $\mathrm{H}(82)$ & $109(8)$ \\
\hline $\mathrm{H}(81)$ & $\mathrm{C}(38)$ & $\mathrm{H}(83)$ & $109(5)$ & $\mathrm{H}(81)$ & $\mathrm{C}(38)$ & $C(37)$ & $102(6)$ \\
\hline $\mathrm{H}(82)$ & $\mathrm{C}(38)$ & $\mathrm{H}(83)$ & 109(7) & $\mathrm{H}(82)$ & $\mathrm{C}(38)$ & $C(37)$ & $114(5)$ \\
\hline $\mathrm{H}(83)$ & $\mathrm{C}(38)$ & $\mathrm{C}(37)$ & 111(8) & $\mathrm{C}(40)$ & C(39) & $\mathrm{H}(49)$ & $110(8)$ \\
\hline$C(40)$ & C(39) & $\mathrm{H}(50)$ & $108(6)$ & H(49) & C(39) & $\mathrm{H}(50)$ & $109(8)$ \\
\hline $\mathrm{H}(49)$ & C(39) & $\mathrm{P}(1)$ & $115(7)$ & $\mathrm{H}(50)$ & C(39) & $\mathrm{P}(1)$ & $110(8)$ \\
\hline $\mathrm{H}(75)$ & $\mathrm{C}(40)$ & $\mathrm{H}(76)$ & $109(5)$ & $\mathrm{H}(75)$ & $\mathrm{C}(40)$ & $\mathrm{H}(77)$ & $109(7)$ \\
\hline $\mathrm{H}(75)$ & $\mathrm{C}(40)$ & $C(39)$ & $113(5)$ & $\mathrm{H}(76)$ & $\mathrm{C}(40)$ & $\mathrm{H}(77)$ & $109(6)$ \\
\hline $\mathrm{H}(76)$ & $\mathrm{C}(40)$ & $\mathrm{C}(39)$ & $108(6)$ & $\mathrm{H}(77)$ & $\mathrm{C}(40)$ & C(39) & $106(4)$ \\
\hline $\mathrm{H}(43)$ & $\mathrm{C}(41)$ & $\mathrm{H}(44)$ & $109(2)$ & $\mathrm{H}(43)$ & $\mathrm{C}(41)$ & $\mathrm{Si}(4)$ & 109(1) \\
\hline $\mathrm{H}(43)$ & $\mathrm{C}(41)$ & $\mathrm{N}(3)$ & $108(1)$ & $\mathrm{H}(44)$ & $\mathrm{C}(41)$ & $\mathrm{Si}(4)$ & $110(1)$ \\
\hline $\mathrm{H}(44)$ & $\mathrm{C}(41)$ & $\mathrm{N}(3)$ & 111(1) & $\mathrm{H}(84)$ & $\mathrm{C}(43)$ & $\mathrm{H}(85)$ & $109(3)$ \\
\hline $\mathrm{H}(84)$ & $C(43)$ & $\mathrm{H}(86)$ & 109(3) & $\mathrm{H}(84)$ & $C(43)$ & $C(42)$ & $109(2)$ \\
\hline $\mathrm{H}(85)$ & $C(43)$ & $\mathrm{H}(86)$ & $109(3)$ & $\mathrm{H}(85)$ & $C(43)$ & $C(42)$ & $108(2)$ \\
\hline $\mathrm{H}(86)$ & $C(43)$ & $C(42)$ & $109(2)$ & $\mathrm{H}(87)$ & $C(44)$ & $\mathrm{H}(88)$ & $109(3)$ \\
\hline $\mathrm{H}(87)$ & $C(44)$ & $\mathrm{H}(89)$ & $109(3)$ & $\mathrm{H}(87)$ & $C(44)$ & $C(42)$ & $110(3)$ \\
\hline $\mathrm{H}(88)$ & $C(44)$ & $\mathrm{H}(89)$ & $109(4)$ & $\mathrm{H}(88)$ & $C(44)$ & $C(42)$ & $108(2)$ \\
\hline $\mathrm{H}(89)$ & $C(44)$ & $C(42)$ & $109(2)$ & $\mathrm{H}(90)$ & $C(45)$ & $\mathrm{H}(91)$ & $109(3)$ \\
\hline $\mathrm{H}(90)$ & $C(45)$ & $\mathrm{H}(92)$ & $109(4)$ & $\mathrm{H}(90)$ & $C(45)$ & $C(42)$ & $108(3)$ \\
\hline $\mathrm{H}(91)$ & $C(45)$ & $\mathrm{H}(92)$ & 109(3) & $\mathrm{H}(91)$ & $C(45)$ & $\mathrm{C}(42)$ & $110(3)$ \\
\hline $\mathrm{H}(92)$ & $C(45)$ & $\mathrm{C}(42)$ & $109(2)$ & H(93) & $\mathrm{C}(48)$ & $\mathrm{H}(94)$ & $109(7)$ \\
\hline $\mathrm{H}(93)$ & $\mathrm{C}(48)$ & $\mathrm{H}(95)$ & 109(10) & $\mathrm{H}(93)$ & $\mathrm{C}(48)$ & $C(47)$ & $105(7)$ \\
\hline $\mathrm{H}(94)$ & $C(48)$ & $\mathrm{H}(95)$ & 109(8) & $\mathrm{H}(94)$ & $\mathrm{C}(48)$ & $C(47)$ & $110(8)$ \\
\hline $\mathrm{H}(95)$ & $\mathrm{C}(48)$ & $\mathrm{C}(47)$ & $112(6)$ & $\mathrm{H}(96)$ & C(49) & $\mathrm{H}(97)$ & $109(10)$ \\
\hline $\mathrm{H}(96)$ & C(49) & $\mathrm{H}(98)$ & $109(10)$ & $\mathrm{H}(96)$ & $C(49)$ & $C(47)$ & $106(8)$ \\
\hline
\end{tabular}




\begin{tabular}{|c|c|c|c|c|c|c|c|}
\hline $\mathrm{H}(97)$ & $C(49)$ & $\mathrm{H}(98)$ & 109(11) & $\mathrm{H}(97)$ & $\mathrm{C}(49)$ & $C(47)$ & $102(6)$ \\
\hline $\mathrm{H}(98)$ & $\mathrm{C}(49)$ & $C(47)$ & $119(7)$ & $\mathrm{H}(99)$ & $C(50)$ & $\mathrm{H}(100)$ & $109(7)$ \\
\hline $\mathrm{H}(99)$ & $C(50)$ & $\mathrm{H}(101)$ & $109(8)$ & $\mathrm{H}(99)$ & $\mathrm{C}(50)$ & $C(47)$ & $112(7)$ \\
\hline $\mathrm{H}(100)$ & $C(50)$ & $\mathrm{H}(101)$ & 109(9) & $\mathrm{H}(100)$ & $\mathrm{C}(50)$ & $C(47)$ & $104(5)$ \\
\hline $\mathrm{H}(101)$ & $C(50)$ & $\mathrm{C}(47)$ & $111(5)$ & $\mathrm{C}(53)$ & $\mathrm{C}(52)$ & $\mathrm{H}(21)$ & $123(3)$ \\
\hline $\mathrm{H}(21)$ & $C(52)$ & $C(51)$ & $114(2)$ & $C(54)$ & $\mathrm{C}(53)$ & $\mathrm{H}(22)$ & $123(3)$ \\
\hline $\mathrm{H}(22)$ & $C(53)$ & $C(52)$ & $119(4)$ & $\mathrm{C}(55)$ & $\mathrm{C}(54)$ & $\mathrm{H}(23)$ & $117(4)$ \\
\hline $\mathrm{H}(23)$ & $C(54)$ & $C(53)$ & $119(5)$ & $C(56)$ & $C(55)$ & $\mathrm{H}(24)$ & $120(3)$ \\
\hline $\mathrm{H}(24)$ & $C(55)$ & $C(54)$ & $122(2)$ & $\mathrm{H}(25)$ & $C(56)$ & $\mathrm{C}(51)$ & $118(1)$ \\
\hline $\mathrm{H}(25)$ & $C(56)$ & $C(55)$ & $117(2)$ & $\mathrm{C}(59)$ & $\mathrm{C}(58)$ & $\mathrm{H}(26)$ & $120(3)$ \\
\hline $\mathrm{H}(26)$ & $\mathrm{C}(58)$ & $C(57)$ & $112(3)$ & $\mathrm{C}(60)$ & $\mathrm{C}(59)$ & $\mathrm{H}(27)$ & $123(6)$ \\
\hline $\mathrm{H}(27)$ & C(59) & $\mathrm{C}(58)$ & $120(5)$ & $\mathrm{C}(61)$ & $\mathrm{C}(60)$ & $\mathrm{H}(28)$ & 119(4) \\
\hline $\mathrm{H}(28)$ & $C(60)$ & $C(59)$ & $117(4)$ & $C(62)$ & $\mathrm{C}(61)$ & $\mathrm{H}(29)$ & 121(4) \\
\hline $\mathrm{H}(29)$ & $\mathrm{C}(61)$ & $C(60)$ & $118(5)$ & $\mathrm{H}(30)$ & $\mathrm{C}(62)$ & $C(57)$ & $115(3)$ \\
\hline $\mathrm{H}(30)$ & $C(62)$ & $\mathrm{C}(61)$ & $122(2)$ & $C(65)$ & $\mathrm{C}(64)$ & $\mathrm{H}(31)$ & $118(2)$ \\
\hline $\mathrm{H}(31)$ & $C(64)$ & $C(63)$ & 119(1) & $\mathrm{C}(66)$ & $\mathrm{C}(65)$ & $\mathrm{H}(32)$ & $121(2)$ \\
\hline $\mathrm{H}(32)$ & $C(65)$ & $C(64)$ & $118(2)$ & $C(67)$ & $\mathrm{C}(66)$ & $\mathrm{H}(33)$ & $120(2)$ \\
\hline $\mathrm{H}(33)$ & $C(66)$ & $C(65)$ & $118(2)$ & $\mathrm{C}(68)$ & $\mathrm{C}(67)$ & $\mathrm{H}(34)$ & $120(2)$ \\
\hline $\mathrm{H}(34)$ & $C(67)$ & $C(66)$ & $120(2)$ & $\mathrm{H}(35)$ & $\mathrm{C}(68)$ & $C(63)$ & $118(1)$ \\
\hline $\mathrm{H}(35)$ & $\mathrm{C}(68)$ & $C(67)$ & $119(2)$ & $\mathrm{C}(71)$ & $\mathrm{C}(70)$ & $\mathrm{H}(36)$ & $120(2)$ \\
\hline $\mathrm{H}(36)$ & $C(70)$ & $C(69)$ & 121(3) & $\mathrm{C}(72)$ & $\mathrm{C}(71)$ & $\mathrm{H}(37)$ & $118(4)$ \\
\hline $\mathrm{H}(37)$ & $\mathrm{C}(71)$ & $C(70)$ & $120(4)$ & $\mathrm{C}(73)$ & $\mathrm{C}(72)$ & $\mathrm{H}(38)$ & 121(4) \\
\hline $\mathrm{H}(38)$ & $\mathrm{C}(72)$ & $\mathrm{C}(71)$ & 118(3) & $C(74)$ & $\mathrm{C}(73)$ & $\mathrm{H}(39)$ & $120(4)$ \\
\hline $\mathrm{H}(39)$ & $\mathrm{C}(73)$ & $C(72)$ & 119(4) & $\mathrm{H}(40)$ & $\mathrm{C}(74)$ & C(69) & $119(2)$ \\
\hline $\mathrm{H}(40)$ & $\mathrm{C}(74)$ & $C(73)$ & 118(3) & $C(76)$ & $\mathrm{C}(75)$ & $\mathrm{H}(55)$ & $99(3)$ \\
\hline$C(76)$ & $C(75)$ & $\mathrm{H}(56)$ & $119(3)$ & $\mathrm{H}(55)$ & $\mathrm{C}(75)$ & $H(56)$ & $109(3)$ \\
\hline $\mathrm{H}(55)$ & $C(75)$ & $\mathrm{P}(2)$ & $103(2)$ & $\mathrm{H}(56)$ & $\mathrm{C}(75)$ & $\mathrm{P}(2)$ & $114(2)$ \\
\hline $\mathrm{H}(102)$ & $C(76)$ & $\mathrm{H}(103)$ & $109(5)$ & $\mathrm{H}(102)$ & $\mathrm{C}(76)$ & $\mathrm{H}(104)$ & $109(6)$ \\
\hline $\mathrm{H}(102)$ & $C(76)$ & $C(75)$ & $108(4)$ & $\mathrm{H}(103)$ & $\mathrm{C}(76)$ & $\mathrm{H}(104)$ & $109(5)$ \\
\hline $\mathrm{H}(103)$ & $C(76)$ & $C(75)$ & $99(4)$ & $\mathrm{H}(104)$ & $C(76)$ & $C(75)$ & $119(4)$ \\
\hline $\mathrm{C}(78)$ & $C(77)$ & $\mathrm{H}(51)$ & $105(6)$ & $\mathrm{C}(78)$ & $\mathrm{C}(77)$ & $\mathrm{H}(52)$ & $113(5)$ \\
\hline $\mathrm{H}(51)$ & $C(77)$ & $\mathrm{H}(52)$ & $109(4)$ & $\mathrm{H}(51)$ & $\mathrm{C}(77)$ & $\mathrm{P}(2)$ & $109(4)$ \\
\hline $\mathrm{H}(52)$ & $C(77)$ & $\mathrm{P}(2)$ & 111(5) & $\mathrm{H}(105)$ & $\mathrm{C}(78)$ & $\mathrm{H}(106)$ & $109(5)$ \\
\hline $\mathrm{H}(105)$ & $\mathrm{C}(78)$ & $\mathrm{H}(107)$ & $109(6)$ & $\mathrm{H}(105)$ & $\mathrm{C}(78)$ & $\mathrm{C}(77)$ & $105(7)$ \\
\hline $\mathrm{H}(106)$ & $\mathrm{C}(78)$ & $\mathrm{H}(107)$ & 109(8) & $\mathrm{H}(106)$ & $\mathrm{C}(78)$ & $\mathrm{C}(77)$ & $112(6)$ \\
\hline $\mathrm{H}(107)$ & $\mathrm{C}(78)$ & $C(77)$ & $110(5)$ & $\mathrm{C}(80)$ & $\mathrm{C}(79)$ & $\mathrm{H}(53)$ & $107(10)$ \\
\hline $\mathrm{C}(80)$ & $\mathrm{C}(79)$ & $\mathrm{H}(54)$ & 112(9) & $\mathrm{H}(53)$ & $\mathrm{C}(79)$ & $\mathrm{H}(54)$ & $109(8)$ \\
\hline $\mathrm{H}(53)$ & $C(79)$ & $\mathrm{P}(2)$ & $105(7)$ & $\mathrm{H}(54)$ & $\mathrm{C}(79)$ & $\mathrm{P}(2)$ & $104(7)$ \\
\hline $\mathrm{H}(108)$ & $C(80)$ & $\mathrm{H}(109)$ & $109(11)$ & $\mathrm{H}(108)$ & $\mathrm{C}(80)$ & $\mathrm{H}(110)$ & 109(9) \\
\hline $\mathrm{H}(108)$ & $C(80)$ & $C(79)$ & 111(10) & H(109) & $\mathrm{C}(80)$ & $\mathrm{H}(110)$ & $109(10)$ \\
\hline H(109) & $C(80)$ & $C(79)$ & $110(9)$ & $\mathrm{H}(110)$ & $\mathrm{C}(80)$ & $\mathrm{C}(79)$ & $106(10)$ \\
\hline
\end{tabular}




\section{Crystallographic Results of complex 4}

\section{Data Collection}

\section{Experimental}

A yellow prism crystal of $\mathrm{C}_{46} \mathrm{H}_{68} \mathrm{~N}_{2} \mathrm{Si}_{2} \mathrm{P}_{2} \mathrm{Pt}_{2}$ having approximate dimensions of $0.15 \mathrm{x}$ $0.20 \times 0.10 \mathrm{~mm}$ was mounted on a glass fiber. All measurements were made on a Rigaku Saturn $\mathrm{CCD}$ area detector with graphite monochromated Mo-K $\alpha$ radiation.

Indexing was performed from 720 images that were exposed for 24 seconds. The crystal-to-detector distance was $44.75 \mathrm{~mm}$.

Cell constants and an orientation matrix for data collection corresponded to a $\mathrm{C}$-centered monoclinic cell with dimensions:

$$
\begin{array}{lr}
\mathrm{a}= & 19.222(5) \AA \\
\mathrm{b}= & 11.727(2) \AA
\end{array} \quad \beta=106.471(10)^{\mathrm{O}}
$$

For $\mathrm{Z}=4$ and F.W. $=1157.36$, the calculated density is $1.59 \mathrm{~g} / \mathrm{cm}^{3}$. Based on the systematic absences of:

hkl: $\quad \mathrm{h}+\mathrm{k} \pm 2 \mathrm{n}$

h0l: $\quad 1 \pm 2 n$

packing considerations, a statistical analysis of intensity distribution, and the successful solution and refinement of the structure, the space group was determined to be:

\section{$\mathrm{C} 2 / \mathrm{c}(\# 15)$}

The data were collected at a temperature of $20 \pm 1^{\circ} \mathrm{C}$ to a maximum $2 \theta$ value of $55.0^{\circ}$. A total of 720 oscillation images were collected. A sweep of data was done using $\omega$ scans from -110.0 to $70.0^{\circ}$ in $0.5^{\circ}$ step, at $\chi=45.0^{\circ}$ and $\phi=0.0^{\circ}$. The exposure rate was 24.0 [sec./ $/{ }^{\circ}$ ]. The detector swing angle was $-20.31^{\circ}$. A second sweep was performed using $\omega$ scans from -110.0 to $70.0^{\circ}$ in $0.5^{\circ}$ step, at $\chi=45.0^{\circ}$ and $\phi=90.0^{\circ}$. The exposure rate was $24.0\left[\mathrm{sec} . /{ }^{\circ}\right]$. The detector swing angle was $-20.31^{\circ}$. The crystal-to-detector distance was $44.75 \mathrm{~mm}$. Readout was performed in the $0.070 \mathrm{~mm}$ pixel mode.

Data Reduction

Of the 16582 reflections that were collected, 5445 were unique $\left(R_{i n t}=0.059\right)$; equivalent reflections were merged. Data were collected and processed using CrystalClear (Rigaku). Net intensities and sigmas were derived as follows:

$$
\begin{aligned}
& \mathrm{F}^{2}=\left[\Sigma\left(\mathrm{P}_{\mathrm{i}}-\mathrm{mB}_{\mathrm{ave}}\right)\right] \cdot \mathrm{Lp}^{-1} \\
& \text { where } \mathrm{P}_{\mathrm{i}} \text { is the value in counts of the } \mathrm{i}^{\text {th }} \text { pixel } \\
& m \text { is the number of pixels in the integration area } \\
& B \text { ave is the background average } \\
& \mathrm{Lp} \text { is the Lorentz and polarization factor } \\
& \mathrm{B}_{\mathrm{ave}}=\Sigma\left(\mathrm{B}_{\mathrm{j}}\right) / \mathrm{n} \\
& \text { where } \mathrm{n} \text { is the number of pixels in the background area } \\
& B_{j} \text { is the value of the } j^{\text {th }} \text { pixel in counts } \\
& \sigma^{2}\left(\mathrm{~F}^{2} \mathrm{hkl}\right)=\left[\left(\Sigma \mathrm{P}_{\mathrm{i}}\right)+\mathrm{m}\left(\left(\Sigma\left(\mathrm{B}_{\mathrm{ave}}-\mathrm{B}_{\mathrm{j}}\right)^{2}\right) /(\mathrm{n}-1)\right)\right] \cdot \mathrm{Lp} \cdot \quad \text { errmul }+\left(\text { erradd } \cdot \mathrm{F}^{2}\right)^{2} \\
& \text { where erradd }=0.00 \\
& \text { errmul }=1.00
\end{aligned}
$$


The linear absorption coefficient, $\mu$, for Mo-K $\alpha$ radiation is $59.0 \mathrm{~cm}^{-1}$. An empirical absorption correction was applied which resulted in transmission factors ranging from 0.79 to 1.00. The data were corrected for Lorentz and polarization effects.

Structure Solution and Refinement

The structure was solved by heavy-atom Patterson methods 2 and expanded using Fourier techniques ${ }^{3}$. The non-hydrogen atoms were refined anisotropically. Hydrogen atoms were refined using the riding model. The final cycle of full-matrix least-squares refinement ${ }^{4}$ on $\mathrm{F}$ was based on 3397 observed reflections (I > 3.00 $(\mathrm{I})$ ) and 278 variable parameters and converged (largest parameter shift was 0.01 times its esd) with unweighted and weighted agreement factors of:

$$
\begin{gathered}
\mathrm{R}=\Sigma\|\mathrm{Fo}|-| \mathrm{Fc}\| / \Sigma|\mathrm{Fo}|=0.044 \\
\mathrm{R}_{\mathrm{W}}=\left[\Sigma \mathrm{w}(|\mathrm{Fo}|-|\mathrm{Fc}|)^{2} / \Sigma \mathrm{F} \mathrm{Fo}^{2}\right]^{1 / 2}=0.073
\end{gathered}
$$

The standard deviation of an observation of unit weight ${ }^{5}$ was 0.97 . A Sheldrick weighting scheme was used. Plots of $\Sigma \mathrm{w}(|\mathrm{Fo}|-|\mathrm{Fc}|)^{2}$ versus $|\mathrm{Fo}|$, reflection order in data collection, sin $\bullet \bullet$ and various classes of indices showed no unusual trends. The maximum and minimum peaks on the final difference Fourier map corresponded to 7.40 and $-5.28 \mathrm{e} / \AA^{3}$, respectively.

Neutral atom scattering factors were taken from Cromer and Waber6. Anomalous dispersion effects were included in Fcalc 7 ; the values for $\Delta \mathrm{f}^{\prime}$ and $\Delta \mathrm{f}^{\prime \prime}$ were those of Creagh and McAuley ${ }^{8}$. The values for the mass attenuation coefficients are those of Creagh and Hubbell 9 . All calculations were performed using the CrystalStructure 10,11 crystallographic software package.

\section{References}

(1) CrystalClear: Rigaku Corporation, 1999. CrystalClear Software User's Guide, Molecular Structure Corporation, (c) 2000.J.W.Pflugrath (1999) Acta Cryst. D55, 1718-1725.

(2) PATTY: Beurskens, P.T., Admiraal, G., Beurskens, G., Bosman, W.P., Garcia-Granda, S., Gould, R.O., Smits, J.M.M. and Smykalla, C. (1992). The DIRDIF program system, Technical Report of the Crystallography Laboratory, University of Nijmegen, The Netherlands.

(3) DIRDIF99: Beurskens, P.T., Admiraal, G., Beurskens, G., Bosman, W.P., de Gelder, R., Israel, R. and Smits, J.M.M.(1999). The DIRDIF-99 program system, Technical Report of the Crystallography Laboratory, University of Nijmegen, The Netherlands.

(4) Least Squares function minimized:

$$
\Sigma w\left(\left|\mathrm{~F}_{\mathrm{O}}\right|-\left|\mathrm{F}_{\mathrm{C}}\right|\right)^{2} \quad \text { where } \mathrm{w}=\text { Least Squares weights. }
$$

(5) Standard deviation of an observation of unit weight:

$$
\begin{array}{ll}
{\left[\sum w\left(\left|\mathrm{~F}_{\mathrm{O}}\right|-\left|\mathrm{F}_{\mathrm{C}}\right|\right)^{2} /\left(\mathrm{N}_{\mathrm{O}}-\mathrm{N}_{\mathrm{V}}\right)\right]^{1 / 2}} \\
\text { where: } & \mathrm{N}_{\mathrm{O}}=\text { number of observations } \\
& \mathrm{N}_{\mathrm{V}}=\text { number of variables }
\end{array}
$$

(6) Cromer, D. T. \& Waber, J. T.; "International Tables for X-ray Crystallography", Vol. IV, The Kynoch Press, Birmingham, England, Table 2.2 A (1974).

(7) Ibers, J. A. \& Hamilton, W. C.; Acta Crystallogr., 17, 781 (1964).

(8) Creagh, D. C. \& McAuley, W.J .; "International Tables for Crystallography", Vol C, (A.J.C.

Wilson, ed.), Kluwer Academic Publishers, Boston, Table 4.2.6.8, pages 219-222 (1992).

(9) Creagh, D. C. \& Hubbell, J.H..; "International Tables for Crystallography", Vol C, (A.J.C. Wilson, ed.), Kluwer Academic Publishers, Boston, Table 4.2.4.3, pages 200-206 (1992).

(10) CrystalStructure 3.5.1: Crystal Structure Analysis Package, Rigaku and Rigaku/MSC 
(2000-2003). 9009 New Trails Dr. The Woodlands TX 77381 USA.

(11) CRYSTALS Issue 10: Watkin, D.J., Prout, C.K. Carruthers, J.R. \& Betteridge, P.W.

Chemical Crystallography Laboratory, Oxford, UK. (1996)

\section{EXPERIMENTAL DETAILS}

Empirical Formula

Formula Weight

Crystal Color, Habit

Crystal Dimensions

Crystal System

Lattice Type

Detector Position

Pixel Size

Lattice Parameters

Space Group

$\mathrm{Z}$ value

$D_{\text {calc }}$

$\mathrm{F} 000$

$\mu(\mathrm{MoK} \bullet)$
A. Crystal Data

$\mathrm{C}_{46} \mathrm{H}_{68} \mathrm{~N}_{2} \mathrm{Si}_{2} \mathrm{P}_{2} \mathrm{Pt}_{2}$

1157.36

yellow, prism

$0.15 \times 0.20 \times 0.10 \mathrm{~mm}$

monoclinic

C-centered

$44.75 \mathrm{~mm}$

$0.035 \mathrm{~mm}$

$\mathrm{a}=19.222(5) \AA$

$\mathrm{b}=11.727(2) \AA$

$\mathrm{c}=22.376(5) \AA$

$\beta=106.471(10)^{o}$

$\mathrm{V}=4836.7(20) \AA^{3}$

$\mathrm{C} 2 / \mathrm{c}(\# 15)$

4

$1.589 \mathrm{~g} / \mathrm{cm}^{3}$

2288.00

$59.02 \mathrm{~cm}^{-1}$

B. Intensity Measurements

Detector

Goniometer

Radiation

Detector Aperture

Data Images

$\omega$ oscillation Range $(\bullet=45.0, \bullet=0.0)$

Exposure Rate

Detector Swing Angle

$\omega$ oscillation Range $(\bullet=45.0, \bullet=90.0)$

Exposure Rate

Detector Swing Angle

Detector Position

Pixel Size

$2 \theta_{\text {max }}$

No. of Reflections Measured
Rigaku Saturn

Rigaku AFC10

$\operatorname{MoK} \alpha(\lambda=0.71070 \AA)$

graphite monochromated

$70 \mathrm{~mm} \times 70 \mathrm{~mm}$

720 exposures

$-110.0-70.0^{\circ}$

$24.0 \mathrm{sec} . / \mathrm{O}$

$-20.31^{\mathrm{O}}$

$-110.0-70.0^{\circ}$

$24.0 \mathrm{sec} . / \mathrm{O}$

$-20.31^{\mathrm{O}}$

$44.75 \mathrm{~mm}$

$0.035 \mathrm{~mm}$

$55.0^{\circ}$

Total: 16582

Unique: $5445\left(\mathrm{R}_{\text {int }}=0.059\right)$ 
Corrections

Lorentz-polarization

Absorption

(trans. factors: $0.7889-1.0000$ )

Structure Solution

Refinement

Function Minimized

Least Squares Weights

Anomalous Dispersion

No. Observations $(\mathrm{I}>3.00 \sigma(\mathrm{I}))$

No. Variables

Reflection/Parameter Ratio

Residuals: R (I>3.00 $\sigma(\mathrm{I}))$

Residuals: Rw (I>3.00б(I))

Goodness of Fit Indicator

Max Shift/Error in Final Cycle

Maximum peak in Final Diff. Map

Minimum peak in Final Diff. Map
C. Structure Solution and Refinement

Patterson Methods (DIRDIF99 PATTY)

Full-matrix least-squares on $\mathrm{F}$

$\Sigma \mathrm{w}(|\mathrm{Fo}|-|\mathrm{Fc}|)^{2}$

$1 /\left[0.0010 \mathrm{Fo}^{2}+2.0000 \bullet\left(\mathrm{Fo}^{2}\right)+0.1000\right]$

All non-hydrogen atoms

3397

278

12.22

0.044

0.073

0.966

0.007

$7.40 \mathrm{e} / \AA^{3}$

$-5.28 \mathrm{e} / \AA^{3}$

Table 1. Atomic coordinates and $\mathrm{B}_{\mathrm{iso}} / \mathrm{B}_{\mathrm{eq}}$

$\begin{array}{lcccc}\text { atom } & \mathrm{x} & \mathrm{y} & \mathrm{z} & \mathrm{B} \text { eq } \\ \mathrm{Pt}(1) & 0.1034 & -0.0084 & 0.2944 & 1.130 \\ \mathrm{P}(1) & 0.1694 & -0.0347 & 0.3980 & 1.448 \\ \mathrm{Si}(1) & 0.0158 & 0.0078 & 0.1951 & 1.148 \\ \mathrm{~N}(1) & 0.2271 & -0.0719 & 0.2327 & 1.729 \\ \mathrm{C}(1) & 0.0346 & 0.1458 & 0.1593 & 1.372 \\ \mathrm{C}(2) & 0.0865 & 0.1584 & 0.1276 & 1.406 \\ \mathrm{C}(3) & 0.1002 & 0.2594 & 0.1015 & 2.073 \\ \mathrm{C}(4) & 0.0569 & 0.3555 & 0.1041 & 2.060 \\ \mathrm{C}(5) & 0.0049 & 0.3473 & 0.1354 & 2.288 \\ \mathrm{C}(6) & -0.0081 & 0.2448 & 0.1620 & 1.848 \\ \mathrm{C}(7) & 0.0295 & -0.1131 & 0.1435 & 0.906 \\ \mathrm{C}(8) & 0.0425 & -0.2234 & 0.1695 & 1.867 \\ \mathrm{C}(9) & 0.0496 & -0.3181 & 0.1323 & 2.532 \\ \mathrm{C}(10) & 0.0457 & -0.3041 & 0.0731 & 2.543 \\ \mathrm{C}(11) & 0.0328 & -0.1973 & 0.0447 & 1.719 \\ \mathrm{C}(12) & 0.0250 & -0.1030 & 0.0783 & 1.812 \\ \mathrm{C}(13) & 0.1489 & 0.0488 & 0.4609 & 1.798 \\ \mathrm{C}(14) & 0.1682 & 0.1768 & 0.4575 & 2.535 \\ \mathrm{C}(15) & 0.2675 & -0.0132 & 0.4141 & 1.676 \\ \mathrm{C}(16) & 0.3132 & -0.0280 & 0.4806 & 2.618 \\ \mathrm{C}(17) & 0.1627 & -0.1810 & 0.4240 & 2.437\end{array}$




\begin{tabular}{|c|c|c|c|c|}
\hline $\mathrm{C}(18)$ & 0.1724 & -0.2702 & 0.3790 & 2.617 \\
\hline$C(19)$ & 0.1819 & -0.0442 & 0.2571 & 1.671 \\
\hline$C(20)$ & 0.2746 & -0.1206 & 0.2004 & 2.013 \\
\hline $\mathrm{C}(21)$ & 0.3456 & -0.0595 & 0.2240 & 3.486 \\
\hline$C(22)$ & 0.2824 & -0.2448 & 0.2164 & 3.353 \\
\hline $\mathrm{C}(23)$ & 0.2410 & -0.0987 & 0.1307 & 2.864 \\
\hline $\mathrm{H}(1)$ & 0.1156 & 0.0941 & 0.1248 & 1.773 \\
\hline $\mathrm{H}(2)$ & 0.1375 & 0.2647 & 0.0814 & 2.628 \\
\hline $\mathrm{H}(3)$ & 0.0629 & 0.4243 & 0.0837 & 2.559 \\
\hline $\mathrm{H}(4)$ & -0.0211 & 0.4136 & 0.1407 & 2.875 \\
\hline $\mathrm{H}(5)$ & -0.0469 & 0.2397 & 0.1804 & 2.422 \\
\hline $\mathrm{H}(6)$ & 0.0478 & -0.2335 & 0.2127 & 2.314 \\
\hline $\mathrm{H}(7)$ & 0.0585 & -0.3919 & 0.1503 & 2.873 \\
\hline $\mathrm{H}(8)$ & 0.0499 & -0.3682 & 0.0484 & 3.140 \\
\hline $\mathrm{H}(9)$ & 0.0285 & -0.1891 & 0.0015 & 2.153 \\
\hline $\mathrm{H}(10)$ & 0.0188 & -0.0297 & 0.0595 & 2.185 \\
\hline $\mathrm{H}(11)$ & 0.0985 & 0.0430 & 0.4566 & 2.148 \\
\hline $\mathrm{H}(12)$ & 0.1755 & 0.0184 & 0.5001 & 2.146 \\
\hline $\mathrm{H}(13)$ & 0.2187 & 0.1836 & 0.4621 & 3.185 \\
\hline $\mathrm{H}(14)$ & 0.1417 & 0.2082 & 0.4186 & 3.185 \\
\hline $\mathrm{H}(15)$ & 0.1566 & 0.2167 & 0.4903 & 3.183 \\
\hline $\mathrm{H}(16)$ & 0.2845 & -0.0653 & 0.3890 & 1.897 \\
\hline $\mathrm{H}(17)$ & 0.2748 & 0.0626 & 0.4022 & 1.897 \\
\hline $\mathrm{H}(18)$ & 0.3079 & -0.1040 & 0.4933 & 3.184 \\
\hline $\mathrm{H}(19)$ & 0.3627 & -0.0138 & 0.4836 & 3.186 \\
\hline $\mathrm{H}(20)$ & 0.2974 & 0.0236 & 0.5068 & 3.184 \\
\hline $\mathrm{H}(21)$ & 0.1991 & -0.1918 & 0.4624 & 3.199 \\
\hline $\mathrm{H}(22)$ & 0.1162 & -0.1910 & 0.4302 & 3.199 \\
\hline $\mathrm{H}(23)$ & 0.1679 & -0.3441 & 0.3949 & 3.051 \\
\hline $\mathrm{H}(24)$ & 0.1364 & -0.2603 & 0.3403 & 3.051 \\
\hline $\mathrm{H}(25)$ & 0.2191 & -0.2622 & 0.3730 & 3.049 \\
\hline $\mathrm{H}(26)$ & 0.3648 & -0.0700 & 0.2677 & 4.148 \\
\hline $\mathrm{H}(27)$ & 0.3786 & -0.0895 & 0.2035 & 4.148 \\
\hline $\mathrm{H}(28)$ & 0.3386 & 0.0195 & 0.2151 & 4.148 \\
\hline $\mathrm{H}(29)$ & 0.2359 & -0.2797 & 0.2034 & 4.156 \\
\hline $\mathrm{H}(30)$ & 0.3137 & -0.2801 & 0.1959 & 4.153 \\
\hline $\mathrm{H}(31)$ & 0.3020 & -0.2534 & 0.2602 & 4.155 \\
\hline $\mathrm{H}(32)$ & 0.2354 & -0.0190 & 0.1231 & 3.526 \\
\hline $\mathrm{H}(33)$ & 0.2712 & -0.1296 & 0.1078 & 3.525 \\
\hline $\mathrm{H}(34)$ & 0.1948 & -0.1344 & 0.1181 & 3.527 \\
\hline
\end{tabular}

$\mathrm{B}_{\mathrm{eq}}=8 / 3 \pi^{2}\left(\mathrm{U}_{11}\left(\mathrm{aa}^{*}\right)^{2}+\mathrm{U}_{22}\left(\mathrm{bb}^{*}\right)^{2}+\mathrm{U}_{33}(\mathrm{cc} *)^{2}+2 \mathrm{U}_{12}(\mathrm{aa} * \mathrm{bb} *) \cos \gamma+2 \mathrm{U}_{13}\left(\mathrm{aa}^{*} \mathrm{cc} *\right) \cos \beta+\right.$ $\left.2 \mathrm{U}_{23}(\mathrm{bb} * \mathrm{cc} *) \cos \alpha\right)$ ] 
Table 2. Anisotropic Displacement Parameters

\begin{tabular}{|c|c|c|c|c|c|c|}
\hline atom & $\mathrm{U}_{11}$ & $\mathrm{U}_{22}$ & U33 & $\mathrm{U}_{12}$ & $\mathrm{U}_{13}$ & $\mathrm{U}_{23}$ \\
\hline $\operatorname{Pt}(1)$ & 0.0185 & 0.0137 & 0.0124 & 0.0029 & 0.0072 & -0.0002 \\
\hline $\mathrm{P}(1)$ & 0.0201 & 0.0206 & 0.0161 & 0.0029 & 0.0080 & 0.0017 \\
\hline $\mathrm{Si}(1)$ & 0.0197 & 0.0134 & 0.0117 & 0.0064 & 0.0064 & 0.0032 \\
\hline $\mathrm{N}(1)$ & 0.0295 & 0.0249 & 0.0133 & 0.0032 & 0.0092 & -0.0008 \\
\hline$C(1)$ & 0.0137 & 0.0181 & 0.0108 & -0.0067 & -0.0119 & -0.0078 \\
\hline$C(2)$ & 0.0134 & 0.0238 & 0.0190 & 0.0052 & 0.0092 & 0.0091 \\
\hline$C(3)$ & 0.0194 & 0.0257 & 0.0381 & -0.0090 & 0.0155 & 0.0021 \\
\hline$C(4)$ & 0.0501 & 0.0182 & 0.0127 & -0.0067 & 0.0133 & 0.0029 \\
\hline$C(5)$ & 0.0304 & 0.0121 & 0.0484 & 0.0010 & 0.0177 & -0.0032 \\
\hline$C(6)$ & 0.0261 & 0.0163 & 0.0342 & -0.0036 & 0.0190 & -0.0046 \\
\hline$C(7)$ & 0.0063 & 0.0206 & -0.0005 & 0.0022 & -0.0120 & -0.0028 \\
\hline$C(8)$ & 0.0284 & 0.0248 & 0.0200 & 0.0029 & 0.0106 & 0.0023 \\
\hline$C(9)$ & 0.0478 & 0.0204 & 0.0228 & -0.0015 & 0.0015 & -0.0060 \\
\hline$C(10)$ & 0.0464 & 0.0185 & 0.0345 & 0.0100 & 0.0160 & 0.0033 \\
\hline $\mathrm{C}(11)$ & 0.0235 & 0.0290 & 0.0157 & -0.0076 & 0.0103 & -0.0065 \\
\hline$C(12)$ & 0.0239 & 0.0168 & 0.0285 & -0.0029 & 0.0080 & -0.0024 \\
\hline$C(13)$ & 0.0121 & 0.0351 & 0.0208 & -0.0069 & 0.0041 & -0.0103 \\
\hline $\mathrm{C}(14)$ & 0.0251 & 0.0338 & 0.0420 & -0.0075 & 0.0169 & -0.0137 \\
\hline$C(15)$ & 0.0141 & 0.0335 & 0.0124 & -0.0093 & -0.0021 & -0.0129 \\
\hline$C(16)$ & 0.0127 & 0.0496 & 0.0385 & 0.0129 & 0.0095 & 0.0034 \\
\hline $\mathrm{C}(17)$ & 0.0413 & 0.0309 & 0.0291 & 0.0200 & 0.0240 & 0.0200 \\
\hline $\mathrm{C}(18)$ & 0.0432 & 0.0300 & 0.0233 & 0.0053 & 0.0048 & -0.0052 \\
\hline C(19) & 0.0416 & 0.0206 & 0.0075 & 0.0029 & 0.0170 & 0.0003 \\
\hline C(20) & 0.0204 & 0.0366 & 0.0209 & 0.0087 & 0.0082 & -0.0001 \\
\hline $\mathrm{C}(21)$ & 0.0146 & 0.0733 & 0.0434 & 0.0101 & 0.0063 & -0.0015 \\
\hline $\mathrm{C}(22)$ & 0.0678 & 0.0432 & 0.0205 & 0.0257 & 0.0193 & 0.0040 \\
\hline $\mathrm{C}(23)$ & 0.0368 & 0.0561 & 0.0188 & 0.0136 & 0.0125 & -0.0094 \\
\hline
\end{tabular}

Table 3. Bond lengths ( $\mathrm{A})$

$\begin{array}{llllll}\text { atom } & \text { atom } & \text { distance } & \text { atom } & \text { atom } & \text { distance } \\ \operatorname{Pt}(1) & \operatorname{Pt}(1)^{*} & 3.9067(7) & \operatorname{Pt}(1) & \mathrm{P}(1) & 2.327(3) \\ \operatorname{Pt}(1) & \mathrm{Si}(1) & 2.385(3) & \mathrm{Pt}(1) & \mathrm{Si}(1)^{*} & 2.376(3) \\ \mathrm{Pt}(1) & \mathrm{C}(19) & 1.96(2) & \mathrm{Si}(1) & \mathrm{Si}(1)^{*} & 2.695(5) \\ \mathrm{P}(1) & \mathrm{C}(13) & 1.85(2) & \mathrm{P}(1) & \mathrm{C}(15) & 1.83(1) \\ \mathrm{P}(1) & \mathrm{C}(17) & 1.83(2) & \mathrm{Si}(1) & \mathrm{C}(1) & 1.89(1) \\ \mathrm{Si}(1) & \mathrm{C}(7) & 1.89(1) & \mathrm{N}(1) & \mathrm{C}(19) & 1.20(2) \\ \mathrm{N}(1) & \mathrm{C}(20) & 1.43(2) & \mathrm{C}(1) & \mathrm{C}(2) & 1.39(2) \\ \mathrm{C}(1) & \mathrm{C}(6) & 1.43(2) & \mathrm{C}(2) & \mathrm{C}(3) & 1.38(2) \\ \mathrm{C}(3) & \mathrm{C}(4) & 1.41(2) & \mathrm{C}(4) & \mathrm{C}(5) & 1.38(3) \\ \mathrm{C}(5) & \mathrm{C}(6) & 1.39(2) & \mathrm{C}(7) & \mathrm{C}(8) & 1.41(2)\end{array}$




$\begin{array}{llllll}\mathrm{C}(7) & \mathrm{C}(12) & 1.44(2) & \mathrm{C}(8) & \mathrm{C}(9) & 1.42(2) \\ \mathrm{C}(9) & \mathrm{C}(10) & 1.32(3) & \mathrm{C}(10) & \mathrm{C}(11) & 1.39(2) \\ \mathrm{C}(11) & \mathrm{C}(12) & 1.37(2) & \mathrm{C}(13) & \mathrm{C}(14) & 1.55(2) \\ \mathrm{C}(15) & \mathrm{C}(16) & 1.51(2) & \mathrm{C}(17) & \mathrm{C}(18) & 1.50(2) \\ \mathrm{C}(20) & \mathrm{C}(21) & 1.50(2) & \mathrm{C}(20) & \mathrm{C}(22) & 1.50(2) \\ \mathrm{C}(20) & \mathrm{C}(23) & 1.53(2) & & & \\ & & & & \\ \text { Table 4. Bond lengths involving hydrogens }(\AA) & & & \\ \text { atom } & \text { atom } & \text { distance } & \text { atom } & \text { atom } & \text { distance } \\ \mathrm{C}(2) & \mathrm{H}(1) & 0.95(2) & \mathrm{C}(3) & \mathrm{H}(2) & 0.95(3) \\ \mathrm{C}(4) & \mathrm{H}(3) & 0.95(2) & \mathrm{C}(5) & \mathrm{H}(4) & 0.95(2) \\ \mathrm{C}(6) & \mathrm{H}(5) & 0.95(3) & \mathrm{C}(8) & \mathrm{H}(6) & 0.95(2) \\ \mathrm{C}(9) & \mathrm{H}(7) & 0.95(2) & \mathrm{C}(10) & \mathrm{H}(8) & 0.95(2) \\ \mathrm{C}(11) & \mathrm{H}(9) & 0.95(2) & \mathrm{C}(12) & \mathrm{H}(10) & 0.95(2) \\ \mathrm{C}(13) & \mathrm{H}(11) & 0.95(2) & \mathrm{C}(13) & \mathrm{H}(12) & 0.95(2) \\ \mathrm{C}(14) & \mathrm{H}(13) & 0.95(2) & \mathrm{C}(14) & \mathrm{H}(14) & 0.95(2) \\ \mathrm{C}(14) & \mathrm{H}(15) & 0.95(3) & \mathrm{C}(15) & \mathrm{H}(16) & 0.95(2) \\ \mathrm{C}(15) & \mathrm{H}(17) & 0.95(2) & \mathrm{C}(16) & \mathrm{H}(18) & 0.95(2) \\ \mathrm{C}(16) & \mathrm{H}(19) & 0.95(2) & \mathrm{C}(16) & \mathrm{H}(20) & 0.95(2) \\ \mathrm{C}(17) & \mathrm{H}(21) & 0.95(2) & \mathrm{C}(17) & \mathrm{H}(22) & 0.95(3) \\ \mathrm{C}(18) & \mathrm{H}(23) & 0.95(2) & \mathrm{C}(18) & \mathrm{H}(24) & 0.95(2) \\ \mathrm{C}(18) & \mathrm{H}(25) & 0.95(3) & \mathrm{C}(21) & \mathrm{H}(26) & 0.95(3) \\ \mathrm{C}(21) & \mathrm{H}(27) & 0.95(3) & \mathrm{C}(21) & \mathrm{H}(28) & 0.95(3) \\ \mathrm{C}(22) & \mathrm{H}(29) & 0.95(3) & \mathrm{C}(22) & \mathrm{H}(30) & 0.95(3) \\ \mathrm{C}(22) & \mathrm{H}(31) & 0.95(2) & \mathrm{C}(23) & \mathrm{H}(32) & 0.95(2) \\ \mathrm{C}(23) & \mathrm{H}(33) & 0.95(3) & \mathrm{C}(23) & \mathrm{H}(34) & 0.95(2)\end{array}$

Table 5. Bond angles (O)

$\begin{array}{llllllll}\text { atom } & \text { atom } & \text { atom } & \text { angle } & \text { atom } & \text { atom } & \text { atom } & \text { angle } \\ \mathrm{P}(1) & \mathrm{Pt}(1) & \mathrm{Si}(1) & 168.7(1) & \mathrm{P}(1) & \mathrm{Pt}(1) & \mathrm{C}(19) & 97.3(4) \\ \mathrm{P}(1) & \mathrm{Pt}(1) & \mathrm{Si}(1)^{*} & 100.4(1) & \mathrm{Si}(1) & \mathrm{Pt}(1) & \mathrm{Si}(1)^{*} & 68.94(9) \\ \mathrm{Si}(1) & \mathrm{Pt}(1) & \mathrm{C}(19) & 92.6(4) & \mathrm{C}(19) & \mathrm{Pt}(1) & \mathrm{Si}(1)^{*} & 159.6(4) \\ \mathrm{C}(13) & \mathrm{P}(1) & \mathrm{C}(15) & 101.9(6) & \mathrm{C}(13) & \mathrm{P}(1) & \mathrm{C}(17) & 101.9(8) \\ \mathrm{C}(13) & \mathrm{P}(1) & \mathrm{Pt}(1) & 121.1(4) & \mathrm{C}(15) & \mathrm{P}(1) & \mathrm{C}(17) & 103.2(7) \\ \mathrm{C}(15) & \mathrm{P}(1) & \mathrm{Pt}(1) & 114.6(4) & \mathrm{C}(17) & \mathrm{P}(1) & \mathrm{Pt}(1) & 112.0(5) \\ \mathrm{C}(1) & \mathrm{Si}(1) & \mathrm{C}(7) & 107.9(6) & \mathrm{C}(1) & \mathrm{Si}(1) & \mathrm{Pt}(1) & 107.1(4) \\ \mathrm{C}(1) & \mathrm{Si}(1) & \mathrm{Pt}(1)^{*} & 114.6(5) & \mathrm{C}(7) & \mathrm{Si}(1) & \mathrm{Pt}(1) & 109.0(4) \\ \mathrm{C}(7) & \mathrm{Si}(1) & \mathrm{Pt}(1)^{*} & 107.8(4) & \mathrm{Pt}(1) & \mathrm{Si}(1) & \mathrm{Pt}(1)^{*} & 110.3(1) \\ \mathrm{C}(19) & \mathrm{N}(1) & \mathrm{C}(20) & 171(1) & \mathrm{C}(2) & \mathrm{C}(1) & \mathrm{C}(6) & 116(1) \\ \mathrm{C}(2) & \mathrm{C}(1) & \mathrm{Si}(1) & 124(1) & \mathrm{C}(6) & \mathrm{C}(1) & \mathrm{Si}(1) & 119(1) \\ \mathrm{C}(3) & \mathrm{C}(2) & \mathrm{C}(1) & 124(1) & \mathrm{C}(4) & \mathrm{C}(3) & \mathrm{C}(2) & 118(1) \\ \mathrm{C}(5) & \mathrm{C}(4) & \mathrm{C}(3) & 119(1) & \mathrm{C}(6) & \mathrm{C}(5) & \mathrm{C}(4) & 121(1) \\ \mathrm{C}(1) & \mathrm{C}(6) & \mathrm{C}(5) & 120(1) & \mathrm{C}(8) & \mathrm{C}(7) & \mathrm{C}(12) & 116(1)\end{array}$




$\begin{array}{llllllll}\mathrm{C}(8) & \mathrm{C}(7) & \mathrm{Si}(1) & 118(1) & \mathrm{C}(12) & \mathrm{C}(7) & \mathrm{Si}(1) & 125.2(9) \\ \mathrm{C}(9) & \mathrm{C}(8) & \mathrm{C}(7) & 120(1) & \mathrm{C}(10) & \mathrm{C}(9) & \mathrm{C}(8) & 120(1) \\ \mathrm{C}(11) & \mathrm{C}(10) & \mathrm{C}(9) & 121(1) & \mathrm{C}(12) & \mathrm{C}(11) & \mathrm{C}(10) & 120(1) \\ \mathrm{C}(7) & \mathrm{C}(12) & \mathrm{C}(11) & 120(1) & \mathrm{C}(14) & \mathrm{C}(13) & \mathrm{P}(1) & 111(1) \\ \mathrm{C}(16) & \mathrm{C}(15) & \mathrm{P}(1) & 117(1) & \mathrm{C}(18) & \mathrm{C}(17) & \mathrm{P}(1) & 114(1) \\ \mathrm{Pt}(1) & \mathrm{C}(19) & \mathrm{N}(1) & 175(1) & \mathrm{C}(21) & \mathrm{C}(20) & \mathrm{C}(22) & 111(1) \\ \mathrm{C}(21) & \mathrm{C}(20) & \mathrm{C}(23) & 111(1) & \mathrm{C}(21) & \mathrm{C}(20) & \mathrm{N}(1) & 106(1) \\ \mathrm{C}(22) & \mathrm{C}(20) & \mathrm{C}(23) & 113(1) & \mathrm{C}(22) & \mathrm{C}(20) & \mathrm{N}(1) & 107(1) \\ \mathrm{C}(23) & \mathrm{C}(20) & \mathrm{N}(1) & 107(1) & & & & \end{array}$

Table 6. Bond angles involving hydrogens (O)

\begin{tabular}{|c|c|c|c|c|c|c|c|}
\hline atom & atom & atom & angle & atom & atom & atom & angle \\
\hline $\mathrm{C}(3)$ & $\mathrm{C}(2)$ & $\mathrm{H}(1)$ & $117(1)$ & $\mathrm{H}(1)$ & $\mathrm{C}(2)$ & $\mathrm{C}(1)$ & $118(1)$ \\
\hline C(4) & $\mathrm{C}(3)$ & $\mathrm{H}(2)$ & $120(1)$ & $\mathrm{H}(2)$ & $\mathrm{C}(3)$ & $\mathrm{C}(2)$ & 121(1) \\
\hline $\mathrm{C}(5)$ & $\mathrm{C}(4)$ & $\mathrm{H}(3)$ & $120(1)$ & $\mathrm{H}(3)$ & $\mathrm{C}(4)$ & $\mathrm{C}(3)$ & $120(2)$ \\
\hline$C(6)$ & $\mathrm{C}(5)$ & $\mathrm{H}(4)$ & $119(2)$ & $\mathrm{H}(4)$ & $C(5)$ & $C(4)$ & 119(1) \\
\hline $\mathrm{H}(5)$ & $\mathrm{C}(6)$ & $\mathrm{C}(1)$ & 119(1) & $\mathrm{H}(5)$ & $\mathrm{C}(6)$ & $\mathrm{C}(5)$ & 119(1) \\
\hline $\mathrm{C}(9)$ & $\mathrm{C}(8)$ & $\mathrm{H}(6)$ & $120(1)$ & $\mathrm{H}(6)$ & $\mathrm{C}(8)$ & $C(7)$ & 119(1) \\
\hline$C(10)$ & $\mathrm{C}(9)$ & $\mathrm{H}(7)$ & 119(1) & $\mathrm{H}(7)$ & $\mathrm{C}(9)$ & $\mathrm{C}(8)$ & $119(2)$ \\
\hline$C(11)$ & $\mathrm{C}(10)$ & $\mathrm{H}(8)$ & $118(2)$ & $\mathrm{H}(8)$ & $\mathrm{C}(10)$ & $\mathrm{C}(9)$ & $119(1)$ \\
\hline$C(12)$ & $\mathrm{C}(11)$ & $\mathrm{H}(9)$ & 119(1) & $\mathrm{H}(9)$ & $\mathrm{C}(11)$ & $C(10)$ & $120(1)$ \\
\hline $\mathrm{H}(10)$ & $\mathrm{C}(12)$ & $C(7)$ & 119(1) & $\mathrm{H}(10)$ & $\mathrm{C}(12)$ & $\mathrm{C}(11)$ & $120(1)$ \\
\hline$C(14)$ & $\mathrm{C}(13)$ & $\mathrm{H}(11)$ & $108(1)$ & $C(14)$ & $\mathrm{C}(13)$ & $\mathrm{H}(12)$ & $109(1)$ \\
\hline $\mathrm{H}(11)$ & $\mathrm{C}(13)$ & $\mathrm{H}(12)$ & $109(2)$ & $\mathrm{H}(11)$ & $\mathrm{C}(13)$ & $\mathrm{P}(1)$ & $108(1)$ \\
\hline $\mathrm{H}(12)$ & $\mathrm{C}(13)$ & $\mathrm{P}(1)$ & $109(1)$ & $\mathrm{H}(13)$ & $C(14)$ & $\mathrm{H}(14)$ & $109(2)$ \\
\hline $\mathrm{H}(13)$ & $\mathrm{C}(14)$ & $\mathrm{H}(15)$ & $109(2)$ & $\mathrm{H}(13)$ & $\mathrm{C}(14)$ & $C(13)$ & 109(1) \\
\hline $\mathrm{H}(14)$ & $\mathrm{C}(14)$ & $\mathrm{H}(15)$ & $109(2)$ & $\mathrm{H}(14)$ & $\mathrm{C}(14)$ & $\mathrm{C}(13)$ & $110(1)$ \\
\hline $\mathrm{H}(15)$ & $\mathrm{C}(14)$ & $\mathrm{C}(13)$ & $109(2)$ & $C(16)$ & $C(15)$ & $\mathrm{H}(16)$ & $107(1)$ \\
\hline$C(16)$ & $C(15)$ & $\mathrm{H}(17)$ & 107(1) & $\mathrm{H}(16)$ & $C(15)$ & $\mathrm{H}(17)$ & 109(1) \\
\hline $\mathrm{H}(16)$ & $\mathrm{C}(15)$ & $\mathrm{P}(1)$ & 107(1) & $\mathrm{H}(17)$ & $C(15)$ & $\mathrm{P}(1)$ & $107(1)$ \\
\hline $\mathrm{H}(18)$ & $C(16)$ & $\mathrm{H}(19)$ & $109(2)$ & $\mathrm{H}(18)$ & $C(16)$ & $\mathrm{H}(20)$ & $109(2)$ \\
\hline $\mathrm{H}(18)$ & $C(16)$ & $C(15)$ & $108(1)$ & $\mathrm{H}(19)$ & $C(16)$ & $\mathrm{H}(20)$ & $109(2)$ \\
\hline $\mathrm{H}(19)$ & $\mathrm{C}(16)$ & $C(15)$ & 109(1) & $\mathrm{H}(20)$ & $C(16)$ & $C(15)$ & 109(1) \\
\hline $\mathrm{C}(18)$ & $\mathrm{C}(17)$ & $\mathrm{H}(21)$ & $108(1)$ & $\mathrm{C}(18)$ & $C(17)$ & $\mathrm{H}(22)$ & $108(1)$ \\
\hline $\mathrm{H}(21)$ & $\mathrm{C}(17)$ & $\mathrm{H}(22)$ & $109(2)$ & $\mathrm{H}(21)$ & $C(17)$ & $\mathrm{P}(1)$ & $108(1)$ \\
\hline $\mathrm{H}(22)$ & $\mathrm{C}(17)$ & $\mathrm{P}(1)$ & $108(1)$ & $\mathrm{H}(23)$ & $\mathrm{C}(18)$ & $\mathrm{H}(24)$ & $109(2)$ \\
\hline $\mathrm{H}(23)$ & $\mathrm{C}(18)$ & $\mathrm{H}(25)$ & $109(2)$ & $\mathrm{H}(23)$ & $\mathrm{C}(18)$ & $C(17)$ & $110(2)$ \\
\hline $\mathrm{H}(24)$ & $\mathrm{C}(18)$ & $\mathrm{H}(25)$ & $109(2)$ & $\mathrm{H}(24)$ & $\mathrm{C}(18)$ & $C(17)$ & 109(1) \\
\hline $\mathrm{H}(25)$ & $\mathrm{C}(18)$ & $\mathrm{C}(17)$ & 109(1) & $\mathrm{H}(26)$ & $\mathrm{C}(21)$ & $\mathrm{H}(27)$ & $109(2)$ \\
\hline $\mathrm{H}(26)$ & $\mathrm{C}(21)$ & $\mathrm{H}(28)$ & $109(2)$ & $\mathrm{H}(26)$ & $\mathrm{C}(21)$ & $C(20)$ & $110(2)$ \\
\hline $\mathrm{H}(27)$ & $\mathrm{C}(21)$ & $\mathrm{H}(28)$ & $109(2)$ & $\mathrm{H}(27)$ & $\mathrm{C}(21)$ & $C(20)$ & $108(2)$ \\
\hline $\mathrm{H}(28)$ & $\mathrm{C}(21)$ & $\mathrm{C}(20)$ & $109(1)$ & $\mathrm{H}(29)$ & $C(22)$ & $\mathrm{H}(30)$ & $109(2)$ \\
\hline $\mathrm{H}(29)$ & $\mathrm{C}(22)$ & $\mathrm{H}(31)$ & $109(2)$ & $\mathrm{H}(29)$ & $\mathrm{C}(22)$ & $C(20)$ & $108(1)$ \\
\hline
\end{tabular}




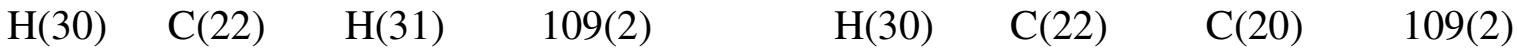



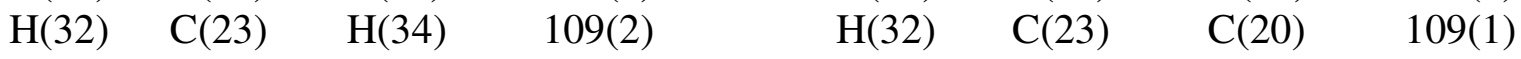

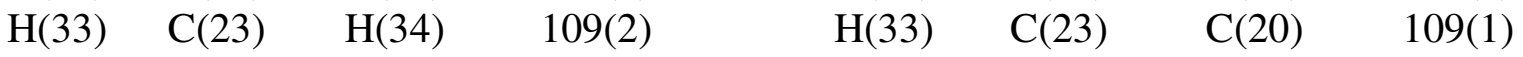

$\mathrm{H}(34) \quad \mathrm{C}(23) \quad \mathrm{C}(20) \quad 108(1)$ 